\title{
22. MINERALOGY AND MINERALOGIC TRENDS IN SEDIMENTS FROM THE TASMAN AND CORAL SEAS, LEG 21 DEEP SEA DRILLING PROJECT ${ }^{1}$
}

\author{
J. C. Matti, Ivar Zemmels, and Harry E. Cook, Department of Geology, \\ University of California, Riverside, California
}

\section{INTRODUCTION}

This report synthesizes X-ray mineralogy data from Sites 203, 204, 205, 206, 207, 208, 209, and 210 on Leg 21 in the Tasman and Coral seas. The discussion attempts to characterize the mineralogy of the various sedimentary horizons insofar as sample material permits. Because of intermittent coring and the limited number of samples submitted to the University of California, Riverside X-ray facility, it is not certain whether these results are representative of the sedimentary section at the sites drilled. Hence, our conclusions must be viewed as tentative.

The results and discussion portion of this paper is a descriptive treatment of the X-ray mineralogy data from each site: (1) the $\mathrm{X}$-ray mineralogy at each site is summarized: (2) general mineralogic trends are outlined, and mineralogic changes associated with lithologic unit boundaries and unconformities are discussed; (3) specific observations are made regarding sediment textures, geologically unique mineral occurrences, and unique mineral associations; and (4) mineralogic correlations are attempted between different sites.

\section{METHODS}

Semiquantitative determinations of the mineral composition of bulk samples, $2-20 \mu$ and $<2 \mu$ fractions were performed according to the methods described in the reports of Legs 1 and 2 and in Appendix III of Volume IV. The mineral analyses of the $2-20 \mu$ and $<2 \mu$ fractions were performed on carbonate-free residues.

The X-ray mineralogy results of this study are summarized in Tables 1 to 4 . The mineralogy data are presented in Tables 5 to 14 and Figures 1 to 30 . Sediment ages, lithologic units, and compositional nomenclature of the sediment types in Figures 1 to 30 and Tables 1 to 4 are from the DSDP Leg 21 Hole Summaries and a subsequent update supplied by Dr. T.A. Davies, DSDP. The stratigraphic positions of samples submitted for X-ray diffraction analysis from Leg 21 are listed in Tables 1 to 4 . The sample depth (in meters) below the sea floor in Tables 1 to 4 identifies the samples as they are reported in Tables 5 to 14 .

Several unidentified minerals were detected in Leg 21 samples. Their abundances were determined on a semiquantitative basis using a hypothetical mineral concentration factor of 3.0. Unidentified minerals are reported on a ranked, semiqualitative scale, as outlined below:

\footnotetext{
${ }^{1}$ Institute of Geophysics and Planetary Physics, University of California, Riverside, Contribution No. 73-24.
}

Trace: (5\%); diffraction pattern was weak and identification was made on the basis of two major diagnostic peaks.

Present: (5\%-25\%); a number of peaks of the mineral are visible in the diffraction pattern.

Abundant: (25\%-65\%); diffraction peaks of the mineral are prominent in the total diffraction pattern, but the peaks of other minerals are of an equivalent intensity.

Major: (65\%); the diffraction peaks of the mineral dominate the diffraction pattern.

Although a certain quantity of the unidentified minerals is implied, their concentration is not included in the concentrations of identified minerals, which are summed to $100 \%$.

The terms "Trace," "Present," etc. are also used in discussing identified minerals in the text of this report and imply the concentration ranges as indicated above.

The X-ray mineralogy study was supplemented by petrographic examination of the decalcified $>20 \mu$ and 2-20 $\mu$ residues. Microscopic examinations of the coarsergrained fractions helped to distinguish between authigenic and detrital components. In this paper the term "detrital" refers to grains derived from preexisting rocks. Individual detrital grains exhibit various degrees of angularity, and this morphology can be attributed to mechanical breakage or chemical solution. The term "authigenic" refers to discrete or aggregated grains which are interpreted to have formed in situ.

Drilling mud with montmorillonite and barite was utilized as follows on Leg 21: Hole 203-between Cores 4 and 5; Hole 205-between Cores 21 and 22; Hole 207A-between Cores 30 and 31, 39 and 40, 42 and 43, 44 and 45, 46 and 47, 49 and 50; Hole 207C-between Cores 20 and 21; Hole 209-between Cores 9 and 10, 18 and 19, 27 and 28, 31 and 32; Hole 210-between Cores 37 and 38. Of the cores sampled for X-ray diffraction analyses, only samples from Hole 207A, Core 47; Hole 209, Cores 27 and 32 may have been directly exposed to drilling mud, but none of these samples show traces of barite or inordinate concentrations of montmorillonite.

\section{RESULTS AND DISCUSSION}

\section{Site 203}

Site 203 is located in a sediment pond in the southern end of the Lau Basin. This basin is one of a series of ridges and basins that characterizes the eastern margin of the Tasman Plate; 20.3 meters of Pliocene and Pleistocene, nannofossil-rich, glass shard ash were recovered from 409 meters of sediment penetrated at this site.

Two samples from two cores were submitted for X-ray diffraction analyses. The age, lithology, stratigraphic 
position, and X-ray results of these samples are summarized in Tables 1 and 5.

The samples from Cores 3 and 4 consist of a calcareous plagioclase-montmorillonite suite containing volcanic glass and the mafic minerals augite and magnetite. Plagioclase and subordinate, clear quartz occur as angular to subangular, silt and fine sand-sized detrital grains. Samples from Cores 3 and 4 are mineralogically similar, with the exception that the sample from Core 3 has abundant, unaltered, colorless, volcanic glass and no mafic minerals, whereas the Core 4 sample has less volcanic glass and contains augite and magnetite. The fresh glass shards have refractive indexes between 1.512 and 1.522 , which suggests that they are approximately andesitic in composition. The presence of andesitic glass in Cores 3 and 4 contrasts with the presence of olivine, basaltic glass, and basalt cobbles reported by shipboard scientists in Core 5 (Site 203 Hole Summary).

Pliocene-Pleistocene samples from Site 203 are mineralogically similar to Middle Miocene through Pleistocene samples from Site 205 in the South Fiji Basin.

\section{Site 204}

Site 204 is located on the abyssal plain of the Pacific Plate approximately $120 \mathrm{~km}$ east of the Tonga-Kermadec Trench; 49.4 meters of ?Oligocene through Quaternary, volcanic shard-bearing, silty clays and Cretaceous volcanogenic sandstones and conglomerates were recovered from 147 meters of sediment penetrated at Site 204.

The sedimentary section was divided into three informal lithologic units, Units 1 to 3 (Site 204 Hole Summary). Eight samples from five cores in Unit 1 were submitted for $\mathrm{X}$-ray diffraction analyses. The age, lithology, stratigraphic position, and $\mathrm{X}$-ray mineralogy results of these samples are summarized in Tables 1 and 6.

Samples from Cores 1 to 3 are mineralogically variable. Plagioclase, montmorillonite, and phillipsite each dominate in one or more bulk and 2-20 $\mu$ samples. The percentages of subordinate minerals are also variable. Plagioclase and quartz occur as subangular, silt and fine sand-sized, detrital grains. Montmorillonite consistently predominates in $<2 \mu$ fractions. Unaltered, colorless volcanic glass occurs in all samples. The fresh glass shards have refractive indexes between 1.510 and 1.514, suggesting an andesitic to dacitic composition.

Samples from Cores 4 and 5 are mineralogically uniform. The bulk and $2-20 \mu$ samples are all dominated by $\mathrm{K}$-feldspar, and the $<2 \mu$ fractions are dominated by montmorillonite and K-feldspar. The abundant occurrence of K-feldspar in deep-sea sediments is unique. The feldspars occur as subangular to subrounded, silt-sized grains. Most grains are unaltered, but some grain surfaces appear to be etched. These grain textures suggest a detrital origin. However, the feldspars could be authigenic, with their original textures having been modified by subsequent abrasion and alteration. Future work on these samples using thin sections from impregnated sediments could help to resolve this question.

Cores 1 to 3 and 4 to 5 are easily distinguished from other on the basis of X-ray mineralogy. The variable plagioclase-montmorillonite-phillipsite-quartz suite of Cores 1 to 3 contrasts sharply with the K-feldsparmontmorillonite suite of Cores 4 and 5 .

Site 204 was drilled on the Pacific plate. The mineral assemblages recovered in Site 204 sediments have not been detected in sediments from other Leg 21 sites, which were all drilled on the Tasman plate.

\section{Site 205}

Site 205 is located on the east margin of the South Fiji Basin, adjacent to the western flank of the Lau Ridge. The Lau Ridge and South Fiji Basin are part of a series of ridges and depositional basins that characterize the eastern margin of the Tasman plate. 134.7 meters of Middle Oligocene through Pliocene, ash-bearing, calcareous and argillaceous oozes were recovered from 355 meters of sediment penetrated at this site.

The sedimentary section was divided into informal lithologic Units 1 to 4 (Site 205 Hole Summary). Twenty-six samples from 17 cores in Units 1 to 3 were submitted for X-ray diffraction analyses. The age, lithology, stratigraphic position, and X-ray mineralogy results of these samples are summarized in Table 1.

\section{Unit 1}

Late Miocene to Quaternary sediments of Unit 1 are characterized by a plagioclase-phillipsite-montmorillonite suite containing subordinate mafics (augite and magnetite) and unaltered, colorless, volcanic glass. Fresh glass shards have refractive indexes between 1.510 and 1.522 , suggesting an andesitic to dacitic composition. Plagioclase and quartz occur as subangular, silt-sized, detrital grains.

\section{Unit 2}

Middle and Late Miocene sediments of Unit 2 are characterized by a plagioclase-mafic-montmorillonite lithogenous suite in which biogenous calcite commonly dilutes the lithogenous components. Plagioclase and augite occur as angular to subangular, silt-sized grains, while magnetite grains are subrounded. Samples from Unit 2 contain variable amounts of unaltered, colorless, volcanic glass. Glass shards in Unit 2 are slightly more basic (refractive index, 1.514-1.528) than glass shards in Unit 1.

\section{Unit 3}

Late Oligocene sediments of Unit 3 are characterized by a calcite-montmorillonite-plagioclase suite with subordinate amounts of the zeolites phillipsite and clinoptilolite. Fresh glass shards are not common in samples from Unit 3. Here, montmorillonite aggregates may represent devitrified volcanic ash. Some bulk and $2-20 \mu$ samples contain amber to colorless, round to subround, silt-sized, microcrystalline aggregates. These grains have refractive indexes between 1.500 and 1.530 and exhibit a first-order gray interference color. Bulk and 2-20 $\mu$ samples containing these amber grains contain $70 \%$ to $80 \%$ montmorillonite. Irregular clots of colloidal clays were not observed in microscopic examination. The amber-colored grains of aggregated microcrystallites may represent partially devitrified glass 
shards in which authigenic montmorillonite displays the relict shard morphology.

Several observations can be made regarding the mineralogic sequence at Site 205:

1. There are several pronounced mineralogic trends in the Late Oligocene through Quaternary sediments which transgress lithologic unit boundaries. Quartz is generally in low concentration throughout the section. Plagioclase and magnetite diminish with depth. Montmorillonite increases with depth and constitutes nearly $100 \%$ of the $<2 \mu$ fraction at the base of the section.

2. Middle Miocene and younger Units 1 and 2 are separated from Late Oligocene Unit 3 by a major disconformity. Two mineralogic changes can be associated with this hiatus: (a) detrital augite was not detected in samples below the disconformity; and (b) detrital plagioclase is greatly reduced in samples below the disconformity, particularly in the $2-20 \mu$ and $<2 \mu$ fractions.

3 . No pronounced mineralogic change occurs at the boundary between Units 1 and 2, except for an apparent decrease in the abundance of phillipsite in Unit 2.

4. Fresh, andesitic glass is abundant in Units 1 and 2. Fresh glass is not common in Unit 3.

5. Biogenous calcite is variable in Units 1 and 2. Calcite is abundant in Unit 3.

6. Minor, detrital quartz in Units 1 and 2 contains very few inclusions.

Unit 3 is easily distinguished from Units 1 and 2 on the basis of X-ray mineralogy. Late Oligocene sediments below the disconformity are dominated by biogenous calcite, and contain no mafics and little fresh, volcanic glass. This mineral suite contrasts with the Middle Miocene-Quaternary Units 1 and 2 which contain abundant, unaltered, andesitic glass and mafics, and variable biogenous calcite.

Middle Miocene through Quaternary sediments at Site 205 are mineralogically similar to Pliocene and Pleistocene sediments at Site 203 in the Lau Basin.

\section{Site 206}

Site 206 is located near the southern end of the New Caledonia Basin east of the Lord Howe Rise, 243.6 meters of Middle Paleocene through Late Pleistocene calcareous oozes were recovered from 734 meters of sediment penetrated in Holes 206 and $206 \mathrm{C}$.

The sedimentary section at Site 206 was separated into seven informal lithologic units, Units 1A, 1B, 1C, 1D, 2, 3 and 4 (Site 206 Hole Summary). Samples were submitted from each lithologic unit. Twelve samples from Units 1A-1D and Unit 2 were submitted from Hole 206, and 23 samples from Units 2 to 4 were submitted from Hole 206C. The age, lithology, stratigraphic position, and X-ray mineralogy results of these samples are summarized in Tables 2, 8, and 9 .

Units 1 to 4 are difficult to distinguish from each other on the basis of the X-ray mineralogy of bulk samples. The entire stratigraphic section penetrated at Site 206 is highly calcareous, and the calcite dilutes the lithogenous components. Mineralogic distinctions between the lithogenous units can be made only in the decalcified $2-20 \mu$ and $<2 \mu$ fractions, although in Unit 1 there frequently was insufficient $2-20 \mu$ residue remaining for X-ray diffraction analyses following decalcification.

\section{Units 1 and 2}

Middle Oligocene-Late Pleistocene Units 1 and 2 are mineralogically similar. Both units exhibit a downhole increase in montmorillonite and a downhole decrease in mica, chlorite, detrital plagioclase, and $<20 \mu$ detrital, silt and fine sand-sized quartz. No significant changes in these trends occur at the boundary between Units 1 and 2. Both Units contain minor to abundant kaolinite $(2-20 \mu$ and $<2 \mu$ fractions), although kaolinite decreases in Unit 2 . Units 1 and 2 contain consistently large amounts of angular, $2-20 \mu$, detrital quartz.

\section{Unit 3}

Middle and Late Eocene sediments in Unit 3 are mineralogically similar to Units 1 and 2 . Biogenous calcite predominates in the bulk samples. The following differences occur in Unit 3: (1) the amount of angular, $2-20 \mu$ detrital quartz is much lower in Unit 3 than in Units 1 and 2 ; (2) abundant montmorillonite occurs in the $2-20 \mu$ fractions of Unit 3; and (3) barite occurs as minor components of Unit 3, 2-20 $\mu$ fractions.

\section{Unit 4}

Early to Middle Eocene Unit 4 is also dominated by biogenous calcite, but is distinguished from overlying lithologic units by the presence of authigenic cristobalite, tridymite, and abundant clinoptilolite.

Several observations can be made regarding the mineralogic sequence at this site:

1) There are two mineralogic trends in Early Eocene through Late Pleistocene sediments: (a) a downhole increase in montmorillonite; and (b) a downhole decrease in detrital lithogenous components.

2) Middle Oligocene through Late Pleistocene sediments of Units 1 and 2 are separated from Early through Late Eocene Units 3 and 4 by a major disconformity. Three mineralogic changes can be associated with this hiatus: (a) an abrupt decrease in the $2-20 \mu$ detrital quartz in samples below the disconformity; (b) an abrupt increase in montmorillonite in $2-20 \mu$ samples below the disconformity; and (c) the absence of barite in samples overlying the disconformity.

3) No pronounced mineralogic changes occur at the boundary between Units 1 and 2. Mineralogic trends established in Unit 1 are continued downhole in Unit 2. Kaolinite, however, is consistently abundant in Unit 1 but occurs rarely in Unit 2 and not at all in sediments below the disconformity. No pronounced mineralogic changes occur at the boundary between Units 3 and 4 .

4) Fresh, unaltered, volcanic glass is not common in sediments at Site 206. However, the morphology of some cristobalite-tridymite grains in Unit 4 suggests that some of the authigenic silica in the Early to Middle Eocene rocks may be the product of devitrified glass shards.

5) Biogenous calcite dominates all bulk samples at Site 206. 
6) Many detrital quartz grains in Middle Oligocene through Pleistocene Units 1 and 2 contain abundant inclusions.

Lithologic units at Site 206 can be distinguished from each other only on the basis of changes in mineralogy in the decalcified $2-20 \mu$ and $<2 \mu$ fractions.

The mineralogic sequence at Site 206 is generally similar to time-equivalent mineralogic sequences at Sites 207, 208, 209, and 210.

\section{Sites 207 and 208}

Sites 207 and 208 are located on the Lord Howe Rise. Site 207 was drilled on the southern end of the rise crest, near the Challenger Plateau; 38 meters of Plio-Pleistocene calcareous ooze were recovered from 48 meters of sediment penetrated in Hole 207, and 212.3 meters of Late Cretaceous (Maastrichtian) through Early Pleistocene sediment were recovered from 513 meters of section penetrated in Hole 207A. Site 208 was drilled on the north end of the Lord Howe Rise; 255.4 meters of Late Cretaceous (Maastrichtian) through Late Pleistocene sediment were recovered from 594 meters of section penetrated at Site 208.

The sedimentary section at Site 207 was divided into informal lithologic Units 1 to 5 (Site 207 Hole Summary). Five samples were submitted for X-ray diffraction analyses from Units 1, 3, and 5. The age, lithology, stratigraphic position, and X-ray mineralogy results of these samples are summarized in Tables 3,10 , and 11 . The sedimentary section at Site 208 was divided into lithologic Units 1 and 2 (Site 208 Hole Summary), but samples were submitted from Unit 2 only. The age, lithology, stratigraphic position, and X-ray mineralogy results of these three samples are summarized in Tables 2 and 12.

One sample of Pleistocene sediment was submitted from Hole 207. The lithogenous components consist mainly of detrital quartz and plagioclase, and mica; kaolinite, chlorite, and montmorillonite are minor. Abundant calcite dilutes the lithogenous components in the bulk sample.

Paleocene and Maastrichtian rocks at Site 207 consist of siliceous siltstones and claystones and vitrophyric rhyolite tuffs or flows (Site 207 Hole Summary). Siltstones and claystones in Unit 3 are dominated by cristobalite and tridymite with subordinate montmorillonite. The siliceous siltstones are underlain by rhyolitic rocks which are also dominated by cristobalite and montmorillonite. The presence of these minerals and their abundance suggest that the original volcanogenic rocks are highly altered. Minor quartz grains in both the rhyolitic rocks and the overlying siltstones are usually devoid of inclusions.

At Site 208, Maastrichtian to Middle Eocene sediments of Unit 2 are dominated by biogenous calcite which dilutes the lithogenous components. The lithogenous constituents include cristobalite and clinoptilolite, montmorillonite, and subangular to subrounded, silt-sized, clear, detrital quartz. Fresh volcanic glass is not common. Some angular and subrounded cristobalite grains may represent partially devitrified glass shards.

The Maastrichtian and Early Paleocene vitrophyric rhyolites and siliceous siltstones at Site 208 have not been recognized at other sites on Leg 21. However, their cristobalite-tridymite-montmorillonite lithogenous suite occurs in Maastrichtian, Paleocene, and Eocene calcitemontmorillonite oozes at Sites 206 and 208. The Pleistocene sample at Site 207 is mineralogically similar to the Pleistocene sediments at Sites 206, 208, 209, and 210.

\section{Site 209}

Site 209 is located on the northeast margin of the Queensland Plateau in the Coral Sea; 77 meters of Middle Eocene through Pleistocene calcareous oozes and calcareous silty sands and cherts were recovered from 344 meters of sediment penetrated at this site.

The sedimentary section was divided into informal lithologic Units 1 to 3 (Site 209 Hole Summary). Sixteen samples from 16 cores in Units 1 to 3 were submitted for $\mathrm{X}$-ray diffraction analyses. The age, lithology, stratigraphic position, and $\mathrm{X}$-ray mineralogy résults of these samples are summarized in Tables 3 and 13.

Units 1 to 3 are difficult to distinguish from each other on the basis of the X-ray mineralogy of bulk samples. The entire stratigraphic section penetrated at Site 209 is highly calcareous, and the calcite dilutes the lithogenous components. Mineralogical distinctions between the lithologic units can be made only in the decalcified $2-20 \mu$ and $<2 \mu$ fractions. However, in Unit 1 there was frequently insufficient residue remaining for X-ray diffraction analysis following decalcification.

\section{Unit 1}

Late Oligocene through Pleistocene sediments of Unit 1 are dominated by biogenous calcite, and contain so few silt-sized, acid-insoluble components that insufficient $2-20 \mu$ residue remained for X-ray diffraction analysis following decalcification. Residues $<20 \mu$ contain (a) angular to subangular, silt to fine sand-sized, detrital quartz; (b) minor, fresh, colorless, volcanic glass (refractive index $<1.550$ ); and (c) rounded, devitrified glass shards. The $<2 \mu$ fractions are dominated by montmorillonite, but kaolinite, mica, plagioclase, and quartz are well represented. Underlying Units 2 and 3 do not contain kaolinite.

\section{Units 2 and 3}

Middle and Late Eocene Units 2 and 3 are easily distinguished from Unit 1 on the basis of X-ray mineralogy, but they are not easily distinguished from each other. Calcite content is variable, but calcite dominates all bulk samples. The silica phases quartz, cristobalite, and tridymite dominate the decalcified $2-20 \mu$ and $<2 \mu$ fractions, along with subordinate plagioclase and clinoptilolite. Plagioclase and quartz occur as subangular to subrounded, silt and fine sand-sized, detrital grains. The quartz grains contain a few to numerous inclusions. Cristobalite (refractive index 1.484) occurs as subangular to subrounded, microcrystalline aggregates.

Several observations can be made regarding the mineralogic sequence at Site 209:

1) The major mineralogic trend at Site 209 is a downhole increase in detrital lithogenous components.

2) Late Oligocene through Pleistocene sediments of Unit 1 are separated from Middle and Late Eocene Units 2 
and 3 by a major disconformity. One mineralogic change can be associated with this hiatus: authigenic cristobalite, tridymite, and clinoptilolite occur in samples below the disconformity, but do not occur above it.

3) No mineralogic changes occur at the boundary between Units 2 and 3. Mineralogic changes occur across the boundary between Units 1 and 2, but these are associated with the disconformity and are described above.

4) Unaltered volcanic glass is not common in sediments at Site 209. Fresh glass, andesitic to basaltic in composition (refractive index 1.550), was observed in Unit 1, accompanied by altered glass shards. 209.

5) Biogenous calcite dominates all bulk samples at Site

6) Detrital quartz grains at Site 209 contain few to numerous inclusions.

7) Kaolinite is a consistently minor to abundant component of Miocene and younger sediments of Unit 1 .

Lithologic units at Site 209 can be distinguished from each other only on the basis of changes in mineralogy in the decalcified $2-20 \mu$ and $<2 \mu$ fractions.

The mineralogic sequence at Site 209 is generally similar to time-equivalent sequences at Sites 206, 207, 208, and 210 .

\section{Site 210}

Site 210 is located in the center of the Coral Sea abyssal plain; 262.3 meters of Middle Eocene through Late Pleistocene chalks, oozes, and turbidites were recovered from 711 meters of sediment penetrated at this site.

The sedimentary sequence at Site 210 was divided into informal lithologic Units 1 to 5 (Site 210 Hole Summary). Fifty-seven samples from 38 cores in Units 1 to 5 were submitted for X-ray diffraction analyses. The age, lithology, stratigraphic position, and X-ray mineralogy results of these samples are summarized in Tables 4 and 14.

Lithologic Units 1 and 2 are easily distinguished from Units 3,4 , and 5 on the basis of X-ray mineralogy.

\section{Units 1 and 2}

Late Miocene through Late Pleistocene Unit 1 consists of turbidite beds alternating with interbeds of calcareous ooze. Some calcareous samples contain abundant aragonite as well as calcite. We cannot determine whether the aragonite represents in situ authigenic or skeletal aragonite, or whether the aragonite represents allochthonous skeletal aragonite brought into Site 210 by turbidity currents.

The lithogenous content of Unit 1 is best observed in the decalcified $2-20 \mu$ and $<2 \mu$ fractions, where the diluting effect of the carbonates is eliminated. As seen in the 2-20 $\mu$ fractions, the mineralogic composition of the detrital lithogenous components remains uniform despite their mechanism of deposition (suspension deposition versus turbidity current deposition). Quartz, plagioclase, and mica are the major components of both turbidites and calcareous interbeds. Plagioclase and quartz occur as subangular to subrounded, silt and sand-sized, detrital grains. The quartz contains abundant inclusions. K-feldspar and amphibole persist in minor amounts. The $<2 \mu$ fractions are dominated by montmorillonite, but mica, quartz, and minor to abundant kaolinite and chlorite persist throughout Unit 1 . Kaolinite and chlorite do not occur in underlying units.

Two samples submitted from Unit 2 (a nonturbidite unit) are mineralogically similar to Unit 1.

\section{Units 3,4 , and 5}

One sample from Unit 3 is mineralogically similar to Unit 4, even though Unit 3 is separated from Unit 4 by an Early Oligocene disconformity.

Two samples were submitted from Unit 4. The samples are dominated by biogenous calcite and are mineralogically similar to youngest sediments in Unit 5 .

Early and Middle Eocene Unit 5 has abundant biogenous calcite. The lithogenous content of Unit 5 is best observed in the decalcified $2-20 \mu$ and $<2 \mu$ fractions, where the diluting of the carbonates is eliminated. The general mineralogic trend in lithogenous components in Unit 5 is a downhole increase in authigenic silicates (clinoptilolite, cristobalite and tridymite, and quartz). Montmorillonite decreases downhole in Unit 5. Kaolinite does not occur in Unit 5. The quartz content increases abruptly in the $>20 \mu$, $2-20 \mu$, and $<2 \mu$ fractions in Core 43. Microscopic examination indicates that the abundance of angular to subangular, silt to fine sand-sized, detrital quartz is nearly the same in samples throughout Unit 5. The increase in quartz below Core 42 is attributed to the occurrences of silt and sand-sized, subangular to subrounded, grains of microcrystalline aggregates. These grains have refractive index between 1.544 and 1.550 and probably represent authigenic quartz. Authigenic development thus probably accounts for the increase in quartz below Core 42 .

Unit 5 can be separated into three distinct zones on the basis of diagenetic components. These zones overlap only slightly. Samples of $2-20 \mu$ and $<2 \mu$ from Cores 36 through 38 primarily contain clinoptilolite. Cores 39 through 42 mainly contain cristobalite and tridymite, with clinoptilolite absent. Cores 43 through 50 contain authigenic quartz, with clinoptilolite and, cristobalite and tridymite absent.

Several observations can be made regarding the mineralogic sequence at Site 210:

1) Early Oligocene through Pleistocene Units 1 and 2 disconformably overlie Early to Late Eocene sediments of Units 3, 4, and 5. Two mineralogical changes can be associated with this hiatus: (a) a general decrease in detrital lithogenous components in sediments below the disconformity; and (b) the presence of authigenic silicates and barite in sediments below the disconformity.

2) No pronounced mineralogic changes occur at the boundaries between Units 1 and 2 and between Units 3, 4, and 5. Mineralogic changes occur across the boundary between Units 2 and 3, but these are associated with the disconformity and are described above.

3) Unaltered volcanic glass is not common in Site 210 sediments as in Site 209.

4) Biogenous calcite dominates all bulk samples at Site 210.

5) Many detrital quartz grains in Early Eocene through Pleistocene Units 1 to 5 contain abundant inclusions.

6) Kaolinite and chlorite are consistently minor to abundant in Early Miocene and younger sediments of Unit 1. 
Lithologic Units at Site 210 are most easily distinguished from each other on the basis of changes in the mineralogy of the decalcified $2-20 \mu$ and $<2 \mu$ fractions.

The mineralogic sequence at Site 210 is generally similar to time-equivalent mineralogic sequences at Sites 206, 207, 208 , and 209.

\section{SUMMARY}

Five generalized sedimentary sequences can be recognized at the sites on Leg 21. Grouping data in such a manner represents a first-order step toward recognizing different sedimentologic provinces on the basis of their mineralogy. Stratigraphic intervals represented by the same sedimentary sequence have similar mineralogic successions (as determined by X-ray mineralogy data and by microscope studies described in this report) and similar lithologic descriptions (as presented by Leg 21 Hole Summaries). The following generalized sedimentary sequences are recognized:

2) Site 204-an ?Oligocene through Quaternary pelagic sequence.

2) Sites 203 and 205-a Middle Miocene through ?Recent volcaniclastic sequence.

3) Sites 206, 207, and 208-a Maastrichtian through Eocene siliceous-calcareous sequence overlain disconformably by

4) a Late Eocene (or younger) through Pleistocene calcareous-terrigenous-volcanic sequence.

5) Sites 209 and 210-an Early Eocene through Pleistocene calcareous-terrigenous sequence. Terrigenous input was primarily by aeolian and suspension transport (Site 209), or by aeolian and suspension transport augmented by turbidity current deposition (Site 210).

\section{Site 204}

Site 204 is located on the western margin of the Pacific plate adjacent to the Tonga-Kermadec Trench. ?Oligocene to Quaternary sediments consist of montmorillonite clays containing detrital lithogenous components. The detrital suite is compositionally variable and consists of subangular to subrounded, silt and sand-sized plagioclase, K-feldspar, mica, augite, and clear quartz. Andesitic to acidic glass shards occur in Pliocene to Quaternary samples.

?Oligocene through Quaternary sediments at Site 204 can be characterized as a pelagic suite: sediments deposited in the open ocean where sedimentation is not dominated by local, continental, terrigenous input.

Tertiary pelagic sediments at this site disconformably overlie Cretaceous volcanogenic sandstone and conglomerate for which we have no X-ray mineralogy samples.

\section{Sites 203 and 205}

Sites 203 and 205 are located in the Lau and South Fiji basins, respectively. Middle Miocene through Recent sediments consist of montmorillonite clays containing abundant, detrital, lithogenous components. Unaltered, andesitic to dacitic, volcanic glass is abundant and is volumetrically one of the most important constituents. Biogenous calcite content is variable. The detrital suite is compositionally uniform and consists of subangular to subrounded, silt and sand-sized plagioclase, augite, magnetite, and minor amounts of clear quartz. Only traces of mica, kaolinite, and chlorite are present. Authigenic phases include phillipsite, clinoptilolite, and rare analcite. Authigenic cristobalite, tridymite, and quartz are not present.

Middle Miocene and younger sediments at Site 205 are underlain disconformably by Middle and Late Oligocene nanno oozes. These older sediments are dominated by biogenous calcite and montmorillonite and contain no augite and only traces of magnetite. Detrital plagioclase decreases downhole; fresh, volcanic glass is not abundant in the samples submitted for X-ray diffraction analysis.

We suggest that Middle Miocene and younger sediments at Sites 203 and 205 represent volcaniclastic sequences containing primarily (1) pyroclastics, (2) detrital, volcanogenic material, (3) variable amounts of biogenous calcite, and (4) minor amounts of quartz and mica. The sequences possibly accumulated in depositional basins adjacent to andesitic volcanic islands. Rates of volcanicalstic sedimentation were significantly greater than pelagic sedimentation rates at Site 204.

We cannot characterize the depositional conditions of Late Oligocene and older sediments at Site 205.

\section{Sites 206, 207, and 208}

Site 206 is located in the New Caledonia Basin, while Sites 207 and 208 are located on the south and north ends of the Lord Howe Rise, respectively. Late Cretaceous (Maastrichtian) and Early Paleocene through Middle and Late Eocene sediments at Sites 206, 207, and 208 constitute siliceous-calcareous sequences. These sediments are overlain disconformably by Middle Oligocene (or younger) through Pleistocene calcareous-terrigenousvolcanic sequences.

The succession of sedimentary sequences is best observed at Site 206, where sampling for X-ray mineralogy analysis by shipboard scientists was most extensive.

1) The siliceous-calcareous sequence: At Site 206 the siliceous-calcareous sequence is Middle Paleocene through Late Eocene in age and consists of siliceous, calcitemontmorillonite oozes. The sequence is dominated by biogenous calcite. The lithogenous components are dominated by montmorillonite and cristobalite and include minor amounts of clear, detrital quartz. Authigenic silica (cristobalite and tridymite) and authigenic clinoptilolite in the Paleocene and Eocene sediments can be interpreted to be products of either devitrified biogenous silica or devitrified volcanic glass. Since devitrified glass shards are present and because we observed no relict skeletal textures in the cristobalite grains, we favor a volcanic source for much of the authigenic silica.

2) The calcareous-terrigenous-volcanic sequence: This sequence disconformably overlies the siliceous-calcareous sequence at Site 206. At Site 206 the sequence began in the Middle Oligocene and continued through the Pleistocene. However, the base of the sequence ranges from Late Eocene (Site 207) to Late Oligocene (Site 208).

The calcareous-terrigenous-volcanic sequence is dominated biogenous calcite. Detrital components $>20 \mu$ generally consist of silt and fine sand-sized quartz grains 
containing few to abundant inclusions. Fine silt-sized, 2-20 $\mu$ detrital components are more diverse: detrital quartz, containing few to abundant inclusions, dominates the fine silt components, followed by plagioclase, mica, and chlorite (as at Sites 209 and 210) and minor volcanic glass. The $<2 \mu$ fractions are dominated by montmorillonite, mica, kaolinite, chlorite, and detrital quartz and plagioclase are all well represented in the $<2 \mu$ fractions of Miocene and younger sediments (as at Sites 209 and 210).

The generalized sedimentary sequences recognized at Site 206 can be extended to Sites 207 and 208, even through the sediments at these sites were not extensively sampled for X-ray diffraction analyses. The sedimentary sequences can be correlated between Sites 206, 207, and 208 because (1) a small number of X-ray samples from Sites 207 and 208 are mineralogically similar to samples from Site 206; (2) lithologic descriptions of lithologic units at Sites 207 and 208 are very similar to lithologic descriptions at Site 206; and (3) the lithologic units occupy the same relative stratigraphic positions at Sites 206, 207, and 208.

At Site 207, Maastrichtian rocks include cristobalitebearing siltstones underlain by volcanogenic rocks. The volcanogenic rocks consist of rhyolitic tuffs or flows (Site 207 Hole Summary) which have been highly altered to montmorillonite and cristobalite. These volcanic rocks are overlain by siltstones dominated by cristobalite and containing tridymite, montmorillonite, and detrital plagioclase and clear quartz. Cristobalite and tridymite may represent authigenic products of devitrified volcanic glass, or they may represent detrital grains derived from underlying, altered, volcanic rocks.

It is tempting to suggest that the rhyolitic rocks and siliceous siltstones at Site 207 are part of a regional lithologic sequence at Sites 206, 207, and 208 which grades upward from Maastrichtian volcanogenic rocks and volcaniclastic siltstones (Site 207) into Maastrichtian and younger calcareous sediments of the siliceous-calcareous sequence (Sites 206, 207, and 208), with a reduction in volcaniclastic materials with decreasing age. Thus, the siliceous-calcareous may represent the end of a waning volcaniclastic episode in the Lord Howe Rise-New Caledonia Basin region. In this way, the siliceous-calcareous sequence and underlying volcanics may represent a devitrified, volcaniclastic sequence which may be similar in origin (but not in mineralogy) to the Miocene and younger volcaniclastic sequences at Sites 203 and 205. This tentative interpretation is supported by the abundance of montmorillonite and authigenic silica in the siliceous-calcareous sequence and in the underlying siltstones and volcanics. However, we cannot confidently establish a stratigraphic or genetic relationship between the Maastrichtian through Eocene siliceouscalcareous sequence and the underlying volcanics and siltstones because (1) these latter rocks were not penetrated at Sites 206 and 208, and (2) because we do not have X-ray samples from the siliceous-calcareous sequence at Site 207. Hence, we cannot conclusively determine the depositional conditions of the siliceous-calcareous sequence.
Middle Oligocene (or younger) through Pleistocene calcareous-terrigenous-volcanic sequences at Sites 206, 207, and 208 can be characterized as sediments deposited in the open ocean, with detrital sediments dominated by local, continental, terrigenous input (contrast with Site 204). The terrigenous input at these sites is represented by kaolinite and chlorite and by abundant detrital quartz, plagioclase, and mica. The quartz grains contain few to abundant inclusions (compare with Sites 209 and 210). Volcanic input is represented by the sporadic presence of glass shards (contrast with Sites 209 and 210, in which glass shards are not common in the calcareous-terrigenous sequence).

\section{Sites 209 and 210}

Sites 209 and 210 are located in the Coral Sea, on the margin of the Queensland Plateau and on the abyssal plain, respectively. Sediments at Sites 209 and 210 constitute calcareous-terrigenous sequences. Terrigenous components were transported and deposited primarily by aeolian and suspension processes at Site 209, while turbidity currents augmented aeolian and suspension transport of terrigenous components in Miocene and younger sediments at Site 210.

The Eocene through Pleistocene sediments at Sites 209 and 210 are highly calcareous. The detrital lithogenous suite in the calcareous-terrigenous sequence at Sites 209 and 210 remains essentially unchanged during Eocene through Pleistocene time, with a few important exceptions. The $>20 \mu$ and $2-20 \mu$ detrital fractions are dominated by subangular to subrounded, fine silt to sand-sized quartz, plagioclase, and mica. The quartz contains few to abundant inclusions. Montmorillonite, detrital quartz, plagioclase, and mica are all well represented in the $<2 \mu$ fractions in the Eocene to Pleistocene sediments (as at Sites 206, 207, and 208). However, persistent, minor to abundant kaolinite and chlorite are restricted to Miocene and younger sediments at Sites 209 and 210 (as at Sites 206, 207, and 208).

The lithogenous components of Eocene sediments at both Sites 209 and 210 are dominated by authigenic silicates: quartz, cristobalite + tridymite, and clinoptilolite. These authigenic phases occur below an Oligocene disconformity.

Early and Middle Eocene through Pleistocene calcareousterrigenous sequences at Sites 209 and 210 represent sediments deposited in an open ocean, with detrital sediments dominated by local, continental, terrigenous input (contrast with Site 204). Turbidites in Late Miocene and younger sediments at Site 210 indicate relative proximity of the Coral Sea abyssal plain to a slope and continental source terrain.

\section{ACKNOWLEDGMENT}

The writers wish to acknowledge the excellent work of Nicki D. Coursey in the analysis and the quantification of X-ray diffraction data, of Paul D. Johnson in X-ray data acquisition and data processing, and of Tom W. Halverson, Jr. in sample preparation. 
TABLE 1

Summary of X-ray Mineralogy Samples, Sample Depths, Lithology, Age, and X-ray Diffraction Results,

Sites 203, 204, 205

\begin{tabular}{|c|c|c|c|c|c|c|c|c|c|c|c|c|c|c|}
\hline Sample & $\begin{array}{c}\text { Sample } \\
\text { Depth in } \\
\text { Section }(\mathrm{cm})\end{array}$ & $\begin{array}{c}\text { Sample } \\
\text { Depth Below } \\
\text { Sea Floor (m) }\end{array}$ & Lithology & Age & $\begin{array}{l}\text { Bulk } \\
\text { Major } \\
\quad 1\end{array}$ & \multicolumn{2}{|c|}{$\begin{array}{c}\text { Sample } \\
\text { Constituent }\end{array}$} & $\begin{array}{l}2-20 \mu \\
\text { Major } \\
1\end{array}$ & \multicolumn{2}{|c|}{$\begin{array}{c}\text { Fraction } \\
\text { Constituent }\end{array}$} & $\begin{array}{l}<2 \mu \\
\text { Major } \\
1\end{array}$ & \multicolumn{2}{|c|}{$\begin{array}{l}\text { Fraction } \\
\text { Constituent }\end{array}$} & Comments \\
\hline $\begin{array}{l}203-3-4 \\
203-4-1\end{array}$ & $\begin{array}{l}120-122 \\
111-113\end{array}$ & $\begin{array}{l}113.7 \\
230.1\end{array}$ & $\begin{array}{l}\text { Nanno ooze and } \\
\text { volcanic ash }\end{array}$ & $\begin{array}{l}\text { Plio.- } \\
\text { Pleist. }\end{array}$ & $\begin{array}{l}\text { Calc. } \\
\text { Plag. }\end{array}$ & $\begin{array}{l}\text { Plag. } \\
\text { Calc. }\end{array}$ & $\begin{array}{l}\text { Mont. } \\
\text { Mafi.a }\end{array}$ & $\begin{array}{l}\text { Plag. } \\
\text { Plag. }\end{array}$ & $\begin{array}{l}\text { Quar. } \\
\text { Mafi. }\end{array}$ & Quar. & $\begin{array}{l}\text { Mont. } \\
\text { Mont. }\end{array}$ & $\begin{array}{l}\text { Plag. } \\
\text { Plag. }\end{array}$ & $\begin{array}{l}\text { Chlor. } \\
\text { Mafi. }\end{array}$ & $\mathrm{c}$ \\
\hline $\begin{array}{l}204-1-3 \\
204-2-3 \\
204-3 \\
204-3-1 \\
204-4-1 \\
204-4 \\
204-4 \\
204-5-6\end{array}$ & $\begin{array}{c}89-91 \\
73-75 \\
84-86 \\
140-142 \\
110-112 \\
86-88 \\
146-148 \\
130-132\end{array}$ & $\begin{array}{r}3.9 \\
11.2 \\
11.3 \\
49.4 \\
86.1 \\
90.4 \\
91.0 \\
102.8\end{array}$ & $\begin{array}{l}\quad \text { Unit } 1 \\
\text { Glass-bearing, silty, } \\
\text { pelagic clays }\end{array}$ & $\begin{array}{l}\text { ?Oligocene } \\
\text { through } \\
\text { Quaternary }\end{array}$ & $\begin{array}{l}\text { Plag. } \\
\text { Phil. } \\
\text { Mont. } \\
\text { Mont. } \\
\text { K-Fe. } \\
\text { K-Fe. } \\
\text { K-Fe. } \\
\text { K-Fe. }\end{array}$ & $\begin{array}{l}\text { Mica. } \\
\text { Plag. } \\
\text { Plag. } \\
\text { Plag. } \\
\text { Quar. } \\
\text { Calc. } \\
\text { Quar. } \\
\text { Quar. }\end{array}$ & $\begin{array}{l}\text { Quar. } \\
\text { Mica } \\
\text { Augi. } \\
\text { Mont. } \\
\text { Mont. } \\
\text { Mont. }\end{array}$ & $\begin{array}{l}\text { Plag. } \\
\text { Phil. } \\
\text { Insuffic } \\
\text { Mont. } \\
\text { K-Fe. } \\
\text { K-Fe. } \\
\text { K-Fe. } \\
\text { K-Fe. }\end{array}$ & $\begin{array}{l}\text { Quar. } \\
\text { Mica } \\
\text { cient resi } \\
\text { Plag. } \\
\text { Quar. } \\
\text { Quar. } \\
\text { Quar. } \\
\text { Quar. }\end{array}$ & $\begin{array}{l}\text { Mica } \\
\text { Quar. } \\
\text { idue } \\
\text { Mafi. } \\
\text { Mafi. } \\
\text { Mafi. } \\
\text { Mafi. }\end{array}$ & $\begin{array}{l}\text { Mont. } \\
\text { Mont. } \\
\text { Mont. } \\
\text { Mont. } \\
\text { K-Fe. } \\
\text { Mont. } \\
\text { Mont. } \\
\text { Mont. }\end{array}$ & $\begin{array}{l}\text { Plag. } \\
\text { Plag. } \\
\text { Anal. } \\
\text { Quar. } \\
\text { K-Fe. } \\
\text { K-Fe. } \\
\text { K-Fe. }\end{array}$ & & d \\
\hline $\begin{array}{l}205-2-1 \\
205-4 \\
205-3 C C \\
\end{array}$ & $\begin{array}{c}110-112 \\
80-82\end{array}$ & $\begin{array}{l}10.1 \\
14.3 \\
27.0 \\
\end{array}$ & $\begin{array}{l}\text { Unit } 1 \\
\text { Glass-bearing silty } \\
\text { clay and nanno ooze }\end{array}$ & $\begin{array}{l}\text { L. Mio. } \\
\text { to } \\
\text { ?Recent }\end{array}$ & $\begin{array}{l}\text { Plag. } \\
\text { Plag. } \\
\text { Phil. }\end{array}$ & $\begin{array}{l}\text { Mont. } \\
\text { Phil. } \\
\text { Plag. }\end{array}$ & $\begin{array}{l}\text { Mafi. } \\
\text { Quar. }\end{array}$ & $\begin{array}{l}\text { Plag. } \\
\text { Plag. } \\
\text { Phil. }\end{array}$ & $\begin{array}{l}\text { Mafi. }^{\text {a }} \\
\text { Phil. } \\
\text { Plag. }\end{array}$ & $\begin{array}{l}\text { Mont. } \\
\text { Mont. }\end{array}$ & $\begin{array}{l}\text { Mont. } \\
\text { Mont. } \\
\text { Mont. }\end{array}$ & $\begin{array}{l}\text { Plag. } \\
\text { Phil. } \\
\text { Phil. } \\
\end{array}$ & $\begin{array}{l}\text { Augi. } \\
\text { Plag. } \\
\text { Plag. }\end{array}$ & e \\
\hline $\begin{array}{l}205-5-3 \\
205-7-1 \\
205-8-2 \\
205-3 \\
205 C C \\
205-11-2 \\
205-12 C C \\
205-15-2 \\
205-3 \\
205 C C \\
205-16-3 \\
205-17-3 \\
205-23 C C \\
\end{array}$ & $\begin{array}{c}110-112 \\
52-54 \\
90-92 \\
104-106 \\
\\
105-107 \\
\\
110-112 \\
80-82 \\
\\
75-77 \\
100-102\end{array}$ & $\begin{array}{r}42.1 \\
56.5 \\
67.4 \\
69.0 \\
74.0 \\
97.6 \\
113.0 \\
133.6 \\
134.8 \\
140.0 \\
145.8 \\
155.0 \\
255.0 \\
\end{array}$ & $\begin{array}{l}\text { Unit } 2 \\
\text { Glass shard ash and } \\
\text { interbedded nanno } \\
\text { ooze }\end{array}$ & $\begin{array}{l}\text { M. and L. } \\
\text { Miocene }\end{array}$ & $\begin{array}{l}\text { Calc. } \\
\text { Calc. } \\
\text { Plag. } \\
\text { Plag. } \\
\text { Plag. } \\
\text { Plag. } \\
\text { Plag. } \\
\text { Plag. } \\
\text { Mont. } \\
\text { Mafi. } \\
\text { Plag. } \\
\text { Plag. } \\
\text { Plag. }\end{array}$ & $\begin{array}{l}\text { Plag. } \\
\text { Plag. } \\
\text { Calc. } \\
\text { Mafi. } \\
\text { Mafi. } \\
\text { Mafi. } \\
\text { Mafi. } \\
\text { Mont. } \\
\text { Plag. } \\
\text { Mont. } \\
\text { Calc. } \\
\text { Mont. } \\
\text { Calc. }\end{array}$ & $\begin{array}{l}\text { Mafi. } \\
\text { Mafi. } \\
\text { Calc. } \\
\text { Calc. } \\
\text { Calc. } \\
\text { Mont. } \\
\text { Mafi. } \\
\text { Mafi. } \\
\text { Mafi. } \\
\text { Mafi. } \\
\text { Mont. }\end{array}$ & $\begin{array}{l}\text { Plag. } \\
\text { Plag. } \\
\text { Plag. } \\
\text { Mont. } \\
\text { Plag. } \\
\text { Plag. } \\
\text { Plag. } \\
\text { Plag. } \\
\text { Mafi. } \\
\text { Plag. } \\
\text { Plag. } \\
\text { Plag. } \\
\text { Plag. }\end{array}$ & $\begin{array}{l}\text { K-Fe. } \\
\text { Mafi. } \\
\text { Mafi. } \\
\text { Plag. } \\
\text { Mafi. } \\
\text { Mafi. } \\
\text { Mafi. } \\
\text { Mafi. } \\
\text { Plag. } \\
\text { Mafi. } \\
\text { Mafi. } \\
\text { Mafi. } \\
\text { Mont. }\end{array}$ & $\begin{array}{l}\text { Mont. } \\
\text { Mont. } \\
\text { Mont. } \\
\text { Quar. } \\
\text { Mont. } \\
\text { Mont. }\end{array}$ & $\begin{array}{l}\text { Mont. } \\
\text { Mont. } \\
\text { Mont. } \\
\text { Mafi. } \\
\text { Mont. } \\
\text { Mont. } \\
\text { Mont. } \\
\text { Mont. } \\
\text { Mont. } \\
\text { Mont. } \\
\text { Mont. } \\
\text { Mont. } \\
\text { Mont. }\end{array}$ & $\begin{array}{l}\text { Plag. } \\
\text { Plag. } \\
\text { Plag. } \\
\text { Mont. } \\
\text { Plag. } \\
\text { Plag. } \\
\text { Plag. } \\
\text { Plag. } \\
\text { Plag } \\
\text { Plag. } \\
\text { Plag. } \\
\text { Plag. }\end{array}$ & $\begin{array}{l}\text { K-Fe. } \\
\text { Mafi. } \\
\text { Plag. }\end{array}$ & $\begin{array}{l}\text { Detrital quartz minor; } \\
\text { mica absent } \mathrm{f}^{\mathrm{f}}\end{array}$ \\
\hline $\begin{array}{l}205-24-3 \\
205 C C \\
205-25 C C \\
205-26-3 \\
205 C C \\
205-27 C C \\
205-28-1 \\
205-1 \\
205 C C \\
205-29-2 \\
205-3\end{array}$ & $\begin{array}{c}105-107 \\
139-141 \\
\\
70-72 \\
104-106\end{array}$ & $\begin{array}{l}277.5 \\
283.0 \\
292-0 \\
295.6 \\
301.0 \\
310.0 \\
311.0 \\
311.4 \\
319.0 \\
321.2 \\
323.0\end{array}$ & $\begin{array}{l}\text { Unit } 3 \\
\text { Nanno ooze with } \\
\text { subordinate litho- } \\
\text { genous constituents }\end{array}$ & $\begin{array}{l}\text { M. and L. } \\
\text { Oligocene }\end{array}$ & $\begin{array}{l}\text { Calc. } \\
\text { Calc. } \\
\text { Calc. } \\
\text { Calc. } \\
\text { Mont. } \\
\text { Calc. } \\
\text { Mont. } \\
\text { Calc. } \\
\text { Calc. } \\
\text { Calc. }\end{array}$ & $\begin{array}{l}\text { Mont. } \\
\text { Plag. } \\
\text { Mont. } \\
\text { Calc. } \\
\text { Mont. } \\
\text { Plag. } \\
\text { Mont. } \\
\text { Zeol.b }\end{array}$ & $\begin{array}{l}\text { Plag. } \\
\text { Mont. } \\
\text { Plag. } \\
\text { Mica. }\end{array}$ & $\begin{array}{l}\text { Insuffic } \\
\text { Mont. } \\
\text { Plag. } \\
\text { Mont. } \\
\text { Mont. } \\
\text { Mont. } \\
\text { Mont. } \\
\text { Mont. } \\
\text { Phil. } \\
\text { Phil. } \\
\text { Mont. }\end{array}$ & $\begin{array}{l}\text { cient res } \\
\text { Plag. } \\
\text { Mont. } \\
\text { Plag. } \\
\text { Plag. } \\
\text { Plag. } \\
\text { Plag. } \\
\text { Plag. } \\
\text { Clin. } \\
\text { Clin. } \\
\text { Plag. }\end{array}$ & $\begin{array}{l}\text { idue } \\
\text { Magn. } \\
\text { Quar. } \\
\text { Quar. } \\
\text { Mica. } \\
\text { Plag. } \\
\text { Plag. }\end{array}$ & $\begin{array}{l}\text { Mont. } \\
\text { Mont. } \\
\text { Mont. } \\
\text { Mont. } \\
\text { Mont. } \\
\text { Mont. } \\
\text { Mont. } \\
\text { Mont. } \\
\text { Mont. } \\
\text { Mont. } \\
\text { Mont. }\end{array}$ & $\begin{array}{l}\text { Quar. } \\
\text { Plag. } \\
\text { Mica } \\
\text { Quar. } \\
\text { Quar. } \\
\text { Plag. }\end{array}$ & Plag. & $\begin{array}{l}\text { Augite absent, traces of } \\
\text { devitrified glass }\end{array}$ \\
\hline
\end{tabular}

a Mafi. = Mafic minerals, augite and magnetite.

bZeol. = Zeolite minerals, clinoptilolite, phillipsite and analcite.

cAbundant andesitic glass, refractive index, 1.512-1.522

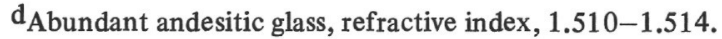

eAbundant andesitic glass, refractive index, 1.510-1.512.

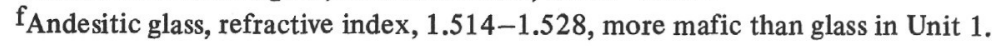


TABLE 2

Summary of X-ray Mineralogy Samples, Sample Depths, Lithology, Age, and X-ray Diffraction Results

Sites 206, 207, 208

\begin{tabular}{|c|c|c|c|c|c|c|c|c|c|c|c|c|c|c|}
\hline \multirow[b]{2}{*}{ Sample } & \multirow{2}{*}{$\begin{array}{c}\text { Sample } \\
\text { Depth in } \\
\text { Section }(\mathrm{cm})\end{array}$} & \multirow{2}{*}{$\begin{array}{c}\text { Sample } \\
\text { Depth Below } \\
\text { Sea Floor }(\mathrm{m})\end{array}$} & \multirow[b]{2}{*}{ Lithology } & \multirow[b]{2}{*}{ Age } & \multirow{2}{*}{$\begin{array}{c}\text { Bulk } \\
\text { Major } \\
1\end{array}$} & \multicolumn{2}{|c|}{$\begin{array}{l}\text { Sample } \\
\text { Constituent }\end{array}$} & \multirow{2}{*}{$\begin{array}{l}2-20 \mu \\
\text { Major } \\
1\end{array}$} & \multicolumn{2}{|c|}{$\begin{array}{c}\text { Fraction } \\
\text { Constituent }\end{array}$} & \multirow{2}{*}{$\begin{array}{l}<2 \mu \\
\text { Major } \\
1\end{array}$} & \multicolumn{2}{|c|}{$\begin{array}{l}\text { Fraction } \\
\text { Constituent }\end{array}$} & \multirow[b]{2}{*}{ Comments } \\
\hline & & & & & & 2 & 3 & & 2 & 3 & & 2 & 3 & \\
\hline 206-1-2 & $60-62$ & 2.1 & Unit $1 \mathrm{~A}$ & Pleist. & Calc. & & & \multirow{3}{*}{\multicolumn{3}{|c|}{$\begin{array}{l}\text { Insufficient residue } \\
\text { Quar. Mica Plag. } \\
\text { Insufficient residue } \\
\text { Quar. Mica Plag. }\end{array}$}} & Mica & Quar. & Kaol. & Mont. increases downhole, \\
\hline $206-10-2$ & $75-77$ & 82.3 & Calcareous ooze & & Calc. & & & & & & Mica & Quar. & Mont. & little volcanic glass \\
\hline \multirow{3}{*}{$\begin{array}{l}206-14-2 \\
206-15-3 \\
206-18-4\end{array}$} & \multirow{3}{*}{$\begin{array}{l}110-112 \\
110-112 \\
110-112\end{array}$} & \multirow{3}{*}{$\begin{array}{l}118.6 \\
129.1 \\
157.6\end{array}$} & \multirow{3}{*}{$\begin{array}{c}\text { Unit 1B } \\
\text { Calcareous ooze }\end{array}$} & \multirow{3}{*}{$\begin{array}{l}\text { Plio.- } \\
\text { Pleist. }\end{array}$} & Calc. & & & \multirow{2}{*}{\multicolumn{3}{|c|}{ Insufficient residue }} & \multirow{3}{*}{$\begin{array}{l}\text { Mont. } \\
\text { Mont. } \\
\text { Mont. }\end{array}$} & \multirow{3}{*}{$\begin{array}{l}\text { Quar. } \\
\text { Mica } \\
\text { Mica }\end{array}$} & \multirow{3}{*}{$\begin{array}{l}\text { Kaol. } \\
\text { Quar. } \\
\text { Quar. }\end{array}$} & \\
\hline & & & & & Calc. & & & & & & & & & \\
\hline & & & & & Calc. & & & & & & & & & \\
\hline $206-20-2$ & $60-62$ & 176.1 & \multirow{3}{*}{$\begin{array}{c}\text { Unit 1C } \\
\text { Calcareous ooze }\end{array}$} & M. Mio. to & Calc. & & & & & & Mica. & & & \\
\hline $206-22-3$ & $100-102$ & 196.0 & & E. Plio. & Calc. & & & Insuffic & cient res & idue & Mont. & Mica & Quar. & \\
\hline $\begin{array}{l}200-20-3 \\
206-31-3\end{array}$ & $\begin{array}{l}60-62 \\
60-62\end{array}$ & $\begin{array}{l}231.6 \\
280.6\end{array}$ & & & $\begin{array}{l}\text { Calc. } \\
\text { Calc. }\end{array}$ & & & & & & $\begin{array}{l}\text { Mont. } \\
\text { Mont. }\end{array}$ & $\begin{array}{l}\text { Kaol. } \\
\text { Quar. }\end{array}$ & $\begin{array}{l}\text { Quar. } \\
\text { Kaol. }\end{array}$ & \\
\hline $206-34-2$ & $30-32$ & 305.8 & D & E. to $\mathrm{M}$. & Calc. & & & Quar. & Plag. & & Mont. & Quar. & & \\
\hline $206-40-2$ & $60-62$ & 364.1 & $\begin{array}{l}\text { Argillaceous, cal- } \\
\text { careous ooze }\end{array}$ & Mio & Calc. & & & Quar. & Plag. & & Mont. & Quar. & Plag. & \\
\hline $206-44-1$ & $127-130$ & 399.3 & & & Calc. & Quar. & & Quar. & Plag. & Mica & Quar. & Mont. & & \\
\hline $206 \mathrm{C}-2-2$ & $49-51$ & 415.0 & & & Calc. & Quar. & & Quar. & Plag. & Mica & Quar. & Mont. & Mica & \\
\hline $206 \mathrm{C}-3-2$ & $60-62$ & 424.1 & & & Calc. & Quar. & & Quar. & Plag. & Mica & Quar. & Mont. & Plag. & \\
\hline $206 \mathrm{C}-4-2$ & $73-75$ & 433.2 & & & Calc. & Quar. & & Quar. & Plag. & Mica & Quar. & Mont. & Plag. & \\
\hline $206 \mathrm{C}-5-2$ & $83-86$ & 442.2 & Unit 2 & M. Olig. & Calc. & Quar. & & Quar. & Plag. & Mica & Quar. & Mont. & Plag. & Detrital quartz dominates \\
\hline $206 \mathrm{C}-6-3$ & $104-106$ & 466.0 & Lithified, semi- & & Calc. & Quar. & & Quar. & Plag. & Mica & Quar. & Mont. & Plag. & $2-20 \mu$ fraction, kaolinite \\
\hline $206 \mathrm{C}-7-1$ & $115-120$ & 481.1 & lithified clay & E. Mio. & Calc. & & & Quar. & Mont. & Plag. & Quar. & Mont. & Plag. & decreases downhole, little \\
\hline $206 \mathrm{C}-8-1$ & $58-60$ & 499.6 & nanno ooze & & Calc. & Quar. & & Quar. & Plag. & Mica & Mont. & Quar. & Mica & volcanic glass \\
\hline $206 \mathrm{C}-10-2$ & $125-128$ & 533.8 & & & Calc. & & & Quar. & Mont. & Plag. & Mont. & Quar. & & \\
\hline $206 C-11-5$ & $102-105$ & 554.0 & & & Calc. & Mont. & & Quar. & Mont. & Plag. & Mont. & Quar. & & \\
\hline $206 \mathrm{C}-12-2$ & $115-117$ & 567.7 & & & Calc. & & & Quar. & Plag. & & Mont. & Quar. & & \\
\hline $206 C-13-2$ & $87-92$ & 586.4 & & & Calc. & & & Quar. & Clin. & Plag. & Mont. & Quar. & & \\
\hline $206 C-3$ & $94-100$ & 587.9 & & & Calc. & & & Quar. & Clin. & Plag. & Mont. & Quar. & & \\
\hline 206C-14-2 & $92-95$ & 605.4 & & & Calc. & & & Quar. & Clin. & Plag. & Mont. & Quar. & & \\
\hline $\begin{array}{l}206 C-15-2 \\
206 C-16-1\end{array}$ & $\begin{array}{c}60-75 \\
141-142\end{array}$ & $\begin{array}{l}614.1 \\
631.4\end{array}$ & & & Calc. & & & Mont. & Plag. & Quar. & Mont. & Quar. & & \\
\hline $206 C-4$ & $\begin{array}{c}141-142 \\
60-75\end{array}$ & $\begin{array}{l}031.4 \\
635.1\end{array}$ & Radiolarian-rich & Eoc. & $\begin{array}{l}\text { Calc. } \\
\text { Calc. }\end{array}$ & & & $\begin{array}{l}\text { Mont. } \\
\text { Mont. }\end{array}$ & $\begin{array}{l}\text { Quar. } \\
\text { Ouar. }\end{array}$ & $\begin{array}{l}\text { Plag. } \\
\text { Plag. }\end{array}$ & $\begin{array}{l}\text { Mont. } \\
\text { Mont. }\end{array}$ & $\begin{array}{l}\text { Quar. } \\
\text { Quar. }\end{array}$ & & $\begin{array}{l}\text { Detrital quartz low in } \\
2-20 \mu \text { fraction }\end{array}$ \\
\hline $206 C-17-3$ & $65-83$ & 652.7 & calcareous ooze & & Calc. & & & Mont. & Quar. & Plag. & Mont. & & & \\
\hline $206 C-6$ & $120-122$ & 657.7 & & & Calc. & & & Mont. & Quar. & Plag. & Mont. & Quar. & Plag. & \\
\hline $206 \mathrm{C}-18-2$ & $119-139$ & 670.7 & & & Calc. & & & Mont. & Quar. & Plag. & Mont. & Quar. & & \\
\hline 206C-19-1 & $128-132$ & 688.3 & Unit 4 & M. Paleo. & Calc. & & & Bari. & Clin. & & Mont. & & & Barite present. \\
\hline $206 \mathrm{C}-20-1$ & $90-92$ & 706.9 & Lithified calcareous & to M. Eoc. & Calc. & & & Insuffic & cient res & idue & Mont. & Clin. & Mica & \\
\hline 206C-21-1 & $72-80$ & 725.7 & ooze and clay with & & Cris. & Calc. & & Cris. & Trid. & Quar. & Cris. & Mont. & Trid. & \\
\hline $206 \mathrm{C}-21-2$ & $78-92$ & 727.3 & subordinate chert & & Calc. & Cris. & & Cris. & Clin. & Trid. & Cris. & Trid. & Mont. & \\
\hline $207-3-4$ & $4-6$ & 19.5 & $\begin{array}{c}\text { Unit } 1 \\
\text { Calcareous ooze }\end{array}$ & E. Pleist. & Calc. & & & Insuffic & cient res & idue & Mica. & Quar. & Plag. & a \\
\hline $\begin{array}{l}207 \mathrm{~A}-33-2 \\
207 \mathrm{~A}-3\end{array}$ & $\begin{array}{c}4-6 \\
15-17\end{array}$ & $\begin{array}{l}349.5 \\
351.1\end{array}$ & $\begin{array}{l}\text { Unit } 3 \\
\text { Silty claystone }\end{array}$ & L. Cret. & $\begin{array}{l}\text { Cris. } \\
\text { Cris. }\end{array}$ & $\begin{array}{l}\text { Quar. } \\
\text { Trid. }\end{array}$ & Trid. & $\begin{array}{l}\text { Cris. } \\
\text { Cris. }\end{array}$ & $\begin{array}{l}\text { Quar. } \\
\text { Quar. }\end{array}$ & $\begin{array}{l}\text { Trid. } \\
\text { Plag. }\end{array}$ & $\begin{array}{l}\text { Cris. } \\
\text { Mont. }\end{array}$ & Mont. & & b \\
\hline
\end{tabular}


TABLE 2 - Continued

\begin{tabular}{|c|c|c|c|c|c|c|c|c|c|c|c|c|c|c|}
\hline \multirow[b]{2}{*}{ Sample } & \multirow{2}{*}{$\begin{array}{c}\text { Sample } \\
\text { Depth in } \\
\text { Section }(\mathrm{cm})\end{array}$} & \multirow{2}{*}{$\begin{array}{l}\text { Sample } \\
\text { Depth Below } \\
\text { Sea Floor (m) }\end{array}$} & \multirow[b]{2}{*}{ Lithology } & \multirow[b]{2}{*}{ Age } & \multirow{2}{*}{$\begin{array}{l}\text { Bulk } \\
\text { Major } \\
1\end{array}$} & \multicolumn{2}{|c|}{$\begin{array}{c}\text { Sample } \\
\text { Constituent }\end{array}$} & \multirow{2}{*}{$\begin{array}{l}2-20 \mu \\
\text { Major } \\
1\end{array}$} & \multicolumn{2}{|c|}{$\begin{array}{l}\text { Fraction } \\
\text { Constituent }\end{array}$} & \multirow{2}{*}{$\begin{array}{l}<2 \mu \\
\text { Major } \\
\quad 1\end{array}$} & \multicolumn{2}{|c|}{$\begin{array}{c}\text { Fraction } \\
\text { Constituent }\end{array}$} & \multirow[b]{2}{*}{ Comments } \\
\hline & & & & & & 2 & 3 & & 2 & 3 & & 2 & 3 & \\
\hline $\begin{array}{l}207 \mathrm{~A}-42-2 \\
207 \mathrm{~A}-46-3 \\
\end{array}$ & $\begin{array}{l}96-98 \\
70-71 \\
\end{array}$ & $\begin{array}{l}434.5 \\
471.7 \\
\end{array}$ & $\begin{array}{l}\text { Unit } 5 \\
\text { Vitrophyric rhyolite }\end{array}$ & L. Cret. & $\begin{array}{l}\text { Mont. } \\
\text { Cris. }\end{array}$ & Mont. & Plag. & $\begin{array}{l}\text { Mont. } \\
\text { Mont. }\end{array}$ & $\begin{array}{l}\text { Cris. } \\
\text { Cris. }\end{array}$ & $\begin{array}{l}\text { Plag. } \\
\text { Plag. }\end{array}$ & $\begin{array}{l}\text { Mont. } \\
\text { Mont. }\end{array}$ & & & b \\
\hline $\begin{array}{l}208-30-3 \\
208-33-3 \\
208-34-3\end{array}$ & $\begin{array}{c}90-92 \\
89-91 \\
127-140\end{array}$ & $\begin{array}{l}551.9 \\
579.9 \\
589.3\end{array}$ & $\begin{array}{l}\text { Unit } 2 \\
\text { Siliceous and cal- } \\
\text { careous ooze }\end{array}$ & $\begin{array}{l}\text { L. Cret. to } \\
\text { M. Eoc. }\end{array}$ & $\begin{array}{l}\text { Calc. } \\
\text { Calc. } \\
\text { Calc. }\end{array}$ & $\begin{array}{l}\text { Cris. } \\
\text { Cris. }\end{array}$ & & $\begin{array}{l}\text { Mont. } \\
\text { Cris. } \\
\text { Clin. }\end{array}$ & $\begin{array}{l}\text { Bari. } \\
\text { Clin. } \\
\text { Quar. }\end{array}$ & $\begin{array}{l}\text { Quar. } \\
\text { Quar. } \\
\text { Cris. }\end{array}$ & $\begin{array}{l}\text { Mont. } \\
\text { Cris. } \\
\text { Cris. }\end{array}$ & $\begin{array}{l}\text { Bari. } \\
\text { Trid. } \\
\text { Mont. }\end{array}$ & $\begin{array}{l}\text { Quar. } \\
\text { Mont. } \\
\text { Trid. }\end{array}$ & Barite present \\
\hline
\end{tabular}

aKaolinite and chlorite are present as at Site 206.

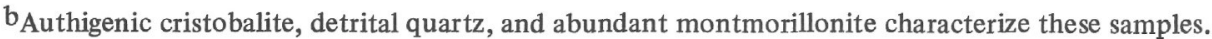

TABLE 3

Summary of X-ray Mineralogy Samples, Sample Depths, Lithology, Age, and X-ray Diffraction Results, Site 209

\begin{tabular}{|c|c|c|c|c|c|c|c|c|c|c|c|c|c|c|}
\hline \multirow[b]{2}{*}{ Sample } & \multirow{2}{*}{$\begin{array}{c}\text { Sample } \\
\text { Depth in } \\
\text { Section }(\mathrm{cm})\end{array}$} & \multirow{2}{*}{$\begin{array}{c}\text { Sample } \\
\text { Depth Below } \\
\text { Sea Floor }(\mathrm{m})\end{array}$} & \multirow[b]{2}{*}{ Lithology } & \multirow[b]{2}{*}{ Age } & \multirow{2}{*}{$\begin{array}{l}\text { Bulk } \\
\text { Major } \\
\quad 1\end{array}$} & \multicolumn{2}{|c|}{$\begin{array}{c}\text { Sample } \\
\text { Constituent }\end{array}$} & \multirow{2}{*}{$\begin{array}{l}2-20 \mu \\
\text { Major } \\
\quad 1\end{array}$} & \multicolumn{2}{|c|}{$\begin{array}{l}\text { Fraction } \\
\text { Constituent }\end{array}$} & \multirow{2}{*}{$\begin{array}{l}<2 \mu \\
\text { Major } \\
1\end{array}$} & \multicolumn{2}{|c|}{$\begin{array}{l}\text { Fraction } \\
\text { Constituent }\end{array}$} & \multirow[b]{2}{*}{ Comments } \\
\hline & & & & & & 2 & 3 & & 2 & 3 & & 2 & 3 & \\
\hline $209-1-4$ & $110-112$ & 5.6 & \multirow{8}{*}{$\begin{array}{l}\text { Unit } 1 \\
\text { Foram and nanno } \\
\text { ooze }\end{array}$} & \multirow{8}{*}{$\begin{array}{l}\text { L. Olig to } \\
\text { Pleist. }\end{array}$} & Calc. & & & \multirow{8}{*}{\multicolumn{3}{|c|}{$\begin{array}{l}\text { Insufficient residue } \\
\text { after decalcification }\end{array}$}} & Mont. & Mica & Kaol. & \multirow{8}{*}{$\mathrm{a}, \mathrm{b}$} \\
\hline $209-2-4$ & $116-118$ & 14.7 & & & Calc. & & & & & & Mont. & Plag. & Kaol. & \\
\hline $209-3-4$ & $116-118$ & 23.7 & & & Calc. & & & & & & Mont. & Mica & Kaol. & \\
\hline $209-4-4$ & $116-118$ & 32.7 & & & Calc. & & & & & & Mont. & Kaol. & Mica. & \\
\hline $209-5-4$ & $116-118$ & 41.7 & & & Calc. & & & & & & Mont. & Kaol. & Mica. & \\
\hline 209-6-5 & $108-118$ & 52.2 & & & Calc. & & & & & & Mont. & Kaol. & Quar. & \\
\hline 209-9-1 & $130-132$ & 75.3 & & & Calc. & & & & & & Insuffi & cient res & idue & \\
\hline $209-14-2$ & $116-118$ & 133.7 & & & Calc. & & & & & & Apat. & Mont. & Plag. & \\
\hline $209-15-1$ & $100-102$ & 151.0 & \multirow{5}{*}{$\begin{array}{l}\text { Unit } 2 \\
\text { Sandy foram ooze } \\
\text { and chert }\end{array}$} & \multirow{5}{*}{ L. Eoc. } & Calc. & & & \multicolumn{3}{|c|}{ Insufficient residue } & Mont. & Cris. & Apat. & \multirow{5}{*}{$a, b, c, d$} \\
\hline $209-23-2$ & $110-112$ & 243.6 & & & Calc. & Qua & & Quar. & $\mathrm{K}-\mathrm{Fe}$. & Plag. & Mont. & Kaol. & Mica & \\
\hline $209-27-2$ & $64-66$ & 274.1 & & & Calc. & Cris. & & Cris. & Trid. & Quar. & Cris. & Trid. & Mont. & \\
\hline $209-28-2$ & $78-86$ & 283.3 & & & Calc. & Cris. & Quar. & Cris. & Trid. & Quar. & Cris. & Trid. & Mont. & \\
\hline $209-29-1$ & $135-140$ & 291.3 & & & Calc. & Quar. & Plag. & Quar. & Plag. & Clin. & Mont. & Cris. & Trid. & \\
\hline $209-31-1$ & $122-130$ & 309.2 & Unit 3 & \multirow{3}{*}{ M. Eoc. } & Calc. & Cris. & Quar. & Cris. & Quar. & Plag. & Cris. & Trid. & Mont. & \multirow{3}{*}{$a, b, c, d$} \\
\hline $209-32-1$ & $125-140$ & 318.3 & Calcareous silty & & Calc. & Cris. & Quar. & Cris. & Quar. & Plag. & Cris. & Mont. & Trid. & \\
\hline $209-34-2$ & $96-106$ & 337.5 & $\begin{array}{l}\text { sandstone and } \\
\text { sandy siltstone }\end{array}$ & & Calc. & & & Cris. & Quar. & Plag. & Cris. & Trid. & Mont. & \\
\hline
\end{tabular}

aDetrital quartz grains with variable amounts of inclusions.

$\mathrm{b}_{\text {Few }}$ altered volcanic shards present.

cApatite present.

d Kaolinite not detected. 
TABLE 4

Summary of X-ray Mineralogy Samples, Sample Depths, Lithology, Age, and X-ray Diffraction Results,

Site 210

\begin{tabular}{|c|c|c|c|c|c|c|c|c|c|c|c|c|c|c|}
\hline \multirow[b]{2}{*}{ Sample } & \multirow{2}{*}{$\begin{array}{c}\text { Sample } \\
\text { Depth in } \\
\text { Section }(\mathrm{cm})\end{array}$} & \multirow{2}{*}{$\begin{array}{c}\text { Sample } \\
\text { Depth Below } \\
\text { Sea Floor (m) }\end{array}$} & \multirow[b]{2}{*}{ Lithology } & \multirow[b]{2}{*}{ Agw } & \multirow{2}{*}{$\begin{array}{l}\text { Bulk } \\
\text { Major } \\
\quad 1\end{array}$} & \multicolumn{2}{|c|}{$\begin{array}{l}\text { Sample } \\
\text { Constituents }\end{array}$} & \multirow{2}{*}{$\begin{array}{l}2-20 \mu \\
\text { Major } \\
\quad 1\end{array}$} & \multicolumn{2}{|c|}{$\begin{array}{l}\text { Fraction } \\
\text { Constituents }\end{array}$} & \multirow{2}{*}{$\begin{array}{l}<2 \mu \\
\text { Major } \\
1\end{array}$} & \multicolumn{2}{|c|}{$\begin{array}{l}\text { Fraction } \\
\text { Constituents }\end{array}$} & \multirow[b]{2}{*}{ Comments } \\
\hline & & & & & & 2 & 3 & & 2 & 3 & & 2 & 3 & \\
\hline 210-1-1 & $32-34$ & 0.03 & \multirow{36}{*}{ Unit 1} & \multirow{36}{*}{$\begin{array}{l}\text { L. Miocene } \\
\text { through L. } \\
\text { Pleistocene }\end{array}$} & Calc. & Mica & Quar. & Quar. & Plag. & Mica & Mont. & Mica & Quar. & \multirow{36}{*}{$\begin{array}{l}\text { Chlorite and amphibole } \\
\text { present throughout. Kaolinite } \\
\text { sporadic below Core } 23 \text {. } \\
\text { Aragonite may be } \\
\text { allochthanous. a }\end{array}$} \\
\hline $210-4$ & $22-24$ & 4.7 & & & Mica & Quar. & Plag. & Quar. & Mica & Plag. & Mica & Mont. & Quar. & \\
\hline $210-6$ & $125-127$ & 8.8 & & & Quar. & Mica. & Plag. & Plag. & & & Mont. & Mica & Quar. & \\
\hline $210-2-1$ & $41-43$ & 9.4 & & & Quar. & Plag. & Mica. & Quar. & Plag. & Mica & Mica & Mont. & Quar. & \\
\hline $210-3$ & $78-80$ & 12.8 & & & Quar. & Plag. & Mica & Quar. & Plag. & Mica & Mica & Mont. & Quar. & \\
\hline $210-6$ & $25-27$ & 16.8 & & & Quar. & Mica & Plag. & Quar. & Plag. & Mica & Mont. & Mica & Quar. & \\
\hline $210-6$ & $46-48$ & I7.0 & & & Mica & Quar. & Plag. & Quar. & Mica & Plag. & Mont. & Mica & Quar. & \\
\hline $210-3-3$ & $13-15$ & 21.1 & & & Quar. & Plag. & Mica & Quar. & Plag. & Mica & Mica & Mont. & Quar. & \\
\hline $210-4$ & $70-72$ & 23.2 & & & Calc. & Arag. & Quar. & Quar. & Plag. & Mica & Mont. & Mica & Kaol. & \\
\hline $210-6-3$ & $143-145$ & 49.4 & & & Calc. & Arag. & Quar. & Quar. & Mica & Plag. & Kaol. & Mica & Mont. & \\
\hline $210-8-5$ & $65-67$ & 69.7 & & & Mica. & Quar. & Plag. & Mica & Quar. & Plag. & Mont. & Mica & Quar. & \\
\hline $210-5$ & $116-118$ & 70.2 & & & Arag. & Calc. & Quar. & Quar. & Plag. & Mica & Mont. & Mica & Kaol, & \\
\hline $210-9-2$ & $26-28$ & 73.8 & & & Calc. & Arag. & & Quar. & Plag. & Mica & Mica & Quar. & Plag. & \\
\hline $\begin{array}{l}210-10-4 \\
210-4\end{array}$ & $\begin{array}{c}48-50 \\
113-115\end{array}$ & $\begin{array}{l}86.0 \\
86.6\end{array}$ & & & Quar. & $\begin{array}{l}\text { Mica } \\
\text { Calc. }\end{array}$ & Plag. & Quar. & $\begin{array}{l}\text { Mica } \\
\text {. }\end{array}$ & Plag. & Mont. & Mica & Quar. & \\
\hline $210-11-2$ & $20-22$ & $\begin{array}{r}80.0 \\
104.7\end{array}$ & & & Arag. & Calc. & Ouar: & \multirow{2}{*}{\multicolumn{3}{|c|}{$\begin{array}{l}\text { Insufficient residue } \\
\text { Quar. Plag. }\end{array}$}} & \multicolumn{3}{|c|}{ Insufficient residue } & \\
\hline $210-2$ & $63-65$ & 105.1 & & & $\begin{array}{l}\text { Arag. } \\
\text { Plag. }\end{array}$ & $\begin{array}{l}\text { Calc. } \\
\text { Ouar. }\end{array}$ & $\begin{array}{l}\text { Quar. } \\
\text { Mica }\end{array}$ & $\begin{array}{l}\text { Quar. } \\
\text { Plag. }\end{array}$ & & & Mont. & Mica & Kaol. & \\
\hline $210-12-3$ & $112.5-114.5$ & 126.1 & & & Mica & Quar. & Plag. & Quar. & $\begin{array}{l}\text { Quar. } \\
\text { Mica. }\end{array}$ & $\begin{array}{l}\text { Mica } \\
\text { Plag. }\end{array}$ & $\begin{array}{l}\text { Mont. } \\
\text { Mont. }\end{array}$ & Mica & Quar. & \\
\hline $210-20-1$ & $128-130$ & 273.3 & & & Quar. & Plag. & Mica & Mica & Quar. & Plag. & Mont. & Mica & Quar. & \\
\hline $210-21-1$ & $72-74$ & 291.7 & & & Calc. & & & Quar. & Plag. & Mica & Mont. & Quar. & Mica & \\
\hline $210-2$ & $53-55$ & 293.0 & & & Calc. & Quar. & Plag. & Mica & Quar. & Plag. & Mont. & Mica & Quar. & \\
\hline $210-22-2$ & 93-95 & 311.4 & & & Calc. & Arag. & Mica & Quar. & Plag. & Mica & Mont. & Quar. & Mica & \\
\hline $210-5$ & $8-10$ & 315.1 & & & Mica & Quar. & Plag. & Quar. & Plag. & Mica & Mont. & Kaol. & Quar. & \\
\hline $210-5$ & $27.5-29.5$ & 315.3 & & & Mica & Quar. & Plag. & Mica & Quar. & Plag. & Mont. & Mica & Quar. & \\
\hline $210-5$ & $55-57$ & 315.5 & & & Calc. & Mica & & Quar. & Mica & Plag. & Mont. & Kaol. & Mica & \\
\hline 210-23-1 & $130-132$ & 329.3 & & & Calc. & Arag. & Plag. & Quar. & Plag. & Mica & Mont. & Kaol. & Quar. & \\
\hline $210-4$ & $147-149$ & 334.0 & & & Plag. & Quar. & Mica & Plag. & Quar. & Mica & Mont. & Mica & Quar. & \\
\hline $210-24-2$ & $108-110$ & 349.6 & & & Calc. & Mica & Quar. & Mica & Quar. & Plag. & Mont. & Mica & Quar. & \\
\hline $210-6$ & $131-133$ & 355.8 & & & Calc. & Plag. & Quar. & Chlo. & Plag. & Quar. & Mont. & Phil. & & \\
\hline $210-25-1$ & $78-81$ & 365.8 & & & Calc. & Mont. & & Insuffic & cient res & sidue & Mont. & Phil. & & \\
\hline $210-3$ & $22-24$ & 368.2 & & & Quar. & Plag. & Mica & Mica & Quar. & Plag. & Mica & Mont. & Quar. & \\
\hline $210-4$ & $113-115$ & 370.6 & & & Mica & Plag. & Quar. & Plag. & Quar. & Mica & Mont. & Mica & & \\
\hline $210-27-3$ & $108.5-110.5$ & 407.1 & & & Quar. & Plag. & Mica & Quar. & Plag. & Mica & Mont. & Mica & Quar. & \\
\hline $210-6$ & $138-140$ & 411.9 & & & Quar. & Plag. & Mica & Quar. & Plag. & Mica & Mont. & Quar. & Mica & \\
\hline $210-28-6$ & $96-98$ & 429.5 & & & Mica & Quar. & Plag. & Mica & Quar. & Plag. & Mica & Mont. & Chlo. & \\
\hline $210-29-3$ & $87-89$ & 443.9 & & & Mica & Plag. & Quar. & Mica & Plag. & Quar. & Mont. & Mica & Quar. & \\
\hline $210-30-3$ & 76-78 & 462.8 & & & Quar. & Plag. & Mica & Mica & Quar. & Plag. & Mont. & Mica & Quar. & \\
\hline $210-31-3$ & $9-13$ & 480.1 & Unit 2 & E. and $\mathrm{M}$. & Mica & Plag. & Quar. & Mica & Plag. & Quar. & Mica & Mont. & Plag. & \\
\hline $210-32-6$ & $24-26$ & 503.7 & $\begin{array}{l}\text { Silty clay (with no } \\
\text { no graded cycles) }\end{array}$ & Mio. & Mont. & Quar. & Mica. & Quar. & $\mathrm{K}-\mathrm{Fe}$. & Plag. & Mont. & Mica & Kaol. & $a, c$ \\
\hline $210-34-1$ & $72-74$ & 533.7 & $\begin{array}{l}\text { Unit } 3 \\
\text { Argillaceous chalk }\end{array}$ & $\begin{array}{l}\text { E.-M. } \\
\text { Olig. }\end{array}$ & \multicolumn{3}{|l|}{ Calc. } & \multicolumn{3}{|c|}{ Insufficient residue } & Mont. & Clin. & & $\mathrm{a}$ \\
\hline $210-35-4$ & $116-118$ & 547.7 & Unit 4 & \multirow[t]{2}{*}{ L. Eoc. } & Calc & & & Insuffic & cient re & sidue & Mont. & & & $\mathrm{b}$ \\
\hline $210-36-1$ & $70-72$ & 551.7 & Argillaceous chalk & & Calc. & & & Quar. & Clin. & Mica & Mont. & Mica & & \\
\hline
\end{tabular}


TABLE 4 - Continued

\begin{tabular}{|c|c|c|c|c|c|c|c|c|c|c|c|c|c|}
\hline \multirow[b]{3}{*}{ Sample } & \multirow{3}{*}{$\begin{array}{c}\text { Sample } \\
\text { Depth in } \\
\text { Section }(\mathrm{cm}) \\
\end{array}$} & \multirow{3}{*}{$\begin{array}{l}\text { Sample } \\
\text { Depth Below } \\
\text { Sea Floor (m) }\end{array}$} & \multirow[b]{3}{*}{ Lithology } & \multirow[b]{3}{*}{ Age } & \multicolumn{2}{|c|}{ Bulk Sample } & \multicolumn{3}{|c|}{$2-20 \mu$ Fraction } & \multicolumn{3}{|c|}{$<2 \mu$ Fraction } & \multirow[b]{3}{*}{ Comments } \\
\hline & & & & & Major & Constituents & Major & Constit & uents & Major & Constitu & lents & \\
\hline & & & & & 1 & 2 & 1 & 2 & 3 & 1 & 2 & 3 & \\
\hline $210-36-4$ & $34-34$ & 555.8 & \multirow{15}{*}{ Unit 5} & \multirow{15}{*}{$\begin{array}{l}\text { E. Eocene } \\
\text { through } \\
\text { M. Eocene }\end{array}$} & Calc. & & Quar. & Mica & Clin. & Mont. & Cris. & Mica & \\
\hline $210-37-3$ & $99-100$ & 564.0 & & & Calc. & & Quar. & Clin. & Mica & Mont. & Mica & Quar. & \\
\hline $210-38-2$ & $81-82$ & 571.3 & & & Calc. & & & & & Mont. & Cris. & Mica & \\
\hline $210-39-2$ & $29-30$ & 579.8 & & & Calc. & Cris. & Cris. & Quar. & Mica & Cris. & Mont. & Trid. & \\
\hline $210-40-3$ & $42-44$ & 590.4 & & & Calc. & Cris. & Cris. & Quar. & Mica & Cris. & Mont. & Trid. & \\
\hline $210-41-2$ & $0-2$ & 597.5 & & & Calc. & Cris. & Cris. & Quar. & Mica & Cris. & Mont. & Quar. & \\
\hline $210-42-1$ & $59-61$ & 605.6 & & & Calc. & Cris. & Quar. & Cris. & Mica & Cris. & Mont. & Quar. & $\mathrm{a}$ \\
\hline $210-43-4$ & $88-89$ & 619.4 & & & Calc. & Quar. & Quar. & Mica & & Quar. & Mont. & & \\
\hline $210-44-2$ & $96-97$ & 625.5 & & & Calc. & Quar. & Quar. & Mica & & Quar. & Mont. & & \\
\hline $210-45-2$ & $104-105$ & 634.5 & & & Calc. & Quar. & Quar. & Mica & & Quar. & Mont. & & \\
\hline $210-46-4$ & $62-64$ & 653.1 & & & Calc. & Quar. & Quar. & Mica & & Quar. & Mont. & & \\
\hline $210-47-3$ & $28-30$ & 668.3 & & & Calc. & Quar. & Quar. & Mica. & Plag. & Quar. & Mont. & & \\
\hline $210-48-2$ & $52-54$ & 680.0 & & & Calc. & Quar. & Insuffi & cient res & idue & Quar. & Mont. & Mica. & \\
\hline $210-49-2$ & $80-83$ & 694.3 & & & Calc. & Quar. & Quar. & Plag. & & Quar. & Mont. & Mica & \\
\hline $210-50-4$ & $128-130$ & $707: 8$ & & & Calc. & Quar. & Quar. & Mica & Plag. & Quar. & Mica & Chlo. & \\
\hline
\end{tabular}

Similar mineral suite as base of Unit 1, but turbidites absent.

bMineralogy similar to Unit 5 but no authigenic silicates were detected.

cFew, altered volcanic glass shards present. Detrital quartz grains commonly contain inclusions. 
TABLE 5

Results of X-Ray Diffraction Analyses, Hole 203

\begin{tabular}{|c|c|c|c|c|c|c|c|c|c|c|c|c|}
\hline Core & $\begin{array}{c}\text { Cored } \\
\text { Interval } \\
\text { Below Sea } \\
\text { Floor (m) }\end{array}$ & $\begin{array}{l}\text { Sample Deptha } \\
\text { Below Sea } \\
\text { Floor } \\
\text { (m) }\end{array}$ & 出 & 㻤 & $\frac{\dot{0}}{\sqrt{0}}$ & 岂 & $\frac{\text { bj }}{\frac{\pi}{2}}$ & $\frac{\dot{0}}{\text { 己 }}$ & 范 & . & $\stackrel{\overrightarrow{0}}{\sum_{4}^{\circ}}$ & $\begin{array}{l}\dot{0}_{0} \\
\sum^{\leftrightarrows}\end{array}$ \\
\hline \multicolumn{13}{|c|}{ Bulk Samples } \\
\hline 3 & $108-117$ & 113.7 & 97.6 & 96.3 & 44.8 & 4.9 & 35.5 & 3.8 & 10.9 & & - & - \\
\hline 4 & $229-238$ & 230.1 & 84.6 & 75.9 & 25.4 & 7.1 & 46.1 & 4.3 & 5.9 & & 7.6 & 3.6 \\
\hline \multicolumn{13}{|c|}{$2-20 \mu$ Fraction } \\
\hline 3 & $108-117$ & 113.7 & 98.9 & 98.3 & & 13.3 & 86.7 & - & & & - & - \\
\hline 4 & $229-238$ & 230.1 & 80.7 & 69.8 & & 11.5 & 59.5 & 4.1 & & & 21.3 & 3.6 \\
\hline \multicolumn{13}{|c|}{$<2 \mu$ Fraction } \\
\hline 3 & $108-117$ & 113.7 & 92.3 & 87.9 & - & 3.9 & 27.9 & 14.4 & 53.9 & - & - & - \\
\hline 4 & $229-238$ & 230.1 & 81.5 & 71.0 & - & 1.8 & 21.3 & 8.7 & 56.6 & 1.8 & 8.6 & 1.2 \\
\hline
\end{tabular}


TABLE 6

Results of X-ray Diffraction Analyses from Hole 204

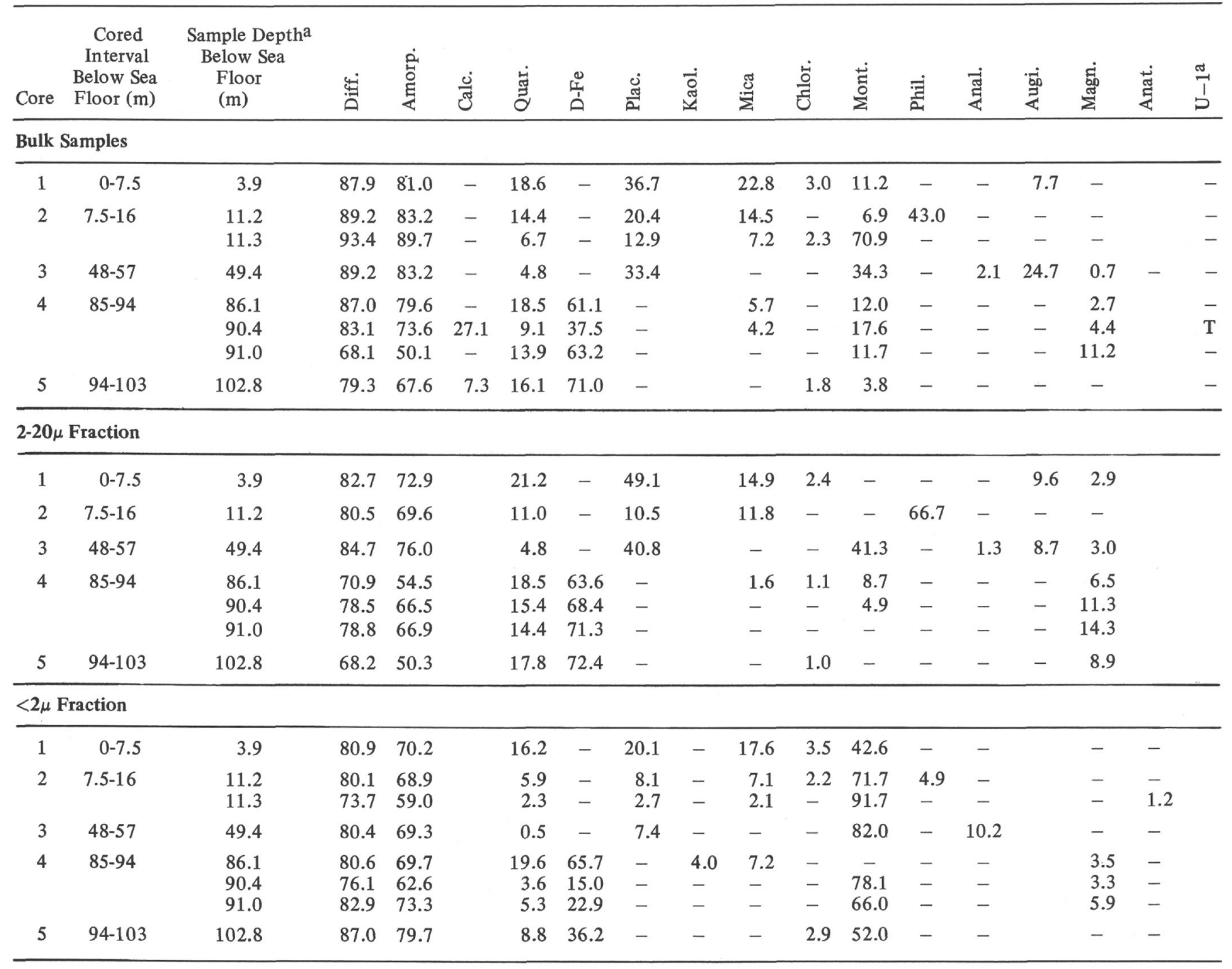

aPeaks at $12.1 \AA . \mathrm{T}=$ trace. 
TABLE 7

Results of X-ray Diffraction Analyses from Hole 205

\begin{tabular}{|c|c|c|c|c|c|c|c|c|c|c|c|c|c|c|c|c|c|c|c|c|}
\hline Core & $\begin{array}{c}\text { Cored } \\
\text { Interval } \\
\text { Below Sea } \\
\text { Floor }(\mathrm{m})\end{array}$ & $\begin{array}{l}\text { Sample Deptha } \\
\text { Below Sea } \\
\text { Floor } \\
\text { (m) }\end{array}$ & 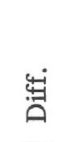 & $\begin{array}{l}\text { 它 } \\
\text { 完 }\end{array}$ & $\frac{\dot{0}}{\text { हुँ }}$ & 莺 & 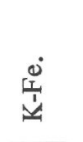 & 寻 & 㲾 & $\stackrel{\overparen{ٌ}}{\Sigma}$ & 㝘 & 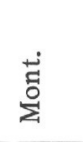 & . & 音 & 丞 & 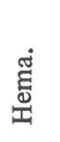 & 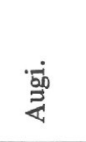 & 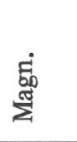 & 巡 & $\stackrel{\sigma}{\vec{b}}$ \\
\hline \multicolumn{21}{|c|}{ Bulk Samples } \\
\hline 2 & $9-18$ & $\begin{array}{l}10.1 \\
14.3\end{array}$ & $\begin{array}{l}90.1 \\
91.2\end{array}$ & $\begin{array}{l}84.5 \\
86.3\end{array}$ & $\overline{1.7}$ & $\begin{array}{r}11.6 \\
9.4\end{array}$ & $\begin{array}{l}- \\
-\end{array}$ & $\begin{array}{l}46.3 \\
39.8\end{array}$ & & $\begin{array}{r}7.0 \\
12.8\end{array}$ & $\begin{array}{l}- \\
-\end{array}$ & $\begin{array}{r}11.9 \\
3.7\end{array}$ & $\begin{array}{l}- \\
-\end{array}$ & $\begin{array}{r}5.5 \\
23.2\end{array}$ & $\begin{array}{l}0.9 \\
1.1\end{array}$ & & $\begin{array}{r}11.3 \\
3.8\end{array}$ & $\begin{array}{l}5.4 \\
4.5\end{array}$ & $\begin{array}{l}- \\
-\end{array}$ & $\begin{array}{l}- \\
-\end{array}$ \\
\hline 3 & $18-27$ & 27.0 & 79.3 & 67.7 & - & 2.5 & - & 31.7 & & - & - & 12.5 & - & 48.5 & - & & - & 4.8 & - & - \\
\hline 5 & $38-47$ & 42.1 & 93.0 & 89.0 & 63.4 & 1.8 & 12.4 & 16.1 & & - & - & - & - & - & - & & - & 6.3 & - & - \\
\hline 7 & $56-65$ & 56.5 & 76.3 & 63.0 & 60.3 & 2.0 & - & 26.4 & & - & - & - & - & - & - & & 9.4 & 2.0 & - & - \\
\hline 8 & $65-74$ & $\begin{array}{l}67.4 \\
69.0 \\
74.0\end{array}$ & $\begin{array}{l}90.9 \\
84.2 \\
79.7\end{array}$ & $\begin{array}{l}85.9 \\
75.3 \\
68.3\end{array}$ & $\begin{array}{r}34.4 \\
15.5 \\
8.4\end{array}$ & $\begin{array}{l}5.8 \\
4.6 \\
5.2\end{array}$ & $\begin{array}{l}- \\
- \\
-\end{array}$ & $\begin{array}{l}41.8 \\
56.6 \\
62.9\end{array}$ & & $\begin{array}{l}- \\
-\end{array}$ & $\begin{array}{l}\overline{3.3} \\
-\end{array}$ & $\begin{array}{l}5.8 \\
- \\
2.7\end{array}$ & $\begin{array}{l}- \\
- \\
-\end{array}$ & $\begin{array}{l}- \\
- \\
-\end{array}$ & $\begin{array}{l}- \\
- \\
-\end{array}$ & & $\begin{array}{r}7.9 \\
15.0 \\
16.7\end{array}$ & $\begin{array}{l}4.3 \\
5.0 \\
4.1\end{array}$ & $\begin{array}{l}- \\
- \\
-\end{array}$ & $\begin{array}{l}- \\
- \\
-\end{array}$ \\
\hline 11 & $95-104$ & 97.6 & 89.8 & 84.0 & 18.3 & 5.6 & - & 38.2 & & - & - & 12.5 & 2.1 & 5.4 & - & & 12.8 & 5.1 & - & - \\
\hline 12 & $104-113$ & 113.0 & 91.7 & 87.1 & 8.6 & 3.8 & - & 49.9 & & - & - & 11.4 & - & - & 0.9 & & 20.5 & 4.9 & - & - \\
\hline 15 & $131-140$ & $\begin{array}{l}133.6 \\
134.8 \\
140.0\end{array}$ & $\begin{array}{l}92.7 \\
99.5 \\
91.8\end{array}$ & $\begin{array}{l}88.6 \\
99.2 \\
87.5\end{array}$ & $\begin{array}{l}7.2 \\
\overline{4} .0\end{array}$ & $\begin{array}{l}6.9 \\
8.0 \\
7.9\end{array}$ & $\begin{array}{l}- \\
- \\
-\end{array}$ & $\begin{array}{c}45.8 \\
35.5 \\
-\end{array}$ & & $\begin{array}{l}- \\
- \\
-\end{array}$ & $\begin{array}{l}- \\
- \\
-\end{array}$ & $\begin{array}{l}21.8 \\
36.7 \\
31.3\end{array}$ & $\begin{array}{l}- \\
- \\
-\end{array}$ & $\begin{array}{l}- \\
- \\
-\end{array}$ & $\begin{array}{l}2.0 \\
\overline{5.1}\end{array}$ & & $\begin{array}{l}11.9 \\
17.8 \\
45.4\end{array}$ & $\begin{array}{l}4.3 \\
2.1 \\
6.3\end{array}$ & $\begin{array}{l}- \\
- \\
-\end{array}$ & $\begin{array}{l}- \\
-\end{array}$ \\
\hline 16 & $142-151$ & 145.8 & 91.9 & 87.4 & 29.1 & 4.1 & - & 47.3 & & - & - & 7.0 & - & - & - & & 6.9 & 5.6 & - & $\mathrm{T}$ \\
\hline 17 & $151-160$ & 155.0 & 94.3 & 91.2 & 11.0 & 8.0 & - & 38.6 & & - & - & 23.7 & - & - & 2.6 & & 12.9 & 3.2 & - & - \\
\hline 23 & $246-255$ & 255.0 & 82.9 & 73.3 & 30.0 & 2.3 & - & 42.6 & & - & - & 23.8 & - & - & - & & - & 1.4 & - & - \\
\hline 24 & $274-283$ & $\begin{array}{l}277.7 \\
283.0\end{array}$ & $\begin{array}{l}71.9 \\
64.2\end{array}$ & $\begin{array}{l}56.0 \\
44.1\end{array}$ & $\begin{array}{l}69.7 \\
90.7\end{array}$ & $\begin{array}{l}2.3 \\
1.9\end{array}$ & $\begin{array}{l}- \\
-\end{array}$ & $\begin{array}{r}12.3 \\
5.5\end{array}$ & & - & $\begin{array}{l}- \\
-\end{array}$ & $\begin{array}{r}14.0 \\
2.0\end{array}$ & $\begin{array}{l}1.8 \\
-\end{array}$ & - & - & & - & - & - & - \\
\hline 25 & 283-292 & 292.0 & 71.9 & 56.1 & 55.6 & 1.4 & - & 25.5 & & - & - & 17.5 & - & - & - & & - & - & - & - \\
\hline 26 & 292-301 & $\begin{array}{l}295.6 \\
301.0\end{array}$ & $\begin{array}{l}73.2 \\
81.3\end{array}$ & $\begin{array}{l}58.1 \\
70.9\end{array}$ & $\begin{array}{l}69.9 \\
25.0\end{array}$ & $\begin{array}{l}4.2 \\
2.4\end{array}$ & $\begin{array}{l}- \\
-\end{array}$ & $\begin{array}{l}7.3 \\
9.6\end{array}$ & & $\begin{array}{l}2.3 \\
6.1\end{array}$ & $\begin{array}{l}- \\
-\end{array}$ & $\begin{array}{l}15.1 \\
56.9\end{array}$ & $\begin{array}{l}1.1 \\
-\end{array}$ & $\begin{array}{l}- \\
-\end{array}$ & $\begin{array}{l}- \\
-\end{array}$ & & $\begin{array}{l}- \\
-\end{array}$ & $\begin{array}{l}- \\
-\end{array}$ & $\begin{array}{l}- \\
-\end{array}$ & - \\
\hline 27 & $301-310$ & 310.0 & 76.7 & 63.7 & 80.0 & 2.9 & - & 5.3 & & 3.0 & - & 8.9 & - & - & - & & - & - & - & - \\
\hline 28 & 310-319 & $\begin{array}{l}311.0 \\
311.4 \\
319.0\end{array}$ & $\begin{array}{l}81.4 \\
81.6 \\
72.1\end{array}$ & $\begin{array}{l}71.0 \\
71.2 \\
56.4\end{array}$ & $\begin{array}{r}2.2 \\
26.1 \\
89.3\end{array}$ & $\begin{array}{l}3.6 \\
1.6 \\
3.4\end{array}$ & $\begin{array}{l}- \\
- \\
-\end{array}$ & $\begin{array}{c}35.6 \\
7.9 \\
-\end{array}$ & & $\begin{array}{c}12.8 \\
- \\
-\end{array}$ & $\begin{array}{l}- \\
- \\
-\end{array}$ & $\begin{array}{c}45.7 \\
64.3 \\
-\end{array}$ & $\begin{array}{l}- \\
- \\
2.7\end{array}$ & $\begin{array}{l}- \\
- \\
4.7\end{array}$ & $\begin{array}{l}- \\
- \\
-\end{array}$ & & $\begin{array}{l}- \\
- \\
-\end{array}$ & $\begin{array}{l}- \\
- \\
-\end{array}$ & $\begin{array}{l}- \\
\bar{T}\end{array}$ & $\begin{array}{l}- \\
- \\
-\end{array}$ \\
\hline 29 & 319-328 & 321.2 & 73.3 & 58.3 & 85.9 & 2.7 & - & - & & - & - & - & 6.0 & 5.4 & - & & - & - & $\mathrm{T}$ & - \\
\hline \multicolumn{21}{|c|}{ 2-20 $\mu$ Fraction } \\
\hline 2 & $9-18$ & $\begin{array}{l}10.1 \\
14.3\end{array}$ & $\begin{array}{l}81.8 \\
83.1\end{array}$ & $\begin{array}{l}71.5 \\
73.6\end{array}$ & & $\begin{array}{r}14.4 \\
9.5\end{array}$ & - & $\begin{array}{l}51.4 \\
47.8\end{array}$ & & $\begin{array}{l}3.4 \\
3.9\end{array}$ & $\begin{array}{l}1.1 \\
-\end{array}$ & $\begin{array}{r}6.4 \\
14.6\end{array}$ & $\begin{array}{l}- \\
-\end{array}$ & $\begin{array}{r}3.9 \\
14.9\end{array}$ & $\begin{array}{l}0.5 \\
0.7\end{array}$ & & $\begin{array}{r}14.0 \\
4.3\end{array}$ & $\begin{array}{l}4.8 \\
4.3\end{array}$ & - & \\
\hline 3 & $18-27$ & 27.0 & 79.5 & 67.9 & & 2.8 & - & 29.4 & & - & - & 17.6 & - & 46.4 & - & & - & $3: 8$ & - & \\
\hline 5 & $38-47$ & 42.1 & 97.2 & 95.6 & & 5.3 & 26.4 & 50.9 & & - & - & - & - & - & - & & 8.6 & 8.8 & - & \\
\hline 7 & $56-65$ & 56.5 & 91.0 & 85.9 & & 3.6 & - & 62.2 & & - & - & - & - & - & - & & 27.0 & 7.1 & - & \\
\hline 8 & $65-74$ & $\begin{array}{l}67.4 \\
69.0 \\
74.0 \\
\end{array}$ & $\begin{array}{l}90.9 \\
74.9 \\
87.5 \\
\end{array}$ & $\begin{array}{l}85.8 \\
60.8 \\
80.4 \\
\end{array}$ & & $\begin{array}{r}11.7 \\
5.1 \\
5.6 \\
\end{array}$ & $\begin{array}{l}- \\
- \\
-\end{array}$ & $\begin{array}{l}60.8 \\
38.5 \\
69.4\end{array}$ & & $\begin{array}{l}- \\
1.9 \\
-\end{array}$ & $\begin{array}{l}- \\
- \\
-\end{array}$ & $\begin{array}{c}- \\
44.3 \\
-\end{array}$ & $\begin{array}{l}- \\
5.9 \\
-\end{array}$ & $\begin{array}{l}- \\
- \\
-\end{array}$ & $\begin{array}{l}- \\
- \\
-\end{array}$ & & $\begin{array}{c}21.3 \\
- \\
19.1\end{array}$ & $\begin{array}{l}6.3 \\
4.4 \\
5.9\end{array}$ & $\begin{array}{l}- \\
- \\
-\end{array}$ & \\
\hline
\end{tabular}




\begin{tabular}{|c|c|c|c|c|c|c|c|c|c|c|c|c|c|c|c|c|c|c|c|c|c|}
\hline Core & $\begin{array}{c}\text { Cored } \\
\text { Interval } \\
\text { Below Sea } \\
\text { Floor (m) }\end{array}$ & $\begin{array}{l}\text { Sample Deptha } \\
\text { Below Sea } \\
\text { Floor } \\
\text { (m) }\end{array}$ & 㟃 & $\begin{array}{l}\dot{0} \\
\text { 它 } \\
\text { छㄴ }\end{array}$ & $\frac{\dot{v}}{\pi}$ & 㟧 & 这 & 离 & $\begin{array}{l}\overrightarrow{0} \\
\stackrel{0}{\sharp}\end{array}$ & 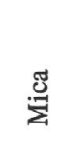 & $\frac{\dot{0}}{\frac{1}{U}}$ & 莣 & 苞 & हं & 要 & 葛 & 家 & 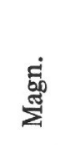 & 这 & $\overrightarrow{5}$ & i \\
\hline 11 & $95-104$ & 97.6 & 90.1 & 84.5 & & 6.3 & - & 47.7 & & 2.2 & - & 12.1 & 1.2 & 6.3 & - & & 19.9 & 4.3 & - & & \\
\hline 12 & $104-113$ & 113.0 & 93.1 & 89.2 & & 6.5 & - & 56.1 & & - & - & 9.4 & - & - & 1.9 & & 21.0 & 5.0 & - & & \\
\hline 15 & $131-140$ & $\begin{array}{l}133.6 \\
134.8 \\
140.0\end{array}$ & $\begin{array}{l}91.5 \\
99.4 \\
88.8\end{array}$ & $\begin{array}{l}86.7 \\
99.1 \\
82.5\end{array}$ & & $\begin{array}{r}10.3 \\
12.3 \\
4.7\end{array}$ & $\begin{array}{l}- \\
- \\
-\end{array}$ & $\begin{array}{l}52.6 \\
35.5 \\
51.7\end{array}$ & & $\overline{9} .6$ & $\begin{array}{l}- \\
- \\
-\end{array}$ & $\begin{array}{c}13.0 \\
- \\
17.8\end{array}$ & $\begin{array}{l}- \\
- \\
1.5\end{array}$ & $\begin{array}{l}- \\
- \\
-\end{array}$ & $\begin{array}{l}1.0 \\
4.8 \\
1.9\end{array}$ & & $\begin{array}{l}18.4 \\
35.6 \\
18.8\end{array}$ & $\begin{array}{l}4.8 \\
2.3 \\
3.5\end{array}$ & $\begin{array}{l}- \\
- \\
-\end{array}$ & & \\
\hline 16 & $142-151$ & 145.8 & 93.1 & 89.2 & & 7.1 & - & 68.3 & & - & - & - & - & - & - & & 20.3 & 4.4 & - & & \\
\hline 17 & $151-160$ & 155.0 & 92.9 & 88.9 & & 10.5 & - & 48.5 & & - & - & 16.8 & 1.5 & - & 2.6 & & 18.1 & 2.0 & - & & \\
\hline 23 & $246-255$ & 255.0 & 80.0 & 68.8 & & 3.3 & - & 47.8 & & - & - & 44.3 & - & - & - & & 0.7 & 3.9 & - & & \\
\hline 24 & $274-283$ & 283.0 & 89.6 & 83.7 & & 10.2 & - & 28.0 & & - & - & 50.8 & - & - & - & & 9.1 & 1.9 & - & & \\
\hline 25 & 283-292 & 292.0 & 77.4 & 64.6 & & 5.0 & - & 58.9 & & - & - & 32.6 & - & - & - & & - & 3.5 & - & & \\
\hline 26 & $292-301$ & $\begin{array}{l}295.6 \\
301.0\end{array}$ & $\begin{array}{l}77.0 \\
81.4\end{array}$ & $\begin{array}{l}64.0 \\
70.9\end{array}$ & & $\begin{array}{r}10.4 \\
7.8\end{array}$ & - & $\begin{array}{l}38.8 \\
17.3\end{array}$ & & $\begin{array}{l}2.4 \\
-\end{array}$ & $\begin{array}{l}- \\
-\end{array}$ & $\begin{array}{l}45.7 \\
72.5\end{array}$ & $\begin{array}{l}2.1 \\
-\end{array}$ & $\begin{array}{l}- \\
-\end{array}$ & - & & $\begin{array}{l}- \\
-\end{array}$ & $\begin{array}{l}0.6 \\
2.4\end{array}$ & - & & \\
\hline 27 & $301-310$ & 310.0 & 89.8 & 84.0 & & 14.3 & - & 22.9 & & 14.7 & - & 34.8 & 2.4 & 11.0 & - & & - & - & - & & \\
\hline 28 & $310-319$ & $\begin{array}{l}311.0 \\
311.4 \\
319.0\end{array}$ & $\begin{array}{l}79.8 \\
81.1 \\
75.6\end{array}$ & $\begin{array}{l}68.4 \\
70.4 \\
61.8\end{array}$ & & $\begin{array}{r}5.4 \\
2.5 \\
13.6\end{array}$ & $\begin{array}{l}- \\
- \\
-\end{array}$ & $\begin{array}{r}32.2 \\
8.9 \\
19.8\end{array}$ & & $\begin{array}{l}9.7 \\
- \\
-\end{array}$ & $\begin{array}{l}- \\
- \\
-\end{array}$ & $\begin{array}{c}49.6 \\
88.5 \\
-\end{array}$ & $\begin{array}{c}\overline{-} \\
\overline{25.2}\end{array}$ & $\begin{array}{c}\overline{-} \\
\overline{41.4}\end{array}$ & $\begin{array}{l}- \\
- \\
-\end{array}$ & & $\begin{array}{l}- \\
- \\
-\end{array}$ & $\begin{array}{l}3.0 \\
- \\
-\end{array}$ & $\begin{array}{l}- \\
\bar{T}\end{array}$ & & \\
\hline 29 & $319-328$ & $\begin{array}{l}321.2 \\
323.0\end{array}$ & $\begin{array}{l}82.8 \\
79.5 \\
\end{array}$ & $\begin{array}{l}73.1 \\
67.9 \\
\end{array}$ & & $\begin{array}{r}12.1 \\
1.2 \\
\end{array}$ & $\begin{array}{l}- \\
- \\
\end{array}$ & $\begin{array}{l}13.2 \\
10.1 \\
\end{array}$ & & - & $\begin{array}{l}- \\
- \\
\end{array}$ & $8 \overline{1.3}$ & $\begin{array}{c}27.5 \\
- \\
\end{array}$ & $\begin{array}{r}47.2 \\
7.3 \\
\end{array}$ & - & & $\begin{array}{l}- \\
- \\
\end{array}$ & - & $\begin{array}{l}\mathrm{T} \\
-\end{array}$ & & \\
\hline \multicolumn{22}{|c|}{$<2 \mu$ Fraction } \\
\hline 2 & $9-18$ & $\begin{array}{l}10.1 \\
14.3\end{array}$ & $\begin{array}{l}85.6 \\
85.5\end{array}$ & $\begin{array}{l}77.6 \\
77.3\end{array}$ & & $\begin{array}{l}8.6 \\
5.3\end{array}$ & $\begin{array}{l}- \\
-\end{array}$ & $\begin{array}{l}27.3 \\
15.3\end{array}$ & $\begin{array}{l}- \\
-\end{array}$ & $\overline{5.5}$ & & $\begin{array}{l}51.2 \\
47.7\end{array}$ & $\begin{array}{l}- \\
-\end{array}$ & $\overline{26.3}$ & - & $\begin{array}{l}- \\
-\end{array}$ & $\begin{array}{c}12.2 \\
-\end{array}$ & $\begin{array}{l}0.7 \\
-\end{array}$ & - & & - \\
\hline 3 & $18-27$ & 27.0 & 81.9 & 71.7 & & 1.6 & - & 11.8 & - & - & & 54.4 & - & 29.2 & - & - & - & 3.0 & - & & - \\
\hline 5 & $38-47$ & 42.1 & 94.7 & 91.7 & & 1.6 & 15.3 & 23.7 & - & - & & 45.8 & - & - & - & - & 7.2 & 6.5 & - & & - \\
\hline 7 & $56-65$ & 56.5 & 97.4 & 96.0 & & 3.8 & - & 42.6 & - & - & & 45.7 & - & - & - & - & - & 8.0 & - & & - \\
\hline 8 & $65-74$ & $\begin{array}{l}67.4 \\
69.0 \\
74.0\end{array}$ & $\begin{array}{l}87.6 \\
97.3 \\
86.0\end{array}$ & $\begin{array}{l}80.6 \\
95.8 \\
78.2\end{array}$ & & $\begin{array}{l}6.0 \\
1.6 \\
1.9\end{array}$ & $\begin{array}{l}- \\
- \\
-\end{array}$ & $\begin{array}{l}36.5 \\
19.4 \\
16.3\end{array}$ & $\begin{array}{l}6.9 \\
- \\
4.7\end{array}$ & $\begin{array}{l}- \\
- \\
-\end{array}$ & & $\begin{array}{l}43.8 \\
27.8 \\
77.1\end{array}$ & $\begin{array}{l}- \\
- \\
-\end{array}$ & $\begin{array}{l}- \\
- \\
-\end{array}$ & $\begin{array}{l}- \\
-\end{array}$ & $\begin{array}{l}- \\
- \\
-\end{array}$ & $\begin{array}{l}- \\
- \\
-\end{array}$ & $\begin{array}{c}6.8 \\
51.2 \\
-\end{array}$ & $\begin{array}{l}- \\
-\end{array}$ & & $\begin{array}{l}- \\
- \\
-\end{array}$ \\
\hline 11 & $95-104$ & 97.6 & 87.5 & 80.5 & & 1.2 & - & 19.4 & - & - & & 61.8 & - & 9.1 & - & - & 7.2 & 1.4 & - & & - \\
\hline 12 & $104-113$ & 113.0 & 88.4 & 81.9 & & 2.1 & - & 14.8 & - & - & & 79.5 & - & - & - & - & - & 3.7 & - & & - \\
\hline 15 & $131-140$ & $\begin{array}{l}133.6 \\
134.8 \\
140.0\end{array}$ & $\begin{array}{l}87.2 \\
86.5 \\
87.4\end{array}$ & $\begin{array}{l}80.1 \\
78.9 \\
80.3\end{array}$ & & $\begin{array}{l}1.6 \\
- \\
1.1\end{array}$ & $\begin{array}{l}- \\
- \\
-\end{array}$ & $\begin{array}{r}14.3 \\
4.4 \\
16.3\end{array}$ & $\begin{array}{l}- \\
- \\
-\end{array}$ & $\begin{array}{l}5.7 \\
- \\
-\end{array}$ & & $\begin{array}{l}75.8 \\
89.0 \\
76.5\end{array}$ & $\begin{array}{l}- \\
- \\
-\end{array}$ & $\begin{array}{l}- \\
- \\
-\end{array}$ & $\begin{array}{l}- \\
\overline{2} .6\end{array}$ & $\begin{array}{l}- \\
5.3 \\
-\end{array}$ & $\begin{array}{l}- \\
- \\
-\end{array}$ & $\begin{array}{l}2.7 \\
1.3 \\
3.4\end{array}$ & $\begin{array}{l}- \\
-\end{array}$ & & $\begin{array}{l}- \\
-\end{array}$ \\
\hline 16 & $142-151$ & 145.8 & 87.8 & 80.9 & & 1.2 & - & 14.9 & - & - & & 72.7 & - & - & 1.6 & - & 8.9 & 0.8 & - & & - \\
\hline 17 & $151-160$ & 155.0 & 86.8 & 79.4 & & 1.5 & - & 8.9 & 4.1 & - & & 78.7 & - & - & - & - & 5.6 & 1.2 & - & & - \\
\hline 23 & $246-255$ & 255.0 & 78.9 & 67.1 & & 2.1 & - & 18.0 & - & - & & 77.0 & - & - & - & - & - & 2.8 & - & & - \\
\hline
\end{tabular}




\begin{tabular}{|c|c|c|c|c|c|c|c|c|c|c|c|c|c|c|c|c|c|c|}
\hline 24 & $274-283$ & $\begin{array}{l}277.7 \\
283.0\end{array}$ & $\begin{array}{l}70.5 \\
80.1\end{array}$ & $\begin{array}{l}54.0 \\
68.9\end{array}$ & $\begin{array}{r}2.7 \\
13.8\end{array}$ & - & $\begin{array}{l}7.0 \\
9.6\end{array}$ & $\begin{array}{l}- \\
-\end{array}$ & $\begin{array}{l}2.4 \\
-\end{array}$ & $\begin{array}{l}82.8 \\
76.6\end{array}$ & $\begin{array}{l}1.2 \\
-\end{array}$ & - & $\begin{array}{l}- \\
-\end{array}$ & $\begin{array}{l}- \\
-\end{array}$ & $\begin{array}{l}3.9 \\
-\end{array}$ & $\begin{array}{l}- \\
-\end{array}$ & $\begin{array}{l}- \\
-\end{array}$ & - \\
\hline 25 & $283-292$ & 292.0 & 71.8 & 55.9 & 1.7 & - & 11.9 & - & - & 85.4 & - & - & - & - & - & 1.0 & - & - \\
\hline 26 & $292-301$ & $\begin{array}{l}295.6 \\
301.0\end{array}$ & $\begin{array}{l}72.2 \\
75.9\end{array}$ & $\begin{array}{l}56.5 \\
62.3\end{array}$ & $\begin{array}{l}4.9 \\
-\end{array}$ & $\begin{array}{l}- \\
-\end{array}$ & $\begin{array}{l}2.3 \\
-\end{array}$ & $\begin{array}{l}- \\
-\end{array}$ & $\begin{array}{l}- \\
-\end{array}$ & $\begin{array}{r}92.8 \\
100.0\end{array}$ & $\begin{array}{l}- \\
-\end{array}$ & $\begin{array}{l}- \\
-\end{array}$ & $\begin{array}{l}- \\
-\end{array}$ & $\begin{array}{l}- \\
-\end{array}$ & $\begin{array}{l}- \\
-\end{array}$ & $\begin{array}{l}- \\
-\end{array}$ & $\begin{array}{l}- \\
-\end{array}$ & $\bar{P}$ \\
\hline 27 & $301-310$ & 310.0 & 85.0 & 76.5 & 6.1 & - & 3.9 & - & 12.3 & 77.7 & - & - & - & - & - & - & - & - \\
\hline 28 & $310-319$ & $\begin{array}{l}311.0 \\
311.4 \\
319.0\end{array}$ & $\begin{array}{l}74.3 \\
68.2 \\
91.9\end{array}$ & $\begin{array}{l}59.9 \\
50.4 \\
87.3\end{array}$ & $\begin{array}{c}- \\
\overline{-} \\
19.4\end{array}$ & $\begin{array}{l}- \\
- \\
-\end{array}$ & $\begin{array}{l}- \\
- \\
-\end{array}$ & $\begin{array}{l}- \\
- \\
-\end{array}$ & $\begin{array}{l}- \\
- \\
-\end{array}$ & $\begin{array}{r}100.0 \\
100.0 \\
67.1\end{array}$ & $\begin{array}{l}- \\
- \\
-\end{array}$ & $\begin{array}{c}- \\
\overline{13.5}\end{array}$ & $\begin{array}{l}- \\
- \\
-\end{array}$ & $\begin{array}{l}- \\
- \\
-\end{array}$ & $\begin{array}{l}- \\
- \\
-\end{array}$ & $\begin{array}{l}- \\
- \\
-\end{array}$ & $\begin{array}{l}- \\
\overline{\mathbf{P}}\end{array}$ & $\begin{array}{l}- \\
- \\
-\end{array}$ \\
\hline 29 & $319-328$ & $\begin{array}{l}321.2 \\
323.0\end{array}$ & $\begin{array}{l}87.7 \\
76.0\end{array}$ & $\begin{array}{l}80.8 \\
62.5\end{array}$ & $\begin{array}{r}15.5 \\
0.7\end{array}$ & $\begin{array}{l}- \\
-\end{array}$ & 10.4 & $\begin{array}{l}- \\
-\end{array}$ & $\begin{array}{l}- \\
-\end{array}$ & $\begin{array}{l}76.6 \\
83.2\end{array}$ & $\begin{array}{l}7.9 \\
-\end{array}$ & $\overline{5.7}$ & $\begin{array}{l}- \\
-\end{array}$ & $\begin{array}{l}- \\
-\end{array}$ & - & $\begin{array}{l}- \\
-\end{array}$ & $\begin{array}{l}\mathbf{P} \\
-\end{array}$ & - \\
\hline
\end{tabular}

aPeaks at $12.1 \AA$.

bPeaks at $2.83 \AA$ and $2.00 \AA ; T=$ trace $; \mathrm{P}=$ presence. 
TABLE 8

Results of X-ray Diffraction Analyses from Hole 206

\begin{tabular}{|c|c|c|c|c|c|c|c|c|c|c|c|c|}
\hline Core & $\begin{array}{c}\text { Cored } \\
\text { Interval } \\
\text { Below Sea } \\
\text { Floor (m) }\end{array}$ & $\begin{array}{l}\text { Sample Deptha } \\
\text { Below Sea } \\
\text { Floor } \\
\text { (m) }\end{array}$ & 岕 & 苞 & $\frac{\tilde{e}}{\tilde{J}}$ & 苟 & 券 & $\begin{array}{l}\overrightarrow{0} \\
\ddot{\Xi}\end{array}$ & 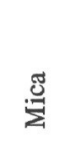 & 总 & $\frac{\dot{\Xi}}{\sum}$ & 客 \\
\hline \multicolumn{13}{|c|}{ Bulk Samples } \\
\hline 1 & $0-4$ & 2.1 & 53.3 & 27.0 & 98.5 & 1.5 & - & & - & & - & \\
\hline 10 & $80-89$ & 82.3 & 53.8 & 27.8 & 91.1 & 3.2 & 1.7 & & 4.1 & & - & \\
\hline 14 & $116-125$ & 118.6 & 49.8 & 21.6 & 98.7 & 1.3 & - & & - & & - & \\
\hline 15 & $125-134$ & 129.1 & 47.5 & 18.0 & 99.3 & 0.7 & - & & - & & - & \\
\hline 18 & $152-161$ & 157.6 & 46.5 & 16.4 & 99.7 & 0.3 & - & & - & & - & \\
\hline 20 & $174-183$ & 176.1 & 45.1 & 16.4 & 100.0 & - & - & & - & & - & \\
\hline 22 & $192-201$ & 196.0 & 47.2 & 17.5 & 99.7 & 0.3 & - & & - & & - & \\
\hline 26 & $228-237$ & 231.6 & 48.7 & 19.8 & 99.7 & 0.3 & - & & - & & - & \\
\hline 31 & $277-286$ & 280.6 & 47.6 & 18.2 & 100.0 & - & - & & - & & - & \\
\hline 34 & $304-313$ & 305.8 & 52.2 & 25.3 & 98.3 & 1.1 & 0.6 & & - & & - & \\
\hline 40 & $362-371$ & 364.1 & 53.5 & 27.4 & 99.1 & 0.9 & - & & - & & - & \\
\hline 44 & $398-407$ & 399.3 & 56.7 & 32.3 & 86.9 & 8.1 & 1.8 & & 1.9 & & 1.2 & \\
\hline \multicolumn{13}{|c|}{ 2-20 $\mu$ Fraction } \\
\hline 10 & $80-89$ & 82.3 & 67.2 & 48.7 & & 38.9 & 26.1 & - & 30.6 & 4.5 & - & - \\
\hline 18 & $152-161$ & 157.6 & 92.6 & 88.4 & & 54.2 & 14.6 & 8.6 & 19.4 & 3.2 & - & - \\
\hline 34 & $304-313$ & 305.8 & 89.4 & 83.4 & & 56.2 & 33.6 & - & 7.6 & 2.6 & - & - \\
\hline 40 & $362-371$ & 364.1 & 89.9 & 84.2 & & 53.0 & 37.9 & - & 5.2 & 2.3 & - & 1.7 \\
\hline 44 & $398-407$ & 399.3 & 70.4 & 53.8 & & 62.7 & 16.6 & - & 10.0 & 3.1 & 7.6 & - \\
\hline \multicolumn{13}{|c|}{$<2 \mu$ Fraction } \\
\hline 1 & $0-4$ & 2.1 & 87.7 & 80.8 & & 31.0 & 8.7 & 25.7 & 34.6 & - & - & \\
\hline 10 & $80-89$ & 82.3 & 91.8 & 87.2 & & 22.3 & 10.7 & 9.8 & 36.9 & 3.8 & 16.5 & \\
\hline 14 & $116-125$ & 118.6 & 86.1 & 78.3 & & 20.7 & 7.0 & 17.3 & 14.2 & 1.6 & 39.2 & \\
\hline 15 & $125-134$ & 129.1 & 86.0 & 78.1 & & 21.2 & 7.4 & 19.2 & 21.3 & 1.9 & 29.1 & \\
\hline 18 & $152-161$ & 157.6 & 90.1 & 84.6 & & 19.6 & 5.5 & 16.7 & 20.3 & 1.4 & 36.6 & \\
\hline 20 & $174-183$ & 176.1 & 90.7 & 85.4 & & 30.2 & 12.3 & 22.6 & 34.9 & - & - & \\
\hline 22 & $192-201$ & 196.0 & 89.4 & 83.4 & & 12.8 & 5.5 & 10.6 & 14.9 & - & 56.1 & \\
\hline 26 & $228-237$ & 231.6 & 83.3 & 74.0 & & 13.5 & 3.4 & 14.2 & 11.2 & 1.7 & 56.0 & \\
\hline 31 & $277-286$ & 280.6 & 87.1 & 79.8 & & 20.2 & 7.0 & 16.5 & 11.4 & 1.7 & 43.3 & \\
\hline 34 & $304-313$ & 305.8 & 81.8 & 71.5 & & 27.2 & 5.6 & 5.6 & 7.2 & 2.5 & 51.9 & \\
\hline 40 & $362-371$ & 364.1 & 88.9 & 82.6 & & 27.4 & 10.8 & 7.7 & 3.4 & - & 50.7 & \\
\hline 44 & $398-407$ & 399.3 & 76.6 & 63.5 & & 59.4 & 6.9 & 2.4 & 3.5 & 1.6 & 26.2 & \\
\hline
\end{tabular}


TABLE 9

Results of X-ray Diffraction Analyses from Hole 206C

\begin{tabular}{|c|c|c|c|c|c|c|c|c|c|c|c|c|c|c|c|c|c|c|}
\hline Core & $\begin{array}{c}\text { Cored } \\
\text { Interval } \\
\text { Below Sea } \\
\text { Floor (m) }\end{array}$ & $\begin{array}{l}\text { Sample Deptha } \\
\text { Below Sea } \\
\text { Floor } \\
\text { (m) }\end{array}$ & 出 & $\begin{array}{l}\dot{2} \\
\dot{0} \\
\dot{E}\end{array}$ & गु & 产 & $\dot{\ddot{g}}$ & 选 & $\stackrel{\varpi 0}{a}$ & 远 & $\stackrel{\Xi}{\Sigma}$ & 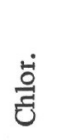 & 莣 & 总 & : & 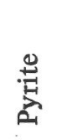 & $\dot{\tilde{\Xi}}$ & 苞 \\
\hline \multicolumn{19}{|c|}{ Bulk Samples } \\
\hline 2 & $413-422$ & 415.0 & 61.3 & 39.5 & 76.1 & 11.7 & - & - & 3.7 & & 5.0 & 1.3 & 2.1 & - & - & & - & \\
\hline 3 & $422-431$ & 424.1 & 60.6 & 38.4 & 76.3 & 11.1 & - & - & 4.3 & & 4.1 & 1.4 & 2.8 & - & - & & - & \\
\hline 4 & $431-440$ & 433.2 & 59.5 & 36.8 & 81.8 & 7.7 & - & - & 2.7 & & 4.3 & 1.2 & 2.4 & - & - & & - & \\
\hline 5 & $440-449$ & 442.3 & 56.2 & 31.5 & 85.9 & 7.7 & - & - & 2.2 & & 2.3 & 0.7 & 1.3 & - & - & & - & \\
\hline 6 & $462-471$ & 466.0 & 57.1 & 33.0 & 85.9 & 6.5 & - & - & 2.7 & & 2.9 & 0.8 & 1.2 & - & - & & - & \\
\hline 7 & $480-489$ & 481.1 & 57.2 & 33.1 & 92.3 & 3.9 & - & - & 1.5 & & 1.1 & - & 1.2 & - & - & & - & \\
\hline 8 & $499-508$ & 499.6 & 57.9 & 34.3 & 85.1 & 6.1 & - & - & 3.2 & & 2.5 & 0.7 & 2.5 & - & - & & - & \\
\hline 10 & $531-540$ & 533.8 & 56.0 & 31.2 & 93.2 & 2.4 & - & - & 1.2 & & 1.4 & - & 1.9 & - & - & & - & \\
\hline 11 & $547-556$ & 554.0 & 57.7 & 33.9 & 88.2 & 3.2 & - & - & - & & 1.4 & - & 7.2 & - & - & & - & \\
\hline 12 & $565-574$ & 567.7 & 56.0 & 31.2 & 92.0 & 2.8 & - & - & - & & - & - & 5.1 & - & - & & - & \\
\hline 13 & $584-593$ & $\begin{array}{l}586.4 \\
587.9\end{array}$ & $\begin{array}{l}54.5 \\
54.5\end{array}$ & $\begin{array}{l}28.9 \\
28.9\end{array}$ & $\begin{array}{l}95.5 \\
97.5\end{array}$ & $\begin{array}{l}1.9 \\
1.4\end{array}$ & $\begin{array}{l}- \\
-\end{array}$ & - & - & & $\begin{array}{l}- \\
-\end{array}$ & - & $\begin{array}{l}2.6 \\
1.1\end{array}$ & $\begin{array}{l}- \\
-\end{array}$ & $\begin{array}{l}- \\
-\end{array}$ & & - & \\
\hline 14 & $603-612$ & 605.4 & 55.0 & 29.7 & 94.3 & 1.9 & - & - & - & & - & - & 2.7 & - & 1.1 & & - & \\
\hline 15 & $612-621$ & 614.1 & 63.3 & 42.6 & 90.9 & 1.9 & - & - & - & & - & - & 7.2 & - & - & & - & \\
\hline 16 & $630-639$ & $\begin{array}{l}631.4 \\
635.1\end{array}$ & $\begin{array}{l}56.9 \\
52.1\end{array}$ & $\begin{array}{l}32.6 \\
25.2\end{array}$ & $\begin{array}{l}94.3 \\
98.0\end{array}$ & $\begin{array}{l}1.0 \\
0.5\end{array}$ & - & - & - & & - & - & $\begin{array}{l}4.7 \\
1.5\end{array}$ & $\begin{array}{l}- \\
-\end{array}$ & - & & - & \\
\hline 17 & $649-658$ & $\begin{array}{l}652.7 \\
657.7\end{array}$ & $\begin{array}{l}69.1 \\
66.5\end{array}$ & $\begin{array}{l}51.7 \\
47.7\end{array}$ & $\begin{array}{l}93.0 \\
97.9\end{array}$ & $\begin{array}{l}1.1 \\
0.7\end{array}$ & $\begin{array}{l}- \\
-\end{array}$ & - & - & & $\begin{array}{l}- \\
-\end{array}$ & - & $\begin{array}{l}5.9 \\
1.3\end{array}$ & $\begin{array}{l}- \\
-\end{array}$ & - & & - & \\
\hline 18 & $668-677$ & 670.7 & 68.7 & 51.0 & 95.4 & 1.1 & - & - & - & & - & - & 3.5 & - & - & & - & \\
\hline 19 & $687-696$ & 688.3 & 51.1 & 23.6 & 98.3 & - & - & - & - & & - & - & 1.7 & - & - & & - & \\
\hline 20 & 706-715 & 706.9 & 62.7 & 41.6 & 86.9 & 2.4 & - & - & - & & 2.0 & - & 2.3 & - & 6.3 & & - & \\
\hline 21 & $725-734$ & $\begin{array}{l}725.7 \\
727.3 \\
\end{array}$ & $\begin{array}{l}83.6 \\
59.9 \\
\end{array}$ & $\begin{array}{l}74.4 \\
37.3 \\
\end{array}$ & $\begin{array}{l}22.0 \\
84.3 \\
\end{array}$ & $\begin{array}{l}3.7 \\
1.0 \\
\end{array}$ & $\begin{array}{r}52.4 \\
9.9 \\
\end{array}$ & $\begin{array}{c}0.6 \\
- \\
\end{array}$ & $\begin{array}{c}1.3 \\
- \\
\end{array}$ & & $\begin{array}{c}2.9 \\
- \\
\end{array}$ & $\begin{array}{l}- \\
-\end{array}$ & $\begin{array}{l}8.2 \\
1.1 \\
\end{array}$ & $\begin{array}{l}7.6 \\
2.5 \\
\end{array}$ & $\overline{1.3}$ & & $\begin{array}{c}1.4 \\
- \\
\end{array}$ & \\
\hline \multicolumn{19}{|c|}{ 2-20 $\mu$ Fraction } \\
\hline 2 & $413-422$ & 415.0 & 71.6 & 55.6 & & 49.5 & - & - & 24.6 & - & 16.8 & 8.1 & - & - & - & - & - & 1.1 \\
\hline 3 & $422-431$ & 424.1 & 67.5 & .49 .3 & & 53.0 & - & - & 21.6 & - & 14.1 & 4.7 & 6.7 & - & - & - & - & - \\
\hline 4 & $431-440$ & 433.2 & 69.3 & 52.0 & & 48.1 & - & - & 29.9 & - & 16.9 & 5.1 & - & - & - & - & - & - \\
\hline 5 & $440-449$ & 442.3 & 73.3 & 58.3 & & 46.7 & - & - & 20.7 & - & 15.5 & 4.4 & 12.7 & - & - & - & - & - \\
\hline 6 & $462-471$ & 466.0 & 73.8 & 59.1 & & 39.1 & - & - & 24.0 & - & 16.1 & 6.6 & 14.2 & - & - & - & - & - \\
\hline 7 & $480-489$ & 481.1 & 77.3 & 64.5 & & 43.6 & - & - & 19.1 & 0.9 & 10.1 & 3.7 & 22.6 & - & - & - & - & - \\
\hline 8 & 499-508 & 499.6 & 74.2 & 59.7 & & 45.4 & - & - & 28.2 & - & 12.4 & 5.2 & 8.7 & - & - & - & - & - \\
\hline 10 & $531-540$ & 533.8 & 77.9 & 65.5 & & 43.2 & - & 5.1 & 16.7 & - & 6.5 & 1.3 & 26.3 & - & 1.0 & - & - & - \\
\hline 11 & $547-556$ & 554.0 & 78.2 & 65.9 & & 42.9 & - & 3.1 & 14.4 & - & 5.3 & 1.8 & 30.0 & - & - & 2.5 & - & - \\
\hline 12 & $565-574$ & 567.7 & 88.2 & 81.6 & & 67.0 & - & - & 24.5 & - & 4.6 & 0.8 & - & - & 3.1 & - & - & - \\
\hline 13 & $584-593$ & $\begin{array}{l}586.4 \\
587.9\end{array}$ & $\begin{array}{l}74.8 \\
72.4\end{array}$ & $\begin{array}{l}60.6 \\
56.8\end{array}$ & & $\begin{array}{l}50.0 \\
47.6\end{array}$ & - & $\begin{array}{l}3.4 \\
5.1\end{array}$ & $\begin{array}{l}14.3 \\
14.2\end{array}$ & - & $\begin{array}{l}6.3 \\
8.7\end{array}$ & $\begin{array}{l}7.8 \\
2.4\end{array}$ & - & $\begin{array}{l}- \\
-\end{array}$ & $\begin{array}{l}18.1 \\
20.6\end{array}$ & $\begin{array}{l}- \\
-\end{array}$ & - & - \\
\hline 14 & $603-612$ & 605.4 & 71.9 & 56.1 & & 46.8 & - & 3.9 & 13.9 & - & 4.8 & 1.1 & - & - & 29.4 & - & - & - \\
\hline 15 & $61.2-621$ & 614.1 & 90.7 & 85.4 & & 20.5 & - & 2.8 & 21.4 & - & 3.9 & 0.8 & 42.0 & - & 2.3 & - & 6.3 & - \\
\hline 16 & $630-639$ & $\begin{array}{l}631.4 \\
635.1\end{array}$ & $\begin{array}{l}89.6 \\
89.4\end{array}$ & $\begin{array}{l}83.8 \\
83.4\end{array}$ & & $\begin{array}{l}23.3 \\
18.4\end{array}$ & $\begin{array}{l}- \\
-\end{array}$ & $\begin{array}{l}5.4 \\
3.8\end{array}$ & $\begin{array}{l}16.7 \\
11.2\end{array}$ & - & $\begin{array}{l}3.5 \\
5.9\end{array}$ & $\begin{array}{l}1.1 \\
0.9\end{array}$ & $\begin{array}{l}48.8 \\
54.0\end{array}$ & $\begin{array}{l}- \\
-\end{array}$ & $\begin{array}{l}1.1 \\
1.2\end{array}$ & $\begin{array}{l}- \\
-\end{array}$ & $-\overline{4.6}$ & - \\
\hline 17 & $649-658$ & $\begin{array}{l}652.7 \\
657.7\end{array}$ & $\begin{array}{l}93.7 \\
95.4\end{array}$ & $\begin{array}{l}90.2 \\
92.8\end{array}$ & & $\begin{array}{l}16.8 \\
22.8\end{array}$ & $\begin{array}{l}- \\
-\end{array}$ & $\begin{array}{c}3.6 \\
-\end{array}$ & $\begin{array}{r}9.1 \\
12.2\end{array}$ & - & $\begin{array}{c}2.2 \\
-\end{array}$ & $\begin{array}{l}1.4 \\
1.7\end{array}$ & $\begin{array}{l}59.9 \\
57.6\end{array}$ & $\begin{array}{l}- \\
-\end{array}$ & - & - & $\begin{array}{l}7.0 \\
5.7\end{array}$ & - \\
\hline 18 & $668-677$ & 670.7 & 95.7 & 93.2 & & 25.3 & - & - & 15.7 & - & 3.2 & - & 49.1 & - & 1.1 & - & 5.5 & - \\
\hline 19 & $687-696$ & 688.3 & 89.4 & 83.4 & & 5.6 & - & - & - & - & - & - & - & - & 44.0 & - & 50.4 & - \\
\hline 21 & $725-734$ & $\begin{array}{l}725.7 \\
727.3\end{array}$ & $\begin{array}{l}83.9 \\
78.2\end{array}$ & $\begin{array}{l}74.8 \\
66.0\end{array}$ & & $\begin{array}{l}9.5 \\
6.4\end{array}$ & $\begin{array}{l}65.1 \\
57.1\end{array}$ & $\begin{array}{l}4.1 \\
1.1\end{array}$ & $\begin{array}{l}4.3 \\
2.0\end{array}$ & $\begin{array}{l}- \\
-\end{array}$ & $\begin{array}{l}4.5 \\
2.6\end{array}$ & $\begin{array}{l}- \\
-\end{array}$ & $\begin{array}{l}- \\
-\end{array}$ & $\begin{array}{r}9.7 \\
10.2\end{array}$ & 17.5 & $\begin{array}{l}- \\
-\end{array}$ & $\begin{array}{l}2.8 \\
3.1\end{array}$ & - \\
\hline
\end{tabular}




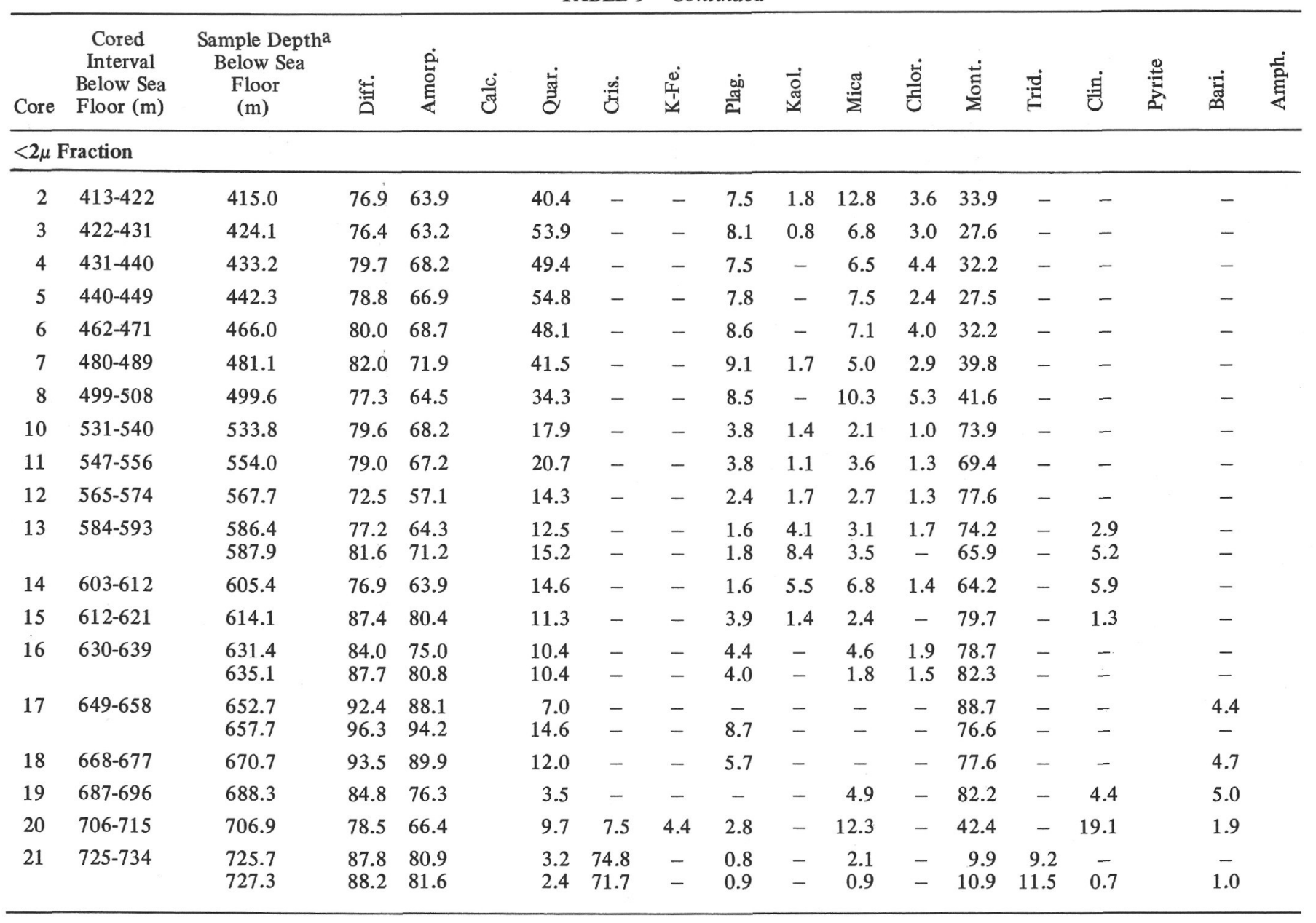

TABLE 10

Results of X-ray Diffraction Analyses from Hole 207

\begin{tabular}{|c|c|c|c|c|c|c|c|c|c|c|c|}
\hline Core & $\begin{array}{l}\text { Cored } \\
\text { Interval } \\
\text { Below Sea } \\
\text { Floor (m) }\end{array}$ & $\begin{array}{l}\text { Sample Deptha } \\
\text { Below Sea } \\
\text { Floor } \\
\text { (m) }\end{array}$ & 出 & 官 & $\frac{\dot{\Xi}}{\tilde{J}}$ & 苟 & $\frac{00}{2}$ & 苛 & 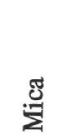 & 莽 & 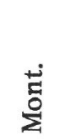 \\
\hline \multicolumn{12}{|c|}{ Bulk Samples } \\
\hline 3 & $15-24$ & 19.5 & 53.5 & 27.4 & 98.9 & 1.1 & & & & & \\
\hline \multicolumn{12}{|c|}{$<2 \mu$ Fraction } \\
\hline 3 & $15-24$ & 19.5 & 89.5 & 83.5 & & 24.7 & 11.7 & 9.8 & 37.1 & 6.0 & 10.7 \\
\hline
\end{tabular}


TABLE 11

Results of X-ray Diffraction Analyses from Hole 207A

\begin{tabular}{|c|c|c|c|c|c|c|c|c|c|c|c|c|c|}
\hline Core & $\begin{array}{c}\text { Cored } \\
\text { Interval } \\
\text { Below Sea } \\
\text { Floor (m) }\end{array}$ & $\begin{array}{l}\text { Sample Deptha } \\
\text { Below Sea } \\
\text { Floor } \\
\text { (m) }\end{array}$ & 出 & 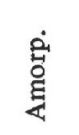 & $\ddot{\Xi}$ & 总 & 选 & $\frac{\dot{m}}{2}$ & $\stackrel{\overparen{Z}}{\check{\Sigma}}$ & $\begin{array}{l}+ \\
\frac{0}{0}\end{array}$ & 莡 & 竎 & 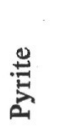 \\
\hline \multicolumn{14}{|c|}{ Bulk Samples } \\
\hline 33 & $348-357$ & $\begin{array}{l}349.5 \\
351.1\end{array}$ & $\begin{array}{l}86.4 \\
87.2\end{array}$ & $\begin{array}{l}78.8 \\
80.1\end{array}$ & $\begin{array}{l}8.4 \\
5.9\end{array}$ & $\begin{array}{l}69.2 \\
69.2\end{array}$ & $\begin{array}{l}1.5 \\
1.4\end{array}$ & $\begin{array}{l}3.8 \\
2.3\end{array}$ & $\begin{array}{l}1.6 \\
0.9\end{array}$ & $\begin{array}{l}3.7 \\
3.0\end{array}$ & $\begin{array}{r}8.5 \\
14.4\end{array}$ & $\begin{array}{l}1.4 \\
0.9\end{array}$ & $\begin{array}{l}1.8 \\
1.9\end{array}$ \\
\hline 42 & $432-441$ & 434.5 & 73.0 & 57.9 & 0.6 & 5.5 & - & 4.6 & - & 89.3 & - & - & - \\
\hline 46 & $468-477$ & 471.7 & 82.9 & 73.2 & 8.1 & 38.7 & - & 20.3 & - & 32.9 & - & - & - \\
\hline \multicolumn{14}{|c|}{$2-20 \mu$ Fraction } \\
\hline 33 & $348-357$ & $\begin{array}{l}349.5 \\
351.1\end{array}$ & $\begin{array}{l}81.1 \\
81.2\end{array}$ & $\begin{array}{l}70.5 \\
70.6\end{array}$ & $\begin{array}{l}14.9 \\
21.6\end{array}$ & $\begin{array}{l}60.5 \\
42.6\end{array}$ & $\begin{array}{l}2.8 \\
5.1\end{array}$ & $\begin{array}{r}6.8 \\
10.6\end{array}$ & $\begin{array}{l}3.0 \\
2.1\end{array}$ & $\begin{array}{l}- \\
-\end{array}$ & $\begin{array}{l}7.4 \\
6.5\end{array}$ & $\begin{array}{l}2.3 \\
6.0\end{array}$ & $\begin{array}{l}2.3 \\
5.5\end{array}$ \\
\hline 42 & $432-441$ & 434.5 & 80.2 & 69.1 & 1.2 & 23.9 & - & 21.8 & - & 51.3 & 1.9 & - & - \\
\hline 46 & $468-477$ & 471.7 & 88.2 & 81.6 & 3.1 & 38.3 & - & 10.3 & - & 46.4 & 1.3 & 0.6 & - \\
\hline \multicolumn{14}{|c|}{$<2 \mu$ Fraction } \\
\hline 33 & $348-357$ & $\begin{array}{l}349.5 \\
351.1\end{array}$ & $\begin{array}{l}87.9 \\
88.9\end{array}$ & $\begin{array}{l}81.1 \\
82.6\end{array}$ & $\begin{array}{l}0.7 \\
0.9\end{array}$ & $\begin{array}{l}79.0 \\
79.1\end{array}$ & $\overline{1.0}$ & $\begin{array}{l}0.7 \\
0.7\end{array}$ & & $\begin{array}{r}10.0 \\
5.0\end{array}$ & $\begin{array}{r}7.6 \\
11.3\end{array}$ & $\overline{0.5}$ & $\begin{array}{l}1.9 \\
1.5\end{array}$ \\
\hline 42 & $432-441$ & 434.5 & 74.8 & 60.7 & - & 6.4 & - & - & & 93.6 & - & - & - \\
\hline 46 & $468-477$ & 471.7 & 69.3 & 52.1 & - & 4.1 & - & - & & 95.8 & - & - & - \\
\hline
\end{tabular}

TABLE 12

Results of X-ray Diffraction Analyses from Hole 208

\begin{tabular}{|c|c|c|c|c|c|c|c|c|c|c|c|c|c|c|}
\hline Core & $\begin{array}{l}\text { Cored } \\
\text { Interval } \\
\text { Below Sea } \\
\text { Floor (m) }\end{array}$ & $\begin{array}{l}\text { Sample Depth } \\
\text { Below Sea } \\
\text { Floor } \\
\text { (m) }\end{array}$ & 苟 & $\begin{array}{l}\dot{2} \\
\text { है }\end{array}$ & $\frac{\dot{0}}{\tilde{J}}$ & 惌 & 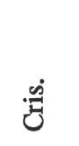 & 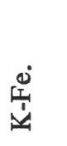 & 离 & 疍 & 范 & 总 & 悤 & 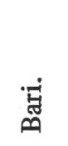 \\
\hline \multicolumn{15}{|c|}{ Bulk Samples } \\
\hline 30 & $548-557$ & 551.9 & 61.2 & 39.4 & 99.6 & 0.4 & - & - & - & - & - & - & - & - \\
\hline 33 & $576-585$ & 579.9 & 54.7 & 29.2 & 88.3 & 0.5 & 7.4 & & & & & 2.6 & 1.2 & \\
\hline 34 & $585-594$ & 589.3 & 52.4 & 25.6 & 91.8 & 0.5 & 6.5 & & & & & - & 1.2 & - \\
\hline \multicolumn{15}{|c|}{ 2-20 $\mu$ Fractions } \\
\hline 30 & $584-557$ & 551.9 & 95.0 & 92.2 & - & 26.8 & - & - & - & - & 39.0 & - & - & 34.2 \\
\hline 33 & $576-585$ & 579.9 & 85.6 & 77.6 & - & 7.9 & 64.4 & 1.9 & 2.0 & 1.5 & - & 6.5 & 14.0 & 1.8 \\
\hline 34 & $585-594$ & 589.3 & 74.2 & 59.7 & - & 17.1 & 11.4 & 2.4 & 3.5 & 2.9 & - & 6.4 & 48.2 & 8.1 \\
\hline \multicolumn{15}{|c|}{$<2 \mu$ Fractions } \\
\hline 30 & $548-557$ & 551.9 & 93.9 & 90.5 & - & 9.3 & - & - & - & - & 76.2 & - & - & 14.5 \\
\hline 33 & $576-585$ & 579.9 & 91.6 & 86.8 & - & 2.4 & 70.2 & 1.5 & - & 1.5 & 10.8 & 11.2 & 0.7 & 1.7 \\
\hline 34 & 585-594 & 589.3 & 88.6 & 82.2 & - & 3.4 & 70.8 & 2.1 & 1.4 & - & 10.3 & 8.3 & 1.3 & 2.6 \\
\hline
\end{tabular}


TABLE 13

Results of X-ray Diffraction Analyses from Hole 209

\begin{tabular}{|c|c|c|c|c|c|c|c|c|c|c|c|c|c|c|c|c|c|c|c|c|c|}
\hline Core & $\begin{array}{l}\text { Cored } \\
\text { Interval } \\
\text { Below Sea } \\
\text { Floor (m) }\end{array}$ & $\begin{array}{l}\text { Sample Depth } \\
\text { Below Sea } \\
\text { Floor } \\
\text { (m) }\end{array}$ & 我 & $\begin{array}{l}\dot{2} \\
\text { 菌 }\end{array}$ & $\frac{\dot{0}}{\pi}$ & 苛 & 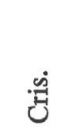 & 䢓 & 富 & $\begin{array}{l}\overrightarrow{0} \\
\ddot{\Xi}\end{array}$ & 苞 & 莺 & 苞 & 萜 & 苞 & 百 & 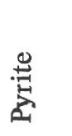 & 帘 & 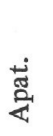 & 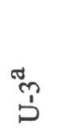 & 'י \\
\hline \multicolumn{22}{|c|}{ Bulk Samples } \\
\hline 1 & $0-9$ & 5.6 & 53.7 & 27.7 & 99.1 & 0.9 & - & - & - & - & - & - & - & - & - & - & - & - & - & - & - \\
\hline 2 & $9-18$ & 14.7 & 51.5 & 24.3 & 99.7 & 0.3 & - & - & - & - & - & - & - & - & - & - & - & - & - & - & - \\
\hline 3 & $18-27$ & 23.7 & 50.4 & 22.5 & 100.0 & - & - & - & - & - & - & - & - & - & - & - & - & - & - & - & - \\
\hline 4 & $27-36$ & 32.7 & 50.7 & 22.9 & 99.6 & 0.4 & - & - & - & - & - & - & - & - & - & - & - & - & - & - & - \\
\hline 5 & $36-45$ & 41.7 & 51.3 & 23.9 & 99.5 & 0.5 & - & - & - & - & - & - & - & - & - & - & - & - & - & - & - \\
\hline 6 & $45-54$ & 52.2 & 52.9 & 26.5 & 99.6 & 0.4 & - & - & - & - & - & - & - & - & - & - & - & - & - & - & - \\
\hline 9 & $74-83$ & 75.3 & 53.6 & 27.4 & 100.0 & - & - & - & - & - & - & - & - & - & - & - & - & - & - & - & - \\
\hline 14 & $131-140$ & 133.7 & 47.6 & 18.1 & 100.0 & - & - & - & - & - & - & - & - & - & - & - & - & - & - & - & - \\
\hline 15 & $150-159$ & 151.0 & 52.8 & 26.3 & 92.7 & 4.3 & 3.1 & - & - & - & - & - & - & - & - & - & - & - & - & - & - \\
\hline 23 & $241-250$ & 243.6 & 68.3 & 50.5 & 98.6 & 1.4 & - & - & - & - & - & - & - & - & - & - & - & - & - & $\mathbf{P}$ & - \\
\hline 27 & $272-281$ & 274.1 & 62.6 & 41.6 & 82.6 & 3.8 & 9.1 & - & - & - & - & - & - & - & 4.5 & - & - & - & - & - & - \\
\hline 28 & $281-290$ & 283.3 & 67.8 & 49.7 & 68.9 & 8.6 & 10.9 & - & 4.6 & - & - & - & - & - & 5.1 & 1.9 & - & - & - & - & - \\
\hline 29 & $290-299$ & 291.3 & 66.1 & 47.0 & 62.6 & 15.2 & 2.8 & 4.2 & 10.1 & - & 2.1 & - & - & 0.9 & 0.6 & 1.5 & - & - & - & - & - \\
\hline 31 & $308-317$ & 309.2 & 77.6 & 64.9 & 35.5 & 16.1 & 24.4 & - & 10.1 & - & 3.8 & - & - & 2.1 & 5.7 & 1.5 & - & 0.7 & - & - & - \\
\hline 32 & $317-326$ & 318.3 & 77.2 & 64.4 & 40.4 & 12.4 & 26.1 & - & 8.6 & - & 4.3 & - & - & 2.4 & 4.7 & - & 1.1 & - & - & - & - \\
\hline 34 & $335-344$ & 337.5 & 73.3 & 58.2 & 91.1 & 2.3 & 2.8 & - & 1.8 & - & 0.7 & - & - & - & 0.8 & 0.5 & - & - & - & - & - \\
\hline \multicolumn{22}{|c|}{ 2-20 $\mu$ Fraction } \\
\hline 23 & $241-250$ & 243.6 & 97.7 & 96.5 & - & 57.8 & - & 17.1 & 14.9 & - & 10.2 & - & - & - & - & - & - & - & - & - & - \\
\hline 27 & $272-281$ & 274.1 & 87.8 & 80.9 & - & 12.4 & 58.8 & - & 4.6 & - & - & 17.8 & - & - & - & 6.3 & - & - & - & - & - \\
\hline 28 & $281-290$ & 283.3 & 84.1 & 75.1 & - & 13.3 & 50.1 & - & 6.7 & - & 3.0 & 13.5 & - & - & - & 12.1 & 1.2 & - & - & - & - \\
\hline 29 & $290-299$ & 291.3 & 73.2 & 58.1 & - & 38.2 & - & 5.8 & 25.4 & - & 8.1 & - & - & - & - & 18.9 & 2.5 & 1.2 & - & - & - \\
\hline 31 & $308-317$ & 309.2 & 81.4 & 70.9 & - & 23.6 & 39.8 & 3.3 & 17.0 & - & 4.3 & 6.6 & - & - & - & 3.8 & 1.7 & - & - & - & - \\
\hline 32 & $317-326$ & 318.3 & 82.2 & 72.2 & - & 22.9 & 41.4 & - & 17.6 & - & 6.4 & 6.5 & - & - & - & 3.7 & 1.6 & - & - & - & - \\
\hline 34 & $335-344$ & 337.5 & 79.3 & 67.7 & - & 22.8 & 33.2 & 4.3 & 17.4 & - & 6.9 & 6.7 & - & - & - & 6.5 & 2.2 & - & - & - & - \\
\hline
\end{tabular}




\begin{tabular}{|c|c|c|c|c|c|c|c|c|c|c|c|c|c|c|c|c|c|c|c|c|c|}
\hline \multicolumn{22}{|c|}{$<2 \mu$ Fraction } \\
\hline 1 & $0-9$ & 5.6 & 85.0 & 76.6 & - & 11.7 & - & - & 8.8 & 12.7 & 13.5 & - & 3.2 & 50.0 & - & - & - & - & - & - & - \\
\hline 2 & $9-18$ & 14.7 & 90.9 & 85.7 & - & 12.8 & - & - & 20.0 & 14.6 & 14.2 & - & - & 38.4 & - & - & - & - & - & - & - \\
\hline 3 & $18-27$ & 23.7 & 97.2 & 95.6 & - & 14.9 & - & - & 12.0 & 16.5 & 19.3 & - & - & 37.4 & - & - & - & - & - & - & - \\
\hline 4 & $27-36$ & 32.7 & 87.9 & 81.0 & - & 11.0 & - & - & 2.8 & 27.6 & 19.9 & - & - & 38.8 & - & - & - & - & - & - & - \\
\hline 5 & $36-45$ & 41.7 & 90.6 & 85.3 & - & 12.4 & - & - & 3.1 & 24.0 & 18.5 & - & - & 41.9 & - & - & - & - & - & - & - \\
\hline 6 & $45-54$ & 52.2 & 98.6 & 97.9 & - & 15.5 & - & - & - & 25.7 & - & - & - & 58.7 & - & - & - & - & - & - & - \\
\hline 14 & $131-140$ & 133.7 & 98.9 & 98.2 & - & 10.9 & - & - & 11.7 & - & - & - & - & 35.1 & - & - & - & - & 42.3 & - & - \\
\hline 15 & $150-159$ & 151.0 & 92.7 & 88.6 & - & 8.3 & 28.4 & - & 1.9 & - & 4.9 & - & - & 33.7 & 6.1 & 1.7 & - & - & 15.0 & - & - \\
\hline 23 & $241-250$ & 243.6 & 91.7 & 87.1 & - & 7.1 & - & - & 3.2 & 9.6 & 8.4 & - & - & 62.3 & - & - & 1.6 & - & 7.8 & - & - \\
\hline 27 & $272-281$ & 274.1 & 90.1 & 84.5 & - & 2.0 & 68.5 & - & - & - & 2.5 & - & - & 11.1 & 15.0 & 0.9 & - & - & - & - & - \\
\hline 28 & $281-290$ & 283.3 & 91.2 & 86.3 & - & 1.5 & 63.3 & - & 1.8 & - & 2.3 & - & - & 9.6 & 14.9 & 1.2 & - & - & 5.5 & - & - \\
\hline 29 & $290-299$ & 291.3 & 86.7 & 792 & - & 5.1 & 21.6 & 2.4 & 7.5 & - & 8.3 & - & - & 23.5 & 17.4 & 5.9 & 2.4 & - & 5.9 & - & $\mathrm{T}$ \\
\hline 31 & $308-317$ & 309.2 & 88.5 & 82.0 & - & 1.7 & 64.2 & - & 0.9 & - & 3.3 & - & - & 11.6 & 16.9 & - & 1.4 & - & - & - & - \\
\hline 32 & $317-326$ & 318.3 & 89.2 & 83.1 & - & 1.5 & 64.3 & - & 0.7 & - & 2.0 & - & - & 15.5 & 13.4 & 1.1 & 1.6 & - & - & - & - \\
\hline 34 & $335-344$ & 337.5 & 87.6 & 80.6 & - & 1.6 & 64.8 & - & 0.9 & - & 2.3 & - & - & 10.5 & 16.2 & 2.2 & 1.6 & - & - & - & - \\
\hline
\end{tabular}

${ }^{\mathrm{a}}$ Peaks at $3.23 \AA$.

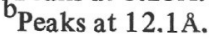


TABLE 14

Results of X-ray Diffraction Analyses from Hole 210

\begin{tabular}{|c|c|c|c|c|c|c|c|c|c|c|c|c|c|c|c|c|c|c|c|c|c|c|}
\hline Core & $\begin{array}{l}\text { Cored } \\
\text { Interval } \\
\text { Below Sea } \\
\text { Floor (m) }\end{array}$ & $\begin{array}{l}\text { Sample Depth } \\
\text { Below Sea } \\
\text { Floor } \\
\text { (m) }\end{array}$ & 出 & 客 & $\frac{0}{\pi}$ & 용 & 妥 & 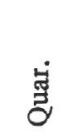 & 离 & 浣 & 蛋 & 官 & 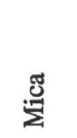 & 运 & 莣 & 总 & 岀 & 咅 & 莺 & 空 & $\frac{\pi}{5}$ & ? \\
\hline \multicolumn{23}{|c|}{ Bulk Samples } \\
\hline 1 & $0-9$ & $\begin{array}{l}0.3 \\
4.7 \\
8.8\end{array}$ & $\begin{array}{l}79.9 \\
80.8 \\
79.9\end{array}$ & $\begin{array}{l}68.7 \\
70.0 \\
68.7\end{array}$ & $\begin{array}{r}61.6 \\
2.3 \\
3.4\end{array}$ & $\begin{array}{l}- \\
- \\
-\end{array}$ & $\begin{array}{l}- \\
- \\
-\end{array}$ & $\begin{array}{l}12.3 \\
31.4 \\
33.3\end{array}$ & $\begin{array}{l}- \\
- \\
-\end{array}$ & $\begin{array}{l}- \\
- \\
-\end{array}$ & $\begin{array}{c}- \\
18.6 \\
24.7\end{array}$ & $\begin{array}{l}2.1 \\
5.4 \\
3.7\end{array}$ & $\begin{array}{l}13.7 \\
34.4 \\
28.1\end{array}$ & $\begin{array}{l}2.9 \\
5.3 \\
4.2\end{array}$ & $\begin{array}{l}7.5 \\
2.6 \\
-\end{array}$ & $\begin{array}{l}- \\
- \\
-\end{array}$ & $\begin{array}{l}- \\
- \\
-\end{array}$ & $\begin{array}{l}- \\
- \\
-\end{array}$ & $\begin{array}{l}- \\
\overline{1.0}\end{array}$ & $\begin{array}{l}- \\
- \\
1.6\end{array}$ & $\begin{array}{l}- \\
- \\
-\end{array}$ & $\begin{array}{l}- \\
- \\
-\end{array}$ \\
\hline 2 & $9-18$ & $\begin{array}{r}9.4 \\
12.8 \\
16.8 \\
17.0\end{array}$ & $\begin{array}{l}74.9 \\
73.3 \\
81.5 \\
83.9\end{array}$ & $\begin{array}{l}60.8 \\
58.3 \\
71.1 \\
74.9\end{array}$ & $\begin{array}{c}- \\
\overline{9.0} \\
11.6\end{array}$ & $\begin{array}{l}- \\
- \\
- \\
-\end{array}$ & $\begin{array}{l}- \\
- \\
- \\
-\end{array}$ & $\begin{array}{l}38.9 \\
43.5 \\
27.4 \\
23.5\end{array}$ & $\begin{array}{l}- \\
- \\
-\end{array}$ & $\begin{array}{l}- \\
\overline{4} \\
- \\
-\end{array}$ & $\begin{array}{l}28.8 \\
36.4 \\
23.9 \\
16.2\end{array}$ & $\begin{array}{l}- \\
\overline{1.2} \\
2.4\end{array}$ & $\begin{array}{l}22.8 \\
12.7 \\
24.0 \\
29.2\end{array}$ & $\begin{array}{l}5.4 \\
3.4 \\
5.9 \\
5.8\end{array}$ & $\begin{array}{l}1.8 \\
1.1 \\
3.1 \\
8.2\end{array}$ & $\begin{array}{l}- \\
- \\
- \\
-\end{array}$ & $\begin{array}{l}- \\
- \\
- \\
-\end{array}$ & $\begin{array}{l}- \\
- \\
- \\
-\end{array}$ & $\begin{array}{l}- \\
- \\
- \\
-\end{array}$ & $\begin{array}{l}2.4 \\
2.9 \\
1.6 \\
3.1\end{array}$ & $\begin{array}{l}- \\
- \\
-\end{array}$ & $\begin{array}{l}- \\
- \\
- \\
-\end{array}$ \\
\hline 3 & $18-27$ & $\begin{array}{l}21.1 \\
23.2\end{array}$ & $\begin{array}{l}79.7 \\
70.1\end{array}$ & $\begin{array}{l}68.3 \\
53.3\end{array}$ & $\begin{array}{r}3.9 \\
32.6\end{array}$ & $\overline{-}$ & $\overline{30.1}$ & $\begin{array}{l}42.2 \\
18.5\end{array}$ & $\begin{array}{l}- \\
-\end{array}$ & $\overline{1.8}$ & $\begin{array}{r}23.4 \\
6.2\end{array}$ & $\begin{array}{l}2.1 \\
0.6\end{array}$ & $\begin{array}{r}18.5 \\
7.7\end{array}$ & $\begin{array}{l}4.8 \\
1.2\end{array}$ & $\begin{array}{l}2.7 \\
1.2\end{array}$ & $\begin{array}{l}- \\
-\end{array}$ & - & - & $\begin{array}{l}1.1 \\
-\end{array}$ & $\begin{array}{l}1.3 \\
-\end{array}$ & - & - \\
\hline 6 & $45-54$ & 49.4 & 77.7 & 65.2 & 58.8 & - & 11.6 & 11.1 & - & - & 2.9 & 3.9 & 9.9 & 1.8 & - & - & - & - & - & - & - & - \\
\hline 8 & $63-72$ & $\begin{array}{l}69.7 \\
70.2\end{array}$ & $\begin{array}{l}81.4 \\
66.0\end{array}$ & $\begin{array}{l}71.0 \\
46.9\end{array}$ & $\begin{array}{r}3.6 \\
26.4\end{array}$ & $\begin{array}{l}- \\
-\end{array}$ & $\overline{54.3}$ & $\begin{array}{l}29.1 \\
12.1\end{array}$ & $\begin{array}{l}- \\
-\end{array}$ & $\begin{array}{l}4.3 \\
0.7\end{array}$ & $\begin{array}{r}18.7 \\
3.3\end{array}$ & - & $\begin{array}{r}31.3 \\
2.1\end{array}$ & $\begin{array}{l}8.2 \\
0.6\end{array}$ & $\begin{array}{l}3.3 \\
-\end{array}$ & $\begin{array}{l}- \\
-\end{array}$ & $\overline{-}$ & - & - & $\begin{array}{l}1.4 \\
0.5\end{array}$ & - & - \\
\hline 9 & $72-81$ & 73.8 & 57.2 & 33.1 & 77.0 & - & 14.3 & 3.6 & - & - & 2.5 & - & 2.0 & 0.6 & - & - & - & - & - & - & - & - \\
\hline 10 & $81-90$ & $\begin{array}{l}86.0 \\
86.6\end{array}$ & $\begin{array}{l}80.4 \\
59.4\end{array}$ & $\begin{array}{l}69.3 \\
36.6\end{array}$ & $\begin{array}{r}7.2 \\
37.8\end{array}$ & $\overline{-}$ & $\overline{61.8}$ & $\begin{array}{r}31.8 \\
0.4\end{array}$ & $\begin{array}{l}- \\
-\end{array}$ & $\begin{array}{l}- \\
-\end{array}$ & $\begin{array}{c}22.4 \\
-\end{array}$ & $\begin{array}{l}3.1 \\
-\end{array}$ & $\begin{array}{c}26.7 \\
-\end{array}$ & $\begin{array}{l}6.3 \\
-\end{array}$ & $\begin{array}{l}2.5 \\
-\end{array}$ & $\begin{array}{l}- \\
-\end{array}$ & $\begin{array}{l}- \\
-\end{array}$ & $\begin{array}{l}- \\
-\end{array}$ & - & - & - & - \\
\hline 11 & $103-112$ & $\begin{array}{l}104.7 \\
105.1\end{array}$ & $\begin{array}{l}66.8 \\
75.3\end{array}$ & $\begin{array}{l}48.2 \\
61.4\end{array}$ & $\begin{array}{r}26.0 \\
6.8\end{array}$ & $\begin{array}{l}- \\
-\end{array}$ & $\begin{array}{r}40.9 \\
1.9\end{array}$ & $\begin{array}{l}23.2 \\
32.2\end{array}$ & $\begin{array}{l}- \\
-\end{array}$ & $\begin{array}{l}- \\
-\end{array}$ & $\begin{array}{r}6.9 \\
33.1\end{array}$ & $\overline{2.1}$ & $\begin{array}{r}2.9 \\
17.2\end{array}$ & $\overline{4.0}$ & $\overline{0.2}$ & $\begin{array}{l}- \\
-\end{array}$ & $\begin{array}{l}- \\
-\end{array}$ & $\begin{array}{l}- \\
-\end{array}$ & - & $\overline{2.4}$ & - & - \\
\hline 12 & $122-131$ & 126.1 & 85.5 & 77.3 & 9.7 & - & - & 24.9 & - & 5.1 & 13.2 & 4.4 & 31.6 & 6.3 & 5.0 & - & - & - & - & - & - & - \\
\hline 20 & $272-281$ & 273.3 & 80.0 & 68.8 & 9.2 & - & - & 30.0 & - & 6.6 & 19.4 & 12.1 & 9.3 & - & 13.4 & - & - & - & - & - & - & - \\
\hline 21 & $291-300$ & $\begin{array}{l}291.7 \\
293.0\end{array}$ & $\begin{array}{l}60.4 \\
70.1\end{array}$ & $\begin{array}{l}38.1 \\
53.3\end{array}$ & $\begin{array}{l}92.7 \\
53.3\end{array}$ & $\begin{array}{l}- \\
-\end{array}$ & $\overline{2.2}$ & $\begin{array}{r}3.2 \\
18.6\end{array}$ & $\begin{array}{l}- \\
-\end{array}$ & $\overline{3.7}$ & $\begin{array}{l}1.3 \\
9.0\end{array}$ & $\overline{2.7}$ & $\begin{array}{l}2.8 \\
7.7\end{array}$ & $\overline{1.6}$ & $\overline{1.3}$ & $\begin{array}{l}- \\
-\end{array}$ & $\begin{array}{l}- \\
-\end{array}$ & - & - & $\begin{array}{l}- \\
-\end{array}$ & - & - \\
\hline 22 & 309-318 & $\begin{array}{l}311.4 \\
315.1 \\
315.3 \\
315.5\end{array}$ & $\begin{array}{l}78.6 \\
82.7 \\
81.0 \\
69.5\end{array}$ & $\begin{array}{l}66.6 \\
73.0 \\
70.2 \\
52.3\end{array}$ & $\begin{array}{r}31.4 \\
5.2 \\
5.6 \\
72.8\end{array}$ & $\begin{array}{l}- \\
- \\
- \\
-\end{array}$ & $\begin{array}{l}19.9 \\
- \\
- \\
-\end{array}$ & $\begin{array}{r}12.7 \\
26.8 \\
23.1 \\
7.2\end{array}$ & $\begin{array}{l}- \\
- \\
- \\
-\end{array}$ & $\begin{array}{l}4.1 \\
6.1 \\
3.5 \\
1.3\end{array}$ & $\begin{array}{r}7.8 \\
15.8 \\
13.6 \\
2.5\end{array}$ & $\begin{array}{l}3.4 \\
1.4 \\
4.1 \\
5.0\end{array}$ & $\begin{array}{r}13.0 \\
29.8 \\
33.4 \\
8.3\end{array}$ & $\begin{array}{l}2.0 \\
8.2 \\
8.8 \\
0.9\end{array}$ & $\begin{array}{l}3.8 \\
6.7 \\
7.8 \\
2.0\end{array}$ & $\begin{array}{l}- \\
- \\
-\end{array}$ & $\begin{array}{l}- \\
- \\
-\end{array}$ & $\begin{array}{l}- \\
- \\
-\end{array}$ & $\begin{array}{l}1.8 \\
- \\
- \\
-\end{array}$ & $\begin{array}{l}- \\
- \\
- \\
-\end{array}$ & $\begin{array}{l}- \\
- \\
-\end{array}$ & $\begin{array}{l}- \\
- \\
-\end{array}$ \\
\hline 23 & $328-337$ & $\begin{array}{l}329.3 \\
334.0\end{array}$ & $\begin{array}{l}72.6 \\
74.9\end{array}$ & $\begin{array}{l}57.2 \\
60.7\end{array}$ & $\begin{array}{l}45.1 \\
10.1\end{array}$ & $\begin{array}{l}- \\
-\end{array}$ & $\begin{array}{l}- \\
-\end{array}$ & $\begin{array}{l}23.1 \\
26.5\end{array}$ & $\begin{array}{l}- \\
-\end{array}$ & $\begin{array}{l}5.0 \\
-\end{array}$ & $\begin{array}{l}11.7 \\
31.1\end{array}$ & $\begin{array}{l}2.2 \\
-\end{array}$ & $\begin{array}{r}9.9 \\
22.6\end{array}$ & $\begin{array}{l}1.3 \\
6.2\end{array}$ & $\begin{array}{l}1.6 \\
1.0\end{array}$ & $\begin{array}{l}- \\
-\end{array}$ & $\begin{array}{l}- \\
-\end{array}$ & $\begin{array}{l}- \\
-\end{array}$ & $\overline{1.1}$ & $\overline{1.3}$ & - & - \\
\hline 24 & $347-356$ & $\begin{array}{l}349.6 \\
355.8\end{array}$ & $\begin{array}{l}77.1 \\
73.3\end{array}$ & $\begin{array}{l}64.3 \\
58.3\end{array}$ & $\begin{array}{l}40.5 \\
24.8\end{array}$ & $\begin{array}{l}- \\
-\end{array}$ & $\begin{array}{r}4.1 \\
10.1\end{array}$ & $\begin{array}{l}13.5 \\
15.3\end{array}$ & - & $\begin{array}{l}2.3 \\
3.1\end{array}$ & $\begin{array}{r}8.7 \\
19.1\end{array}$ & $\begin{array}{l}2.7 \\
-\end{array}$ & $\begin{array}{l}19.0 \\
13.0\end{array}$ & $\begin{array}{l}4.9 \\
3.0\end{array}$ & $\begin{array}{l}4.3 \\
3.4\end{array}$ & $\begin{array}{l}- \\
-\end{array}$ & $\begin{array}{l}- \\
-\end{array}$ & $\overline{7.3}$ & $\overline{0.8}$ & $\begin{array}{l}- \\
-\end{array}$ & - & - \\
\hline 25 & $365-374$ & $\begin{array}{l}365.8 \\
368.2 \\
370.6\end{array}$ & $\begin{array}{l}73.3 \\
67.2 \\
70.3\end{array}$ & $\begin{array}{l}58.2 \\
48.7 \\
53.6\end{array}$ & $\begin{array}{r}51.1 \\
8.4 \\
9.0\end{array}$ & $\begin{array}{l}- \\
- \\
-\end{array}$ & $\begin{array}{l}- \\
- \\
-\end{array}$ & $\begin{array}{r}6.5 \\
40.7 \\
22.5\end{array}$ & $\begin{array}{l}- \\
- \\
-\end{array}$ & $\begin{array}{l}- \\
\overline{5.2}\end{array}$ & $\begin{array}{l}12.4 \\
25.1 \\
27.1\end{array}$ & $\begin{array}{l}- \\
- \\
-\end{array}$ & $\begin{array}{r}5.8 \\
18.9 \\
27.5\end{array}$ & $\begin{array}{l}1.9 \\
5.2 \\
6.3\end{array}$ & $\begin{array}{c}13.6 \\
- \\
1.6\end{array}$ & $\begin{array}{l}- \\
- \\
-\end{array}$ & $\begin{array}{l}- \\
- \\
-\end{array}$ & $\begin{array}{l}8.0 \\
- \\
-\end{array}$ & $\begin{array}{l}- \\
1.6 \\
0.8\end{array}$ & $\begin{array}{l}0.7 \\
- \\
-\end{array}$ & $\begin{array}{l}- \\
- \\
-\end{array}$ & $\begin{array}{l}- \\
- \\
-\end{array}$ \\
\hline 27 & $403-412$ & $\begin{array}{l}407.1 \\
411.9\end{array}$ & $\begin{array}{l}72.2 \\
76.2\end{array}$ & $\begin{array}{l}56.5 \\
62.8\end{array}$ & $\begin{array}{r}3.0 \\
13.8\end{array}$ & $\begin{array}{l}- \\
-\end{array}$ & $\begin{array}{l}- \\
-\end{array}$ & $\begin{array}{l}33.5 \\
31.2\end{array}$ & $\begin{array}{l}- \\
-\end{array}$ & $\begin{array}{l}- \\
-\end{array}$ & $\begin{array}{l}31.5 \\
24.2\end{array}$ & $\begin{array}{l}- \\
-\end{array}$ & $\begin{array}{l}14.7 \\
16.9\end{array}$ & $\begin{array}{l}5.0 \\
5.5\end{array}$ & $\begin{array}{l}3.2 \\
5.2\end{array}$ & - & - & - & $\begin{array}{l}7.8 \\
1.8\end{array}$ & $\begin{array}{l}1.2 \\
1.3\end{array}$ & - & - \\
\hline 28 & $421-430$ & 429.5 & 70.6 & 54.0 & 4.7 & - & - & 27.1 & - & - & 22.3 & - & 35.2 & 8.1 & 1.2 & - & - & - & - & 1.4 & - & - \\
\hline 29 & $440-449$ & 443.9 & 76.2 & 62.6 & - & - & - & 18.7 & - & - & 20.5 & - & 33.2 & 9.6 & 16.1 & - & - & - & 0.6 & 1.4 & $\mathrm{~T}$ & - \\
\hline 30 & $459-468$ & 462.8 & 68.6 & 50.9 & - & - & - & 35.7 & - & - & 28.9 & - & 26.2 & 8.1 & - & - & - & - & - & 1.1 & - & - \\
\hline
\end{tabular}


TABLE 14 - Continued

\begin{tabular}{|c|c|c|c|c|c|c|c|c|c|c|c|c|c|c|c|c|c|c|c|c|c|c|}
\hline Core & $\begin{array}{l}\text { Cored } \\
\text { Interval } \\
\text { Below Sea } \\
\text { Floor }(\mathrm{m})\end{array}$ & $\begin{array}{l}\text { Sample Depth } \\
\text { Below Sea } \\
\text { Floor } \\
\text { (m) }\end{array}$ & 岕 & 宽 & $\frac{\dot{0}}{\pi}$ & $\dot{\grave{g}}$ & 焉 & ةًّ & $\dot{m}$ & $\frac{0}{4}$ & 胫 & 榀 & 党 & 莕 & 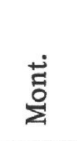 & 岧 & 䞤 & 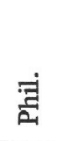 & 莺 & 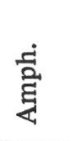 & $\stackrel{\pi}{\vec{b}}$ & गे \\
\hline \multicolumn{23}{|c|}{ Bulk Samples } \\
\hline 31 & $477-486$ & 480.1 & 80.8 & 70.1 & - & - & - & 17.7 & - & 3.8 & 21.0 & - & 38.8 & 6.9 & 10.0 & - & - & - & - & 1.6 & - & - \\
\hline 32 & $496-505$ & 503.7 & 85.7 & 77.7 & - & - & - & 18.6 & - & 14.6 & 11.9 & 3.4 & 16.0 & 3.7 & 31.9 & - & - & - & - & - & - & - \\
\hline 34 & $533-542$ & 533.7 & - & - & 98.0 & - & - & - & - & - & - & - & - & - & - & - & 2.0 & - & - & - & - & - \\
\hline 35 & $542-551$ & 547.7 & 48.9 & 20.2 & 97.4 & - & - & 0.5 & - & - & - & - & - & - & 2.2 & - & - & - & - & - & - & - \\
\hline 36 & $551-560$ & $\begin{array}{l}551.7 \\
555.8\end{array}$ & $\begin{array}{l}56.2 \\
65.8\end{array}$ & $\begin{array}{l}31.6 \\
46.6\end{array}$ & $\begin{array}{l}92.6 \\
78.9\end{array}$ & - & $\begin{array}{l}- \\
-\end{array}$ & $\begin{array}{l}2.1 \\
4.0\end{array}$ & $\overline{2.3}$ & $\begin{array}{l}- \\
-\end{array}$ & $\overline{1.5}$ & - & $\begin{array}{l}1.6 \\
4.6\end{array}$ & - & $\begin{array}{l}2.2 \\
3.3\end{array}$ & $\overline{1.8}$ & $\begin{array}{l}1.4 \\
3.6\end{array}$ & $\begin{array}{l}- \\
-\end{array}$ & $\begin{array}{l}- \\
-\end{array}$ & - & - & $\begin{array}{l}\mathrm{T} \\
\mathrm{T}\end{array}$ \\
\hline 37 & $560-569$ & 564.0 & 61.5 & 39.8 & 78.2 & - & - & 4.0 & - & - & 1.5 & - & 5.2 & - & 8.7 & - & 2.4 & - & - & - & - & $\mathrm{T}$ \\
\hline 38 & $569-578$ & 571.3 & 63.9 & 43.5 & 82.4 & - & - & 3.7 & 3.7 & - & 1.0 & - & 3.5 & - & 4.5 & 1.2 & - & - & - & - & - & $\mathrm{T}$ \\
\hline 39 & $578-587$ & 579.8 & - & - & 81.7 & - & - & 2.4 & 9.0 & - & - & - & 2.2 & - & 1.5 & 3.2 & - & - & - & - & - & $\mathrm{T}$ \\
\hline 40 & $587-596$ & 590.4 & - & - & 63.0 & - & - & 3.7 & 18.2 & - & 1.1 & - & 4.1 & - & 7.4 & 2.5 & - & - & - & - & - & $\mathrm{T}$ \\
\hline 41 & 596-605 & 597.5 & - & - & 73.9 & - & - & 4.6 & 12.6 & - & 1.8 & - & 2.4 & - & 2.0 & 2.8 & - & - & - & - & - & $\mathrm{T}$ \\
\hline 43 & $614-623$ & 619.4 & 57.6 & 33.8 & 65.7 & 4.7 & - & 23.0 & - & - & - & - & 3.0 & - & 3.6 & - & - & - & - & - & - & $\mathrm{T}$ \\
\hline 44 & $623-632$ & 625.5 & - & - & 65.7 & - & - & 26.6 & - & - & 0.7 & - & 4.4 & - & 2.7 & - & - & - & - & - & - & - \\
\hline 45 & $632-641$ & 634.5 & 55.9 & 31.2 & 55.4 & - & - & 33.2 & - & - & - & - & 5.7 & - & - & 5.7 & - & - & - & - & - & $\mathrm{T}$ \\
\hline 46 & $648-657$ & 653.1 & 57.2 & 33.1 & 67.7 & - & - & 22.7 & - & - & - & - & 4.6 & 0.7 & 4.3 & - & - & - & - & - & - & - \\
\hline 47 & $665-674$ & 668.3 & 61.6 & 40.0 & 54.4 & - & - & 34.2 & - & - & 0.8 & - & 5.4 & 1.5 & 3.6 & - & - & - & - & - & - & $\mathrm{T}$ \\
\hline 48 & $678-687$ & 680.0 & 59.0 & 35.9 & 61.3 & - & - & 28.4 & - & - & 1.1 & - & 5.3 & 1.5 & 2.5 & - & - & - & - & - & - & $\mathrm{T}$ \\
\hline 49 & $692-701$ & 694.3 & 59.0 & 35.9 & 62.6 & - & - & 29.2 & - & - & 1.2 & - & 4.3 & 1.7 & 1.1 & - & - & - & - & - & - & $\mathrm{T}$ \\
\hline 50 & $702-711$ & 707.8 & 62.9 & 42.0 & 40.6 & - & - & 41.9 & - & - & 1.3 & - & 12.0 & 4.3 & - & - & - & - & - & - & - & - \\
\hline
\end{tabular}




\begin{tabular}{|c|c|c|c|c|c|c|c|c|c|c|c|c|c|c|c|c|c|c|c|}
\hline Core & $\begin{array}{l}\text { Cored } \\
\text { Interval } \\
\text { Below Sea } \\
\text { Floor (m) } \\
\end{array}$ & $\begin{array}{l}\text { Sample Depth } \\
\text { Below Sea } \\
\text { Floor } \\
\text { (m) }\end{array}$ & 幽 & 窨 & 岕 & 离 & 这 & 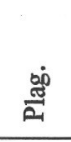 & $\begin{array}{l}\ddot{\Xi} \\
\ddot{\pi}\end{array}$ & 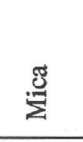 & 递 & $\stackrel{\stackrel{\Xi}{\Xi}}{\Sigma}$ & 葛 & 宊 & 竎 & 总 & 远 & 客 & "ే \\
\hline \multicolumn{20}{|c|}{$2-20 \mu$ Fraction } \\
\hline 1 & $0-9$ & $\begin{array}{l}0.03 \\
4.7 \\
8.8\end{array}$ & $\begin{array}{l}78.0 \\
70.0 \\
72.8\end{array}$ & $\begin{array}{l}65.7 \\
53.1 \\
57.5\end{array}$ & $\begin{array}{r}37.0 \\
37.5 \\
1.2\end{array}$ & $\begin{array}{l}- \\
- \\
-\end{array}$ & $\begin{array}{l}- \\
- \\
-\end{array}$ & $\begin{array}{l}27.9 \\
23.6 \\
98.7\end{array}$ & $\begin{array}{l}2.0 \\
- \\
-\end{array}$ & $\begin{array}{c}23.9 \\
30.1 \\
-\end{array}$ & $\begin{array}{l}7.5 \\
7.6 \\
-\end{array}$ & $\begin{array}{l}- \\
- \\
-\end{array}$ & $\begin{array}{l}- \\
- \\
-\end{array}$ & $\begin{array}{l}- \\
- \\
-\end{array}$ & $\begin{array}{l}- \\
- \\
-\end{array}$ & $\begin{array}{l}- \\
- \\
-\end{array}$ & $\begin{array}{l}- \\
- \\
-\end{array}$ & $\begin{array}{l}1.7 \\
1.2 \\
0.1\end{array}$ & $\begin{array}{l}- \\
- \\
-\end{array}$ \\
\hline 2 & $9-18$ & $\begin{array}{r}9.4 \\
12.8 \\
16.8 \\
17.0\end{array}$ & $\begin{array}{l}71.3 \\
70.7 \\
72.8 \\
77.9\end{array}$ & $\begin{array}{l}55.2 \\
54.1 \\
57.5 \\
65.5\end{array}$ & $\begin{array}{l}38.8 \\
37.4 \\
32.0 \\
34.0\end{array}$ & $\begin{array}{l}- \\
- \\
- \\
-\end{array}$ & $\begin{array}{l}- \\
5.1 \\
5.2 \\
3.2\end{array}$ & $\begin{array}{l}28.0 \\
31.4 \\
27.4 \\
23.9\end{array}$ & $\begin{array}{l}- \\
- \\
- \\
-\end{array}$ & $\begin{array}{l}24.9 \\
17.6 \\
24.6 \\
29.5\end{array}$ & $\begin{array}{l}5.4 \\
5.3 \\
7.7 \\
7.6\end{array}$ & $\begin{array}{l}- \\
- \\
- \\
-\end{array}$ & $\begin{array}{l}- \\
- \\
-\end{array}$ & $\begin{array}{l}- \\
- \\
- \\
-\end{array}$ & $\begin{array}{l}- \\
- \\
-\end{array}$ & $\begin{array}{l}0.9 \\
- \\
0.5 \\
-\end{array}$ & $\begin{array}{l}- \\
- \\
-\end{array}$ & $\begin{array}{l}2.1 \\
3.2 \\
2.5 \\
1.7\end{array}$ & $\begin{array}{l}- \\
- \\
- \\
-\end{array}$ \\
\hline 3 & $18-27$ & $\begin{array}{l}21.1 \\
23.2\end{array}$ & $\begin{array}{l}71.9 \\
71.7\end{array}$ & $\begin{array}{l}56.0 \\
55.7\end{array}$ & $\begin{array}{l}44.6 \\
58.9\end{array}$ & - & $\overline{4.8}$ & $\begin{array}{l}24.9 \\
19.5\end{array}$ & - & $\begin{array}{l}21.8 \\
11.8\end{array}$ & $\begin{array}{l}6.2 \\
1.9\end{array}$ & $\begin{array}{l}- \\
-\end{array}$ & - & - & - & $\begin{array}{l}1.1 \\
2.1\end{array}$ & - & $\begin{array}{l}1.3 \\
1.1\end{array}$ & - \\
\hline 6 & $45-54$ & 49.4 & 65.2 & 45.6 & 53.8 & - & 5.5 & 17.0 & 3.5 & 17.5 & 1.7 & - & - & - & - & 1.0 & - & - & - \\
\hline 8 & $63-72$ & $\begin{array}{l}69.7 \\
70.2\end{array}$ & $\begin{array}{l}66.0 \\
68.2\end{array}$ & $\begin{array}{l}46.9 \\
50.3\end{array}$ & $\begin{array}{l}28.4 \\
49.5\end{array}$ & $\begin{array}{l}- \\
-\end{array}$ & $\begin{array}{l}4.5 \\
6.7\end{array}$ & $\begin{array}{l}20.3 \\
20.3\end{array}$ & $\begin{array}{l}- \\
-\end{array}$ & $\begin{array}{l}35.8 \\
16.5\end{array}$ & $\begin{array}{l}9.7 \\
3.5\end{array}$ & - & - & - & $\begin{array}{l}- \\
-\end{array}$ & $\overline{3.5}$ & $\begin{array}{l}- \\
-\end{array}$ & $\begin{array}{l}1.3 \\
-\end{array}$ & - \\
\hline 9 & $72-81$ & 73.8 & 75.9 & 62.4 & 35.5 & - & 3.3 & 29.7 & - & 22.2 & 8.0 & - & - & - & - & - & - & 1.3 & - \\
\hline 10 & $81-90$ & 86.0 & 68.5 & 50.8 & 32.0 & - & 2.7 & 25.4 & - & 29.2 & 8.1 & - & - & - & - & 1.1 & - & 1.6 & - \\
\hline 11 & $103-112$ & $\begin{array}{l}104.7 \\
105.1\end{array}$ & $\begin{array}{l}61.4 \\
68.9\end{array}$ & $\begin{array}{l}39.7 \\
51.4\end{array}$ & $\begin{array}{l}56.2 \\
29.7\end{array}$ & $\begin{array}{l}- \\
-\end{array}$ & $\begin{array}{l}8.7 \\
5.2\end{array}$ & $\begin{array}{l}23.6 \\
30.2\end{array}$ & $\begin{array}{l}- \\
-\end{array}$ & $\begin{array}{r}6.9 \\
23.3\end{array}$ & $\begin{array}{l}0.7 \\
6.8\end{array}$ & $\begin{array}{l}- \\
-\end{array}$ & - & $\begin{array}{l}- \\
-\end{array}$ & $\begin{array}{l}- \\
-\end{array}$ & $\begin{array}{l}2.0 \\
1.3\end{array}$ & - & $\begin{array}{l}2.0 \\
3.4\end{array}$ & - \\
\hline 12 & $122-131$ & 126.1 & 70.2 & 53.4 & 33.6 & - & 5.4 & 21.9 & 3.4 & 29.4 & 6.2 & - & - & - & - & - & - & - & - \\
\hline 20 & $272-281$ & 273.3 & 67.3 & 48.9 & 28.5 & - & 6.6 & 19.6 & - & 35.4 & 9.5 & - & - & - & - & 0.5 & - & - & - \\
\hline 21 & $291-300$ & $\begin{array}{l}291.7 \\
293.0\end{array}$ & $\begin{array}{l}73.0 \\
67.6\end{array}$ & $\begin{array}{l}57.8 \\
49.4\end{array}$ & $\begin{array}{l}37.8 \\
25.6\end{array}$ & - & $\begin{array}{r}11.4 \\
8.1\end{array}$ & $\begin{array}{l}24.9 \\
22.0\end{array}$ & $\begin{array}{l}- \\
-\end{array}$ & $\begin{array}{l}20.1 \\
31.4\end{array}$ & $\begin{array}{l}5.7 \\
8.2\end{array}$ & $\overline{4.8}$ & - & - & $\begin{array}{l}- \\
-\end{array}$ & $\begin{array}{l}- \\
-\end{array}$ & - & $\begin{array}{l}- \\
-\end{array}$ & - \\
\hline 22 & $309-318$ & $\begin{array}{l}311.4 \\
315.1 \\
315.3 \\
315.5\end{array}$ & $\begin{array}{l}68.1 \\
59.6 \\
66.9 \\
66.7\end{array}$ & $\begin{array}{l}50.2 \\
36.8 \\
48.3 \\
48.0\end{array}$ & $\begin{array}{l}30.8 \\
52.5 \\
26.0 \\
45.1\end{array}$ & $\begin{array}{l}- \\
- \\
- \\
-\end{array}$ & $\begin{array}{l}4.2 \\
9.3 \\
6.0 \\
7.1\end{array}$ & $\begin{array}{l}27.0 \\
22.3 \\
18.3 \\
17.1\end{array}$ & $\begin{array}{l}- \\
\overline{-} \\
\overline{2.4}\end{array}$ & $\begin{array}{l}23.2 \\
10.8 \\
38.4 \\
24.4\end{array}$ & $\begin{array}{r}7.5 \\
1.4 \\
11.2 \\
3.9\end{array}$ & $\begin{array}{l}- \\
- \\
- \\
-\end{array}$ & $\begin{array}{l}- \\
- \\
-\end{array}$ & $\begin{array}{l}- \\
\overline{-} \\
-\end{array}$ & $\begin{array}{l}- \\
- \\
- \\
-\end{array}$ & $\begin{array}{l}6.1 \\
2.6 \\
- \\
-\end{array}$ & $\begin{array}{l}- \\
- \\
-\end{array}$ & $\begin{array}{l}1.2 \\
0.9 \\
- \\
-\end{array}$ & $\begin{array}{l}- \\
- \\
-\end{array}$ \\
\hline 23 & $328-337$ & $\begin{array}{l}329.3 \\
334.0\end{array}$ & $\begin{array}{l}57.7 \\
68.4\end{array}$ & $\begin{array}{l}34.0 \\
50.6\end{array}$ & $\begin{array}{l}46.7 \\
26.6\end{array}$ & $\begin{array}{l}- \\
-\end{array}$ & $\begin{array}{l}9.4 \\
-\end{array}$ & $\begin{array}{l}24.8 \\
34.1\end{array}$ & $\begin{array}{l}2.6 \\
-\end{array}$ & $\begin{array}{l}13.3 \\
24.9\end{array}$ & $\begin{array}{r}0.6 \\
11.1\end{array}$ & $\begin{array}{l}- \\
-\end{array}$ & - & - & - & $\begin{array}{l}0.8 \\
1.9\end{array}$ & $\begin{array}{l}- \\
-\end{array}$ & $\begin{array}{l}1.7 \\
1.4\end{array}$ & $\overline{\mathrm{T}}$ \\
\hline 24 & $347-356$ & $\begin{array}{l}349.6 \\
355.8\end{array}$ & $\begin{array}{l}68.2 \\
67.3\end{array}$ & $\begin{array}{l}50.3 \\
48.9\end{array}$ & $\begin{array}{l}27.1 \\
16.3\end{array}$ & $\begin{array}{l}- \\
-\end{array}$ & $\begin{array}{l}5.7 \\
4.4\end{array}$ & $\begin{array}{l}24.5 \\
18.9\end{array}$ & $\begin{array}{l}- \\
-\end{array}$ & $\begin{array}{l}27.8 \\
11.8\end{array}$ & $\begin{array}{r}9.0 \\
38.6\end{array}$ & $\begin{array}{l}6.0 \\
-\end{array}$ & - & $\overline{0.6}$ & $\overline{8.3}$ & - & $\begin{array}{l}- \\
-\end{array}$ & $\overline{1.1}$ & - \\
\hline 25 & $365-374$ & $\begin{array}{l}368.2 \\
370.6\end{array}$ & $\begin{array}{l}62.9 \\
63.5\end{array}$ & $\begin{array}{l}42.0 \\
43.0\end{array}$ & $\begin{array}{l}31.7 \\
26.2\end{array}$ & $\begin{array}{l}- \\
-\end{array}$ & $\overline{8.4}$ & $\begin{array}{l}21.7 \\
29.2\end{array}$ & $\begin{array}{l}- \\
-\end{array}$ & $\begin{array}{l}33.6 \\
25.9\end{array}$ & $\begin{array}{r}11.6 \\
7.0\end{array}$ & $\begin{array}{l}- \\
-\end{array}$ & - & - & - & $\begin{array}{l}1.4 \\
0.9\end{array}$ & $\begin{array}{l}- \\
-\end{array}$ & $\overline{2.5}$ & $\begin{array}{l}- \\
-\end{array}$ \\
\hline 27 & $403-412$ & $\begin{array}{l}407.1 \\
411.9\end{array}$ & $\begin{array}{l}69.3 \\
71.3\end{array}$ & $\begin{array}{l}52.0 \\
55.2\end{array}$ & $\begin{array}{l}29.2 \\
33.2\end{array}$ & $\begin{array}{l}- \\
-\end{array}$ & $\begin{array}{l}- \\
-\end{array}$ & $\begin{array}{l}21.5 \\
25.5\end{array}$ & $\overline{2.4}$ & $\begin{array}{l}18.3 \\
17.2\end{array}$ & $\begin{array}{l}6.6 \\
5.2\end{array}$ & $\begin{array}{r}8.0 \\
11.9\end{array}$ & - & - & - & $\begin{array}{r}14.5 \\
3.4\end{array}$ & $\begin{array}{l}- \\
-\end{array}$ & $\begin{array}{l}2.0 \\
1.2\end{array}$ & - \\
\hline 28 & $421-430$ & 429.5 & 59.4 & 36.3 & 22.6 & - & - & 21.1 & - & 39.1 & 13.4 & - & - & - & - & 0.8 & - & 3.1 & - \\
\hline 29 & $440-449$ & 443.9 & 62.2 & 41.0 & 19.9 & - & - & 20.9 & - & 42.4 & 13.8 & - & - & - & - & 0.6 & - & 2.4 & $\mathrm{~T}$ \\
\hline 30 & $459-468$ & 462.8 & 58.0 & 34.3 & 25.3 & - & - & 23.3 & - & 36.2 & 13.8 & - & - & - & - & - & - & 2.5 & - \\
\hline 31 & $477-486$ & 480.1 & 66.9 & 48.2 & 24.0 & - & 5.6 & 27.5 & - & 31.5 & 10.0 & - & - & - & - & - & - & 1.4 & - \\
\hline 32 & $496-505$ & 503.7 & 75.6 & 61.9 & 30.4 & - & 26.0 & 20.9 & 3.0 & 17.2 & 2.4 & - & - & - & - & - & - & - & - \\
\hline
\end{tabular}




\begin{tabular}{|c|c|c|c|c|c|c|c|c|c|c|c|c|c|c|c|c|c|c|c|}
\hline 36 & $551-560$ & $\begin{array}{l}551.7 \\
555.8\end{array}$ & $\begin{array}{l}66.9 \\
67.3\end{array}$ & $\begin{array}{l}48.3 \\
49.0\end{array}$ & $\begin{array}{l}34.4 \\
28.2\end{array}$ & $\overline{3} .0$ & - & $\begin{array}{l}14.3 \\
12.4\end{array}$ & $\begin{array}{l}- \\
-\end{array}$ & $\begin{array}{l}20.4 \\
28.7\end{array}$ & $\begin{array}{l}- \\
-\end{array}$ & $\begin{array}{l}- \\
-\end{array}$ & $\overline{2.2}$ & $\begin{array}{l}30.9 \\
25.6\end{array}$ & $\begin{array}{l}- \\
-\end{array}$ & $\begin{array}{l}- \\
-\end{array}$ & $\begin{array}{l}- \\
-\end{array}$ & $\begin{array}{l}- \\
-\end{array}$ & - \\
\hline 37 & $560-569$ & 564.0 & 66.7 & 47.9 & 36.9 & - & - & 16.4 & - & 21.7 & - & - & - & 25.1 & - & - & - & - & - \\
\hline 39 & $578-587$ & 579.8 & 83.4 & 74.1 & 17.8 & 50.5 & - & 8.5 & - & 12.5 & 0.8 & - & 9.1 & 0.8 & - & - & - & - & - \\
\hline 40 & $587-596$ & 590.4 & 79.1 & 67.4 & 16.0 & 47.4 & - & 6.5 & - & 15.0 & 1.0 & 10.3 & 3.8 & - & - & - & - & - & - \\
\hline 41 & $596-605$ & 597.5 & 77.4 & 64.8 & 26.9 & 39.5 & 4.2 & 8.5 & - & 16.2 & 0.8 & - & 3.9 & - & - & - & - & - & - \\
\hline 42 & $605-614$ & 605.6 & 76.1 & 62.6 & 32.2 & 27.9 & 4.3 & 8.3 & - & 23.1 & - & - & 4.3 & - & - & - & - & - & $\mathbf{T}$ \\
\hline 43 & $614-623$ & 619.4 & 62.4 & 41.2 & 65.3 & - & 6.7 & 6.4 & - & 18.6 & 1.5 & - & - & - & - & - & 1.5 & - & - \\
\hline 44 & $623-632$ & 625.5 & 60.4 & 38.1 & 63.1 & - & 6.8 & 5.0 & - & 21.5 & - & - & - & - & - & 1.2 & 2.3 & - & - \\
\hline 45 & $632-641$ & 634.5 & 57.7 & 34.0 & 71.1 & - & 5.1 & 4.9 & - & 16.8 & 1.1 & - & - & - & - & 1.0 & - & - & - \\
\hline 46 & $648-657$ & 653.1 & 63.1 & 42.3 & 66.0 & - & 6.4 & 5.8 & - & 18.3 & 1.0 & - & - & - & - & - & 2.6 & - & - \\
\hline 47 & $665-674$ & 668.3 & 57.0 & 32.8 & 68.2 & - & - & 11.7 & - & 15.7 & 2.9 & - & - & - & - & - & 1.5 & - & - \\
\hline 49 & $692-701$ & 694.3 & 58.2 & 34.7 & 84.7 & - & - & 10.5 & - & - & 3.0 & - & - & - & - & - & 1.9 & - & - \\
\hline 50 & $702-711$ & 707.8 & 51.5 & 24.2 & 68.4 & - & - & 10.4 & - & 17.2 & 4.1 & - & - & - & - & - & - & - & - \\
\hline Core & $\begin{array}{l}\text { Cored } \\
\text { Interval } \\
\text { Below Sea } \\
\text { Floor (m) }\end{array}$ & $\begin{array}{l}\text { Sample Depth } \\
\text { Below Sea } \\
\text { Floor } \\
\text { (m) }\end{array}$ & 㟧 & 宫 & 苞 & $\dot{\mathscr{J}}$ & 离 & 离 & تृ & 氖 & 远 & 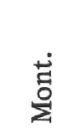 & 怘 & 竎 & :ं & $\underset{⿱ 乛 龰}{\stackrel{5}{*}}$ & 莕 & $\stackrel{5}{\vec{b}}$ & in \\
\hline
\end{tabular}

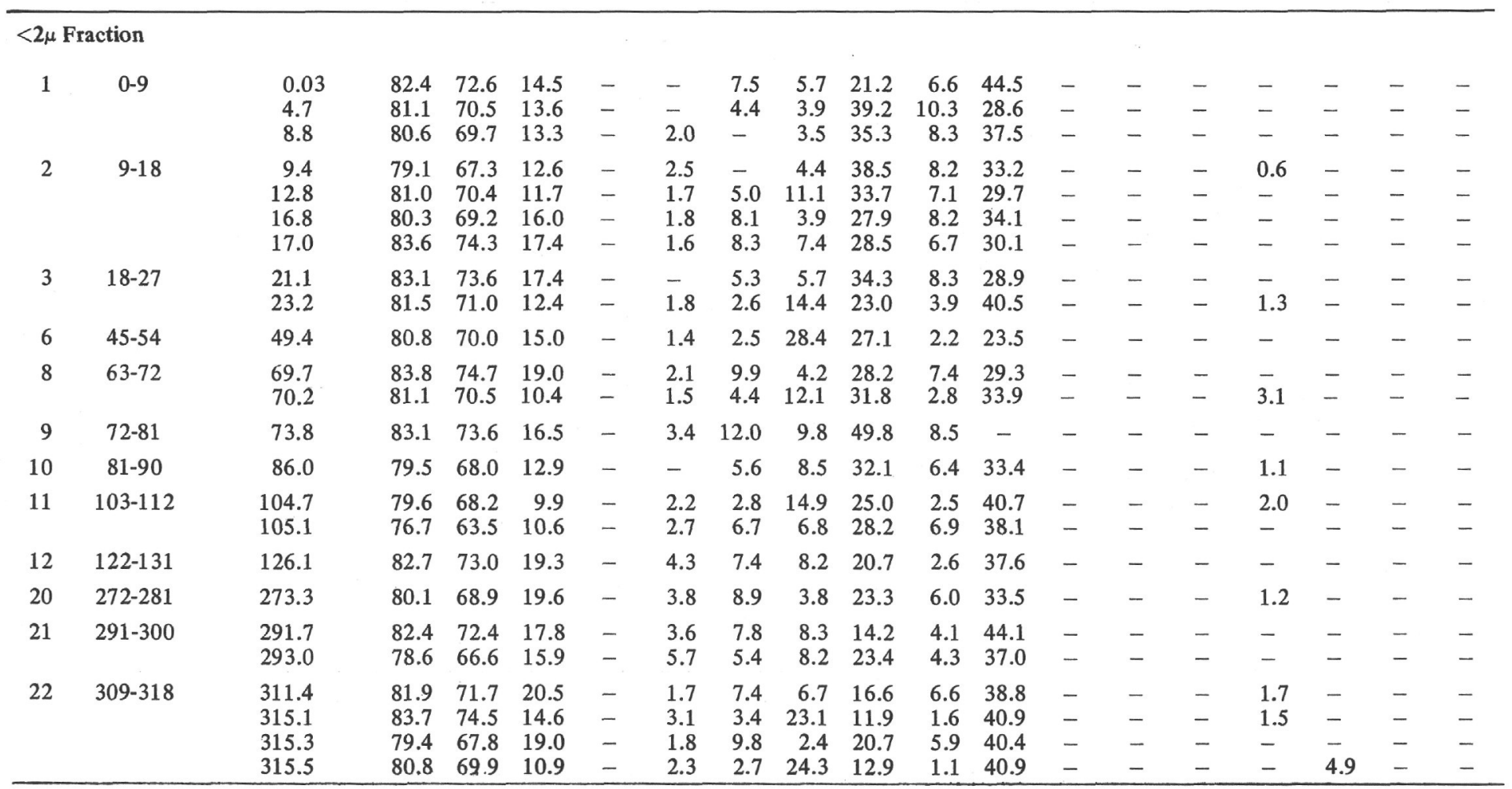




\begin{tabular}{|c|c|c|c|c|c|c|c|c|c|c|c|c|c|c|c|c|c|c|c|}
\hline Core & $\begin{array}{c}\text { Cored } \\
\text { Interval } \\
\text { Below Sea } \\
\text { Floor (m) }\end{array}$ & $\begin{array}{l}\text { Sample Depth } \\
\text { Below Sea } \\
\text { Floor } \\
\text { (m) }\end{array}$ & 名 & 范 & 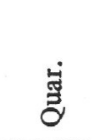 & 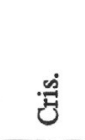 & 它 & 苞 & 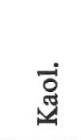 & 尝 & 럼 & 苛 & 苞 & घं & 齐 & 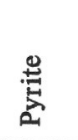 & 离 & $\overrightarrow{5}$ & in \\
\hline 23 & $328-337$ & $\begin{array}{l}329.3 \\
334.0\end{array}$ & $\begin{array}{l}79.9 \\
71.1\end{array}$ & $\begin{array}{l}68.5 \\
54.9\end{array}$ & $\begin{array}{r}12.0 \\
9.2\end{array}$ & - & $\begin{array}{l}2.6 \\
6.8\end{array}$ & $\begin{array}{l}3.0 \\
5.1\end{array}$ & $\begin{array}{r}24.9 \\
6.1\end{array}$ & $\begin{array}{l}10.9 \\
23.8\end{array}$ & $\begin{array}{l}2.6 \\
3.9\end{array}$ & $\begin{array}{l}44.0 \\
45.0\end{array}$ & - & - & - & $\overline{-}$ & $=$ & - & - \\
\hline 24 & $347-356$ & $\begin{array}{l}349.6 \\
355.8\end{array}$ & $\begin{array}{l}81.2 \\
81.0\end{array}$ & $\begin{array}{l}70.7 \\
70.3\end{array}$ & $\begin{array}{r}17.7 \\
6.8\end{array}$ & - & $\begin{array}{l}3.2 \\
2.9\end{array}$ & $\begin{array}{l}9.8 \\
5.4\end{array}$ & 2.7 & $\begin{array}{r}22.9 \\
7.0\end{array}$ & $\begin{array}{l}6.2 \\
3.4\end{array}$ & $\begin{array}{l}37.4 \\
64.6\end{array}$ & - & - & $\overline{9.9}$ & - & $\overline{-}$ & - & - \\
\hline 25 & $365-374$ & $\begin{array}{l}365.8 \\
368.2 \\
370.6\end{array}$ & $\begin{array}{l}76.0 \\
71.7 \\
84.7\end{array}$ & $\begin{array}{l}62.4 \\
55.8 \\
76.1\end{array}$ & $\begin{array}{l}1.7 \\
9.7 \\
7.6\end{array}$ & $\begin{array}{l}- \\
- \\
-\end{array}$ & $\begin{array}{l}5.5 \\
2.5 \\
2.3\end{array}$ & $\begin{array}{l}5.9 \\
3.6 \\
4.6\end{array}$ & $\begin{array}{l}- \\
1.9 \\
-\end{array}$ & $\begin{array}{r}5.7 \\
42.7 \\
23.5\end{array}$ & $\begin{array}{l}2.3 \\
9.6 \\
6.8\end{array}$ & $\begin{array}{l}71.2 \\
29.2 \\
53.4\end{array}$ & $\begin{array}{l}- \\
- \\
-\end{array}$ & $\begin{array}{l}- \\
\overline{0} .9\end{array}$ & $\begin{array}{l}7.8 \\
- \\
-\end{array}$ & $\begin{array}{l}- \\
0.9 \\
0.9\end{array}$ & $\begin{array}{l}- \\
-\end{array}$ & $\begin{array}{l}- \\
- \\
-\end{array}$ & $\begin{array}{l}- \\
- \\
-\end{array}$ \\
\hline 27 & $403-412$ & $\begin{array}{l}407.1 \\
411.9\end{array}$ & $\begin{array}{l}74.0 \\
84.2\end{array}$ & $\begin{array}{l}59.4 \\
75.3\end{array}$ & $\begin{array}{l}13.6 \\
11.3\end{array}$ & - & - & $\begin{array}{l}5.3 \\
3.6\end{array}$ & $\overline{8.4}$ & $\begin{array}{l}18.7 \\
11.0\end{array}$ & $\begin{array}{l}6.3 \\
1.8\end{array}$ & $\begin{array}{l}54.2 \\
62.3\end{array}$ & - & - & - & $\begin{array}{l}1.8 \\
1.6\end{array}$ & $=$ & - & $=$ \\
\hline 28 & $421-430$ & 429.5 & 73.9 & 59.2 & 11.6 & - & 4.8 & 7.6 & - & 40.8 & 11.5 & 22.7 & - & - & - & 1.0 & - & - & - \\
\hline 29 & $440-449$ & 443.9 & 84.1 & 75.2 & 12.3 & - & - & 11.3 & - & 26.2 & - & 50.2 & - & - & - & - & - & $\mathrm{T}$ & - \\
\hline 30 & $459-468$ & 462.8 & 76.2 & 62.8 & 14.0 & - & - & 12.6 & - & 30.9 & 10.8 & 31.8 & - & - & - & - & - & - & - \\
\hline 31 & $477-486$ & 480.1 & 81.5 & 71.1 & 15.3 & - & 3.3 & 16.3 & - & 28.3 & 9.7 & 27.1 & - & - & - & - & - & - & - \\
\hline 32 & $296-505$ & 503.7 & 84.6 & 75.9 & 9.5 & - & 7.3 & 6.5 & 9.1 & 14.7 & 3.0 & 49.9 & - & - & - & - & - & - & - \\
\hline 34 & $533-542$ & 533.7 & 82.3 & 72.4 & 6.0 & - & - & 2.9 & - & 4.2 & 1.2 & 74.2 & - & 11.5 & - & - & - & - & - \\
\hline 35 & $542-551$ & 547.7 & 72.9 & 57.6 & 3.2 & - & - & 2.8 & - & 2.7 & - & 90.3 & - & 0.9 & - & - & - & - & - \\
\hline 36 & $551-560$ & $\begin{array}{l}551.7 \\
555.8\end{array}$ & $\begin{array}{l}79.7 \\
84.5\end{array}$ & $\begin{array}{l}68.2 \\
75.7\end{array}$ & $\begin{array}{l}9.1 \\
7.7\end{array}$ & $\overline{28.6}$ & $\overline{-}$ & $\begin{array}{l}1.1 \\
1.8\end{array}$ & - & $\begin{array}{l}11.5 \\
10.6\end{array}$ & - & $\begin{array}{l}75.4 \\
41.7\end{array}$ & $\overline{6.5}$ & $\begin{array}{l}2.9 \\
3.2\end{array}$ & - & - & $\overline{-}$ & $\overline{-}$ & $\overline{-}$ \\
\hline 37 & $560-569$ & 564.0 & 76.5 & 63.3 & 9.6 & - & - & 0.7 & - & 17.0 & 0.8 & 68.8 & - & 3.1 & - & - & - & - & - \\
\hline 38 & 569-578 & 571.3 & 81.2 & 70.6 & 6.8 & 34.7 & - & 2.0 & - & 8.9 & 0.8 & 43.0 & 2.3 & 1.6 & - & - & - & - & - \\
\hline 39 & 578-587 & 579.8 & 85.4 & 77.2 & 3.3 & 66.0 & - & 1.4 & - & 2.4 & - & 16.6 & 10.2 & - & - & - & - & - & - \\
\hline 40 & $587-596$ & 590.4 & 81.6 & 71.3 & 4.1 & 56.3 & - & 1.4 & - & 1.7 & - & 26.6 & 9.8 & - & - & - & - & - & - \\
\hline 41 & $596-605$ & 597.5 & 82.7 & 73.0 & 8.9 & 62.2 & - & 1.5 & - & 2.3 & - & 16.9 & 8.2 & - & - & - & - & - & - \\
\hline 42 & $605-614$ & 605.6 & 81.2 & 70.6 & 12.8 & 55.8 & - & 0.9 & - & 3.6 & - & 20.1 & 6.7 & - & - & - & - & $\mathrm{T}$ & - \\
\hline 43 & $614-623$ & 619.4 & 63.9 & 43.7 & 77.7 & - & - & - & - & 3.1 & - & 19.2 & - & - & - & - & - & - & - \\
\hline 44 & $623-632$ & 625.5 & 61.4 & 39.8 & 75.6 & - & - & - & - & 5.1 & - & 19.3 & - & - & - & - & - & - & - \\
\hline 45 & $632-641$ & 634.5 & 57.3 & 33.2 & 76.9 & - & - & - & - & 6.7 & - & 16.5 & - & - & - & - & - & - & - \\
\hline 46 & $648-657$ & 653.1 & 60.1 & 37.6 & 63.7 & - & - & - & - & 8.7 & 2.2 & 25.4 & - & - & - & - & - & - & $\mathbf{P}$ \\
\hline 47 & $665-674$ & 668.3 & 66.4 & 47.6 & 74.8 & - & - & - & - & 7.9 & 3.2 & 14.0 & - & - & - & - & - & - & - \\
\hline 48 & $678-687$ & 680.0 & 64.3 & 44.2 & 72.0 & - & - & - & - & 10.3 & 3.6 & 14.1 & - & - & - & - & - & - & - \\
\hline 49 & $692-701$ & 694.3 & 71.9 & 56.1 & 63.6 & - & - & 0.8 & - & 15.5 & 7.0 & 13.1 & - & - & - & - & - & - & - \\
\hline 50 & $702-711$ & 707.8 & 63.8 & 43.5 & 77.4 & - & - & 1.2 & - & 12.2 & 9.2 & - & - & - & - & - & - & - & - \\
\hline
\end{tabular}

${ }^{a}$ Peaks at $12.1 \AA$

Peaks at $3.14 \AA$. 